\title{
Existence of extensions of semilattices of groups by groups, cohomology, and crossed modules for inverse semigroups
}

\author{
Thais Mayumi Batista Makuta
}

\author{
TESE APRESENTADA \\ $\mathrm{AO}$, \\ InSTITUTO DE MATEMÁtica E Estatística \\ DA \\ Universidade De SÃo PaUlo \\ PARA \\ OBTENÇẪO DO TÍTULO \\ Doutora EM CIÊNCIAS
}

Programa: Matemática

Orientador: Prof. Dr. Mikhailo Dokuchaev (USP)

Coorientador: Prof. Dr. Mykola Khrypchenko (UFSC)

O presente trabalho foi realizado com apoio da Coordenação de Aperfeiçoamento de Pessoal de Nível Superior - Brasil (CAPES) - Código de Financiamento 001 
Existence of extensions of semilattices of groups by groups, cohomology, and crossed modules for inverse semigroups

Esta é a versão original da tese elaborada pela candidata Thais Mayumi Batista Makuta, tal como submetida à Comissão Julgadora. 


\section{Resumo}

Makuta, T. M. B. Existência de extensões de semirreticulados de grupos por grupos, cohomologia, e módulos cruzados para semigrupos inversos. Tese (Doutorado) - Instituto de Matemática e Estatística, Universidade de São Paulo, São Paulo, 2019.

Introduz-se o conceito de um núcleo abstrato parcial associado a um $\operatorname{par}(G, A)$, em que $G$ é um grupo e $A$ é um semirreticulado de grupos, e relaciona-se o grupo de cohomologia parcial $H^{3}(G, C(A))$ às obstruções a existência de extensões admissíveis de $A$ por $G$ que realizam o núcleo abstrato dado. Também, se tais extensões existem, mostra-se que elas são classificadas por $H^{2}(G, C(A))$. Define-se a noção de módulo cruzado sobre um semigrupo inverso e constrói-se uma sequência de quatro termos correspondente. A cada classe de equivalência de tais sequências relaciona-se um elemento do terceiro grupo das cohomologias que perservam ordem, que no caso de semirreticulado de grupos resulta numa bijeção.

Palavras-chave: ação parcial, núcleo abstrato, cohomologia de ação parcial, semigrupos inversos, cohomologia de semigrupos inversos, extensões, semirreticulados de grupos. 


\section{Abstract}

Makuta, T. M. B. Existence of extensions of semilattices of groups by groups, cohomology, and crossed modules for inverse semigroups. Tese (Doutorado) - Instituto de Matemática e Estatística, Universidade de São Paulo, São Paulo, 2019.

We introduce the concept of a partial abstract kernel associated to a pair $(G, A)$, where $G$ is a group and $A$ is a semilattice of groups, and relate the partial cohomology group $H^{3}(G, C(A))$ with the obstructions to the existence of admissible extensions of $A$ by $G$ which realize the given abstract kernel. Also, if such extensions exist, we show that they are classified by $H^{2}(G, C(A))$. We define the notion of a crossed module over inverse semigroups and construct a corresponding 4-term sequence. To each equivalence class of such sequences we relate an element of the third order-preserving inverse semigroup cohomology, so that we have a bijection in the case of a semilattice of groups.

Keywords: partial action, abstract kernel, partial group cohomology, inverse semigroups, inverse semigroup cohomology, extensions, semilattices of groups. 


\section{Contents}

$\begin{array}{ll}\text { Introduction } & 1\end{array}$

1 Some notions and facts 3

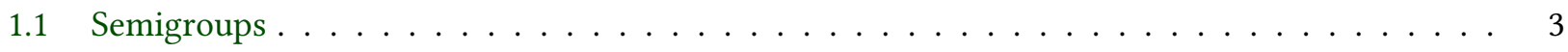

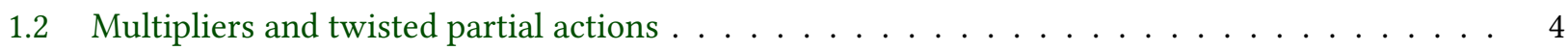

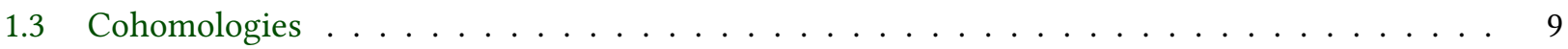

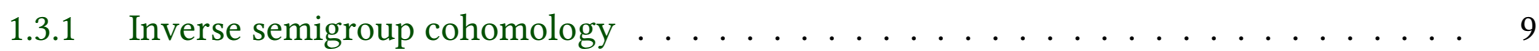

1.3.2 Partial group cohomology . . . . . . . . . . . . . . . . . . . . . 10

1.4 The quotients $\varsigma(A)$, exo $A$, and related partial homomorphisms $\ldots \ldots \ldots 12$

$2 \quad$ Abstract kernel of an extension of $A$ by $G$

$2.1 \quad$ Abstract kernel of an extension of $A$ by $S \ldots \ldots \ldots \ldots \ldots \ldots \ldots$

2.2 From the abstract kernel of an extension of $A$ by $S$ to an abstract kernel of an extension of $A$

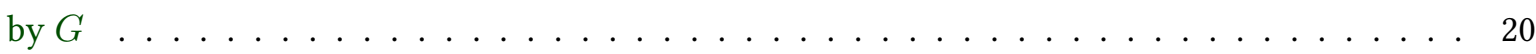

2.3 Obstruction to an extension of an abstract kernel $\ldots \ldots \ldots \ldots \ldots \ldots$

2.4 The description of all the extensions of an abstract kernel $\ldots \ldots \ldots \ldots \ldots$

3 Crossed modules $\quad 33$

3.1 From equivalence classes of crossed module extensions to $H^{3}(T, A) \ldots \ldots \ldots$

3.1.1 Specifications on transversals and cocycles . . . . . . . . . . . . . . . . . 41

3.2 From $H_{\leqslant}^{3}(T, A)$ to equivalence classes of crossed module extensions $\ldots \ldots \ldots$. . . . . . 49

A Crossed modules of groups $\quad 62$

$\begin{array}{ll}\text { Bibliography } & \mathbf{7 0}\end{array}$ 


\section{Introduction}

A partial action of a group on a set can be thought of as a sort of weakening of the usual notion of a group action: instead of associating a (full) bijection to each element of the group, one associates a partial bijection - that is, a bijection between subsets of the set - and, in particular, this means that the composition of two such bijections is a restriction of the bijection associated to the product of the elements of the group, but not necessarily equals to it. One can partially act on sets that have some structure - like rings, semigroups and algebras - and those partial bijections are taken to be isomorphisms between ideals of said structures. Exel studied these partial actions in the context of $C^{*}$-algebras; in particular, he defined the notion of a twisted partial action, giving rise to a restricted form of the 2-cocycle identity, so the development of a corresponding cohomology theory was in order.

Such a cohomology study started in [DK15], in which Dokuchaev and Khrypchenko considered partial actions over commutative monoids, and determined the relations between the already established inverse semigroup cohomology by Lausch in [Lau75]. In particular, they showed that, under some conditions on the module structure of $A$, there is an isomorphism $H^{n}(G, A) \cong H^{n}(\mathcal{S}(G), A)$, for all $n \geqslant 0$, in which the $n$ thcohomology group on the left is their partial cohomology group, and the one on the right is Lausch's inverse semigroup cohomology group. The inverse semigroup $\mathcal{S}(G)$ is the so-called Exel's inverse monoid, defined in [Exe98], and later proved by Kellendonk and Lawson in [KL04] to be isomorphic to the Birget-Rhodes expansion of the group $G$, using Szendrei's results [Sze89].

Since then, this partial cohomology [DK15] was further developed in [DK17, DK18], in particular a more general multiplier-valued partial cohomology was introduced. More recently, Alvares, Alves and Redondo defined another notion of partial group cohomology in [AAR17] based on modules over the partial group algebra. For more information on partial group actions see the survey [Dok19] as well as the book [Exe17].

The partial group cohomology developed in [DK15] and extended in [DK18] matches the usual group cohomology theory in some ways, so it was natural to mirror classical results for this new cohomology. In [DK18], the first and second partial cohomology groups were related to extensions of abelian semilattices of groups by groups. The present thesis original aim was to give the two known interpretations for the third cohomology group in the partial case: the first being the relation with obstructions to extensions of semilattices of groups by groups, the second being the relation with crossed module extensions. The first interpretation is fully developed in this work. With respect to the second one, since partial group cohomology is closely related to inverse semigroup cohomology, it was a necessary step to develop crossed module extensions in the inverse semigroup context - which left the partial case to another time. A detailed exposition of the relation between crossed module extensions of groups and the third usual cohomology group is not easy to find in the literature, so we give it in Appendix A.

After technical notions and some preliminary results in Chapter 1, there are two main parts: Chapter 2 and Chapter 3. The first relates the third partial cohomology group with obstructions to the existence of extensions of semilattices of non-necessarily abelian groups by groups, which realize partial abstract kernels, and, if such extensions exist, classify them by the second partial cohomology group. The latter defines a notion of crossed module over inverse semigroups, relates to it an equivalence class o the 4-term sequences that these crossed modules generate, and gives a bijective correspondence between these equivalence classes and the third order- 
preserving inverse semigroup cohomology group, for the case of a semilattice of groups. 


\section{Some notions and facts}

In this chapter, we present basic notions that will be used in the following chapters, as well as some initial results.

\subsection{Semigroups}

A semigroup is a non-empty set $S$ with an associative binary operation, which will often - if not always - be referred to as the multiplication - of the semigroup. Furthermore, if there is an element $1 \in S$ (an identity element), such that $s \cdot 1=1 \cdot s=s$, for all $s \in S$, then the semigroup is called a monoid.

Given a semigroup $S$, we denote its center by

$$
C(S)=\{s \in S \mid s t=t s, \forall t \in S\}
$$

and, if $S$ is a monoid, we denote its invertible elements by

$$
\mathcal{U}(S)=\left\{s \in S \mid s s^{-1}=s^{-1} s=1\right\}
$$

A semigroup $S$ is called regular, if for any $s \in S$, there exists $s^{-1} \in S$ (an inverse of $s$ ), such that

$$
s s^{-1} s=s \quad \text { and } \quad s^{-1} s s^{-1}=s^{-1} .
$$

A regular semigroup is called inverse if each element has a unique inverse. Inverse semigroups are precisely regular semigroups in which idempotents commute [CP61, Theorem 1.17]. Groups are precisely inverse semigroups that have a unique idempotent.

Every inverse semigroup admits a natural partial order defined by the equivalent equalities

$$
s \leqslant t \Longleftrightarrow \begin{cases}s=e t, & \text { for some idempotent e, or } \\ s=t f, & \text { for some idempotent f, or } \\ s=s s^{-1} t, \text { or } & \\ s=t s^{-1} s . & \end{cases}
$$

The subset of idempotents of a semigroup will be denoted by

$$
\boldsymbol{E}(\boldsymbol{S})=\left\{e \in S \mid e^{2}=e\right\},
$$

and it is a meet semilattice under this partial order: the meet $e \wedge f$ of the elements $e, f \in E(S)$ is their product ef $\in E(S)$.

A semigroup $A$ is called a semilattice of groups if it can be represented as a disjoint union $A=\bigsqcup_{l \in L} A_{l}$, over a semilattice $L$, each $A_{l}$ a subgroup of $A$, and $A_{l} A_{m} \subseteq A_{l \wedge m}$, for all $l, m \in L$. In this case, it follows that $A$ is an inverse semigroup ([CP67, Theorem 7.52]), the semilattice $L$ can be chosen to be $E(A)$, and each group $A_{e}$ is the maximal subgroup of $A$ containing $e$, that is: $A_{e}=\left\{a \in A \mid a a^{-1}=a^{-1} a=e\right\}$. It also 
follows by [CP61, Lemma 4.8] that idempotent elements are central in a semilattice of groups.

As usual, there exists the notion of a homomorphism of semigroups, and taking $\varphi: S \rightarrow S^{\prime}$ a homomorphism of semigroups, if $S$ is an inverse semigroup, then $\varphi(S)$ is also an inverse semigroup, and idempotents of $\varphi(S)$ are images of idempotents of $S$. For the category of inverse semigroups, epimorphisms are exactly surjective homomorphisms and monomorphisms are exactly injective homomorphisms.

A special case of homomorphisms of semigroups are the ones that are injective on idempotents - idempotent-separating homomorphisms. If $\pi: S \rightarrow S^{\prime}$ is an idempotent-separating epimorphism, the inverse image of any $e^{\prime} \in E\left(S^{\prime}\right)$ is a subsemigroup of $S$ that contains exactly one idempotent $e$, with $\pi(e)=e^{\prime}$; thus, it is a group, which we will denote by $K_{e}$. The collection $\left\{K_{e}\right\}_{e \in E(S)}$ of subgroups of $S$ determines a (inverse) subsemigroup $K=\bigsqcup_{e \in E(S)} K_{e}$ of $S$ and hence a semilattice of groups, which is called a (group) kernel normal system. Conversely, for $S$ an arbitrary inverse semigroup, fixing a subgroup $N_{e}$ of $S$ with identity $e$, for each $e \in E(S)$, and considering the collection $\mathcal{K}=\left\{K_{e}\right\}_{e \in E(S)}$, this $\mathcal{K}$ is a kernel normal system of $S$ exactly when $K=\bigsqcup_{e \in E(S)} K_{e}$ is a subsemigroup of $S$ satisfying $s K s^{-1} \subseteq K$ for all $s \in S$ (see [CP67, Theorem 7.54]). (There is a characterization of the kernel normal systems of an arbitrary inverse semigroup in [CP67, p. 60].)

The general case of a kernel normal system of an inverse semigroup is a collection of disjoint subsemigroups (not necessarily subgroups) of $S$ which realize a unique congruence on $S$. A congruence on $S$ is a relation $\rho$ such that

$$
(s, t),\left(s^{\prime}, t^{\prime}\right) \in \rho \Longrightarrow\left(s s^{\prime}, t t^{\prime}\right) \in \rho .
$$

For any homomorphism $\varphi: S \rightarrow S^{\prime}$, the relation $\operatorname{ker} \varphi=\{(s, t) \in S \times S \mid \varphi(s)=\varphi(t)\}$ is called the kernel of $\varphi$, and is in fact a congruence on $S$.

Given a congruence $\rho$ on an inverse semigroup $S$, its kernel is

$$
\operatorname{ker} \rho=E(S / \rho)
$$

and in particular, for a homomorphism $\varphi: S \rightarrow S^{\prime}$ of inverse semigroups the congruence $\operatorname{ker} \varphi$ can be recovered from the set of classes of elements of $S$ which are mapped to idempotents under $\varphi$, thus, $\operatorname{ker} \varphi=$ $\operatorname{ker}(\operatorname{ker} \varphi)$ and $\operatorname{ker} \rho=\operatorname{ker} \rho^{\natural}$, where $\rho^{\natural}$ is the natural epimorphism induced by the congruence $\rho$.

Going back a bit to the case of group kernel normal systems, we can determine precisely what are its classes. The class of the induced congruence $\rho_{\mathcal{K}}$ containing $s \in S$ is $s K_{s^{-1} s}=K_{s s^{-1}} s$ (by [CP67, Theorem 7.55]). In particular, $\rho_{\mathcal{K}}^{\natural}(e)=K_{e}$ for $e \in E(S)$. More precisely, $(s, t) \in \rho_{\mathcal{K}}$ if and only if $t=u s$ for some $u \in K_{s s^{-1}}=$ $K_{t t^{-1}}$, or, equivalently, $t=s v$ for some $v \in K_{s^{-1} s}=K_{t^{-1} t}$. Moreover, such $u$ and $v$ are unique. For if $t=u s$, then $u=u u^{-1} u=u \cdot u u^{-1}=u s s^{-1}=t s^{-1}$. Also, $t=s v$ implies $v=v v^{-1} v=v^{-1} v \cdot v=s^{-1} s v=s^{-1} t$.

\subsection{Multipliers and twisted partial actions}

We shall use several definitions and technical results about multipliers, so all of them are gathered in this subsection. We also introduce the concepts of twisted partial actions, their equivalence, and partial actions.

Definition 1.2.1. A multiplier of a semigroup $S$ is a pair $m$ of maps $s \mapsto m s$ and $s \mapsto s m$ from $S$ to itself, such that for all $s, t \in S$ : 
(i) $m(s t)=(m s) t$;

(ii) $(s t) m=s(t m)$;

(iii) $s(m t)=(s m) t$.

The set of multipliers of $S$, denoted by $\mathcal{M}(S)$, forms a monoid under the usual composition of maps.

Remark 1.2.2. $\quad$ (i) Let $S^{2}=S$. Then for all $m, n \in \mathcal{M}(S)$ and $s \in S$

$$
(m s) n=m(s n) \text {. }
$$

(ii) Let $C(S)^{2}=C(S)$. Then for all $m \in \mathcal{M}(S)$ and $s \in C(S)$

$$
w s=s w
$$

In particular, both items are true, if $S$ is inverse.

Proof: Item (i) is the semigroup version of [DE05, Proposition 2.5 (ii)], while (ii) follows from [DK17, Remark 5.2]. Now, if $S$ is inverse, then $s=s\left(s s^{-1}\right) \in S^{2}$ for any $s \in S$, so $S^{2}=S$. Moreover, $C(S)$ is an inverse subsemigroup of $S$, as for any $s \in C(S)$ and $t \in S$ one has $s^{-1} t=s^{-1}\left(t^{-1}\right)^{-1}=\left(t^{-1} s\right)^{-1}=\left(s t^{-1}\right)^{-1}=$ $t s^{-1}$, whence $s^{-1} \in C(S)$. Thus, $C(S)^{2}=C(S)$ also holds.

Definition 1.2.3. Let $S$ be a semigroup. Define $\phi: S \rightarrow \mathcal{M}(S), s \mapsto \phi_{s}$, to be the multiplier that acts on $t \in S$ as follows:

$$
t \phi_{s}=t s, \phi_{s} t=s t
$$

The multiplier $\phi_{s}$ will be called the inner multiplier ${ }^{1}$ associated with the element $s$ of $S$.

Definition 1.2.4. [DES08, p. 3282] Let $\alpha: S \rightarrow T$ be an isomorphism of semigroups. For any $m \in \mathcal{M}(S)$ denote by $m^{\alpha}$ the multiplier of $T$ acting on $t \in T$ in the following way:

$$
t m^{\alpha}=\alpha\left(\alpha^{-1}(t) m\right), m^{\alpha} t=\alpha\left(m \alpha^{-1}(t)\right)
$$

Remark 1.2.5. It is easily seen that $m \mapsto m^{\alpha}$ is an isomorphism of monoids $\mathcal{M}(S) \rightarrow \mathcal{M}(T)$.

Definition 1.2.6. Let $S^{2}=S$ and $m \in \mathcal{U}(\mathcal{M}(S))$. Denote by $\mu(m)$ the conjugation $s \mapsto m s m^{-1}$ on $S$. By Remark 1.2.2 it is well-defined. Moreover, it is clearly an automorphism of $S$.

Lemma 1.2.7. Let $\alpha: S \rightarrow T$ be an isomorphism of semigroups.

(a) If $\beta: T \rightarrow U$ is another semigroup isomorphism, then

$$
\left(m^{\alpha}\right)^{\beta}=m^{\beta \circ \alpha}
$$

for any $m \in \mathcal{M}(S)$.

(b) If $S^{2}=S$, then

$$
\mu\left(m^{\alpha}\right)=\alpha \circ \mu(m) \circ \alpha^{-1}
$$

\footnotetext{
${ }^{1}$ Petrich [Pet84] calls this an inner bitranslation induced by $s$.
} 
for any $m \in \mathcal{U}(\mathcal{M}(S))$.

Proof: (a) Given $s \in S$, we have

$$
\begin{aligned}
s\left(m^{\alpha}\right)^{\beta} & =\beta\left(\beta^{-1}(s) m^{\alpha}\right)=\beta\left(\alpha\left(\alpha^{-1}\left(\beta^{-1}(s)\right) m\right)\right) \\
& =(\beta \circ \alpha)\left((\beta \circ \alpha)^{-1}(s) m\right)=s m^{\beta \circ \alpha} .
\end{aligned}
$$

Similarly, $\left(m^{\alpha}\right)^{\beta} s=m^{\beta \circ \alpha} s$ :

$$
\begin{aligned}
\left(m^{\alpha}\right)^{\beta} s & =\beta\left(m^{\alpha} \beta^{-1}(s)\right)=\beta\left(\alpha\left(m \alpha^{-1}\left(\beta^{-1}(s)\right)\right)\right) \\
& =(\beta \circ \alpha)\left(m(\beta \circ \alpha)^{-1}(s)\right)=m^{\beta \circ \alpha} s .
\end{aligned}
$$

(b) For arbitrary $t \in T$ we have

$$
\begin{aligned}
\mu\left(m^{\alpha}\right)(t) & =\left(m^{\alpha} t\right)\left(m^{\alpha}\right)^{-1}=\alpha\left(\alpha^{-1}\left(\alpha\left(m \alpha^{-1}(t)\right)\right) m^{-1}\right) \\
& =\alpha\left(m \alpha^{-1}(t) m^{-1}\right)=\left(\alpha \circ \mu(m) \circ \alpha^{-1}\right)(t) .
\end{aligned}
$$

Lemma 1.2.8. Let $S^{2}=S$ and $m \in \mathcal{M}(S)$. Then

$$
m \in C(\mathcal{M}(S)) \Longleftrightarrow \forall s \in S: m s=s m
$$

Proof: The "if" part. Let $m \in C(\mathcal{M}(S))$. Since $S^{2}=S$, any element $s \in S$ is of the form $s=t u$ for some $t, u \in S$. Clearly, $s=\phi_{t} u=t \phi_{u}$, where $\phi_{t}$ and $\phi_{u}$ are the inner multipliers from Definition 1.2.3. Now,

$$
m s=m t u=m \phi_{t} u=\phi_{t} m u=t m \phi_{u}=t \phi_{u} m=t u m=s m .
$$

The "only if" part. Suppose that $m$ "commutes" with any element of $S$. Given $n \in \mathcal{M}(S)$ and $s \in S$, we calculate using Remark 1.2.2 (i)

$$
(m n) s=m(n s)=(n s) m=n(s m)=n(m s)=(n m) s .
$$

Similarly $s(m n)=s(n m)$. Thus, $m n=n m$.

Lemma 1.2.9. Let $S^{2}=S$. Then

$$
m \in C(\mathcal{M}(S)) \Longrightarrow m \in \mathcal{M}(C(S))
$$

Proof: Given $m \in C(\mathcal{M}(S))$ and $s \in C(S)$, we know by Lemma 1.2.8 that $m s=s m$. So, it suffices to show that $m s \in C(S)$. For any $t \in S$ using Lemma 1.2 .8 we have:

$$
(m s) t=m(s t)=m(t s)=(t s) m=t(s m)=t(m s) .
$$


Corollary 1.2.10. Under the conditions of Lemma 1.2.9 if $m \in \mathcal{U}(C(\mathcal{M}(S)))$, then $m \in \mathcal{U}(\mathcal{M}(C(S)))$.

Lemma 1.2.11. Let $S^{2}=S$ and $m, n \in \mathcal{U}(\mathcal{M}(S))$. Then $\mu(m)=\mu(n)$ if and only if there exists $w \in$ $\mathcal{U}(C(\mathcal{M}(S)))$ such that

$$
m=w n
$$

Proof: The "if" part is obvious, so we shall only prove the "only if" part. By definition $\mu(m)=\mu(n)$ means that

$$
m s m^{-1}=n s n^{-1}
$$

for all $s \in S$. So, defining $w:=m n^{-1} \in \mathcal{U}(\mathcal{M}(S))$, we clearly have (1.4) and moreover

$$
w s=s w
$$

for arbitrary $s \in S$. It follows by Lemma 1.2.8 that $w \in C(\mathcal{M}(S))$.

Lemma 1.2.12. Let $S$ be such that $C(S)^{2}=C(S)$. Given $m \in \mathcal{M}(C(S))$ and $n \in \mathcal{M}(S)$, we have

$$
m n=n m \text { on } C(S) .
$$

Proof: Let $s \in C(S)$. Since $m \in \mathcal{M}(C(S))$, it follows that $m s \in C(S)$. Then using Remark 1.2.2 (ii) we have

$$
(n m) s=n(m s)=(m s) n=m(s n)=m(n s)=(m n) s
$$

Equality $s(m n)=s(n m)$ is proved similarly.

Lemma 1.2.13. Let $S^{2}=S$ and $\psi, \varphi: S \rightarrow T$ be two isomorphisms of semigroups. If $\psi=\mu(m) \circ \varphi$ for some $m \in \mathcal{M}(T)$, then

$$
m n^{\varphi}=n^{\psi} m
$$

for all $n \in \mathcal{M}(S)$.

Proof: Given $s \in S$, using Remark 1.2.2 (i) we have

$$
\begin{aligned}
\left(n^{\psi} m\right) s & =n^{\psi}(m s)=n^{\mu(m) \circ \varphi}(m s)=(\mu(m) \circ \varphi)\left(n(\mu(m) \circ \varphi)^{-1}(m s)\right) \\
& =m \varphi\left(n(\mu(m) \circ \varphi)^{-1}(m s)\right) m^{-1}=m \varphi\left(n \varphi^{-1}\left(m^{-1} m s m\right)\right) m^{-1} \\
& =m n^{\varphi}(s m) m^{-1}=m\left(n^{\varphi} s\right) m m^{-1}=\left(m n^{\varphi}\right) s .
\end{aligned}
$$

Similarly, $s\left(n^{\psi} m\right)=s\left(m n^{\varphi}\right)$ :

$$
\begin{aligned}
\left(s n^{\psi}\right) m & =s n^{\mu(m) \circ \varphi} m=\left((\mu(m) \circ \varphi)\left((\mu(m) \circ \varphi)^{-1}(s) n\right) m\right. \\
& =\left(m\left(\varphi\left((\mu(m) \circ \varphi)^{-1}(s) n\right) m^{-1}\right) m=m\left(\left(\varphi\left(\varphi^{-1}\left(m^{-1} s m\right) n\right) m^{-1} m\right)\right.\right. \\
& =m\left(\left(m^{-1} s m\right) n^{\varphi}\right)=\left(m\left(m^{-1} s m\right)\right) n^{\varphi}=(s m) n^{\varphi} .
\end{aligned}
$$


Proposition 1.2.14. Let $A$ be a semilattice of groups. Then there is a one-to-one correspondence between the elements of $\mathcal{M}(C(A))$ and the elements of $C(\mathcal{M}(A))$.

Proof: By Lemma 1.2.9 we know that each $m \in C(\mathcal{M}(A))$ is a multiplier of $C(A)$. It remains to show that each $m \in \mathcal{M}(C(A))$ uniquely extends to $m^{\prime} \in C(\mathcal{M}(A))$. Given such $m$, we shall first prove the following equality

$$
m\left(a a^{-1}\right) a=a\left(a^{-1} a\right) m
$$

and then use it to define an extension of $m$ to a central multiplier on the whole $A$.

Since $A$ is a semilattice of groups, we have $a a^{-1}=a^{-1} a \in C(A)$, so both sides of (1.6) are well-defined, and moreover $m\left(a a^{-1}\right),\left(a^{-1} a\right) m \in C(A)$. Hence, using Remark 1.2 .2 we get

$$
m\left(a a^{-1}\right) a=a m\left(a a^{-1}\right)=a\left(a a^{-1}\right) m=a\left(a^{-1} a\right) m,
$$

proving (1.6). We now define

$$
m^{\prime} a:=m\left(a a^{-1}\right) a
$$

Then

$$
\begin{aligned}
m^{\prime}(a b) & =m\left((a b)(a b)^{-1}\right) a b=m\left(a b b^{-1} a^{-1}\right) a b=m\left(a a^{-1} b b^{-1}\right) a b \\
& =m\left(a a^{-1}\right) b b^{-1} a b=m\left(a a^{-1}\right) a b b^{-1} b=m\left(a a^{-1}\right) a b=\left(m^{\prime} a\right) b,
\end{aligned}
$$

and by symmetry if we put

$$
a m^{\prime}:=a\left(a^{-1} a\right) m
$$

then $(a b) m^{\prime}=a\left(b m^{\prime}\right)$. Furthermore,

$$
\left(a m^{\prime}\right) b=a\left(a^{-1} a\right) m b=a\left(a^{-1} a\right) m b b^{-1} b=a a^{-1} a m\left(b b^{-1}\right) b=a m\left(b b^{-1}\right) b=a\left(m^{\prime} b\right) .
$$

Thus, $m^{\prime} \in \mathcal{M}(A)$. That $m^{\prime} \in C(\mathcal{M}(A))$ follows by (1.6) and Lemma 1.2.8. Clearly, the extension of $m$ is unique, as each $n \in \mathcal{M}(A)$ satisfies $n a=n\left(a a^{-1}\right) a$ and $a n=a\left(a^{-1} a\right) n$.

Definition 1.2.15. A twisted partial action [DES08, DK17] of a group $G$ on a semigroup $A$ is a pair $\Theta=$ $(\theta, w)$, where $\theta=\left\{\theta_{g}: \mathcal{D}_{g^{-1}} \rightarrow \mathcal{D}_{g} \mid g \in G\right\}$ is a collection of isomorphisms between two-sided ideals of $A$ and $w=\left\{w_{g, h} \in \mathcal{M}\left(\mathcal{D}_{g} \mathcal{D}_{g h}\right) \mid g, h \in G\right\}$ satisfying the following properties for all $g, h, k \in G$ :

(TPA1) $\mathcal{D}_{g}^{2}=\mathcal{D}_{g}, \mathcal{D}_{g} \mathcal{D}_{h}=\mathcal{D}_{h} \mathcal{D}_{g}$

(TPA2) $\mathcal{D}_{1}=A, \theta_{1}=\operatorname{id}_{A}$;

(TPA3) $\theta_{g}\left(\mathcal{D}_{g^{-1}} \mathcal{D}_{h}\right)=\mathcal{D}_{g} \mathcal{D}_{g h}$;

(TPA4) $\left(\theta_{g} \circ \theta_{h}\right)(a)=w_{g, h} \theta_{g h}(a) w_{g, h}^{-1}$, for all $a \in \mathcal{D}_{h^{-1}} \mathcal{D}_{h^{-1} g^{-1}}$;

(TPA5) $w_{1, g}=w_{g, 1}=\operatorname{id}_{\mathcal{D}_{g}}$;

(TPA6) $\theta_{g}\left(a w_{h, k}\right) w_{g, h k}=\theta_{g}(a) w_{g, h} w_{g h, k}$, for all $a \in \mathcal{D}_{g^{-1}} \mathcal{D}_{h} \mathcal{D}_{h k}$

Definition 1.2.16. A partial action [DN10, DK15] of a group $G$ on a semigroup $A$ is a collection $\theta=\left\{\theta_{g}\right.$ : $\left.\mathcal{D}_{g^{-1}} \rightarrow \mathcal{D}_{g} \mid g \in G\right\}$ of isomorphisms between two-sided ideals of $A$ satisfying the following properties for 
all $g, h \in G:$

(PA1) $\mathcal{D}_{1}=A, \theta_{1}=\operatorname{id}_{A}$;

(PA2) $\theta_{g}\left(\mathcal{D}_{g^{-1}} \cap \mathcal{D}_{h}\right)=\mathcal{D}_{g} \cap \mathcal{D}_{g h}$

(PA3) $\left(\theta_{g} \circ \theta_{h}\right)(a)=\theta_{g h}(a)$, for all $a \in \mathcal{D}_{h^{-1}} \cap \mathcal{D}_{h^{-1} g^{-1}}$.

Definition 1.2.17. Two twisted partial actions $(\theta, w)$ and $\left(\theta^{\prime}, w^{\prime}\right)$ of $G$ on $A$ are called equivalent, if

(ETPA1) $\mathcal{D}_{g}^{\prime}=\mathcal{D}_{g}$ for all $g \in G$;

and there exists $\varepsilon=\left\{\varepsilon_{g} \in \mathcal{U}\left(\mathcal{M}\left(\mathcal{D}_{g}\right)\right) \mid g \in G\right\}$, such that

(ETPA2) $\theta_{g}^{\prime}(a)=\varepsilon_{g} \theta_{g}(a) \varepsilon_{g}^{-1}$, for all $g \in G$ and $a \in \mathcal{D}_{g^{-1}}$;

(ETPA3) $\theta_{g}^{\prime}(a) w_{g, h}^{\prime} \varepsilon_{g h}=\varepsilon_{g} \theta_{g}\left(a \varepsilon_{h}\right) w_{g, h}$, for all $g, h \in G$ and $a \in \mathcal{D}_{g^{-1}} \mathcal{D}_{h}$.

\subsection{Cohomologies}

In this section, we define the cohomological notions that will be used throughout the thesis.

\subsubsection{Inverse semigroup cohomology}

In this section, we describe inverse semigroup cohomology [Lau75], with results from [DK18], slightly adapting the notations to make them compatible with our own.

Definition 1.3.1. [Lau75, p.274] Let $S$ be an inverse semigroup. A (Lausch's) $\boldsymbol{S}$-module is a semilattice of abelian groups $A$ together with a map $\eta: S \times A \rightarrow A$, denoted by $\eta_{s}(a), s \in S, a \in A$, such that:

(LM1) there is an isomorphism $\theta: E(S) \rightarrow E(A)$;

(LM2) $\eta_{s}(a b)=\eta_{s}(a) \eta_{s}(b)$, for all $s \in S, a, b \in A$;

(LM3) $\eta_{s t}(a)=\eta_{s}\left(\eta_{t}(a)\right)$, for all $s, t \in S, a \in A$;

(LM4) $\eta_{e}(a)=\theta(e) a$, for all $e \in E(S), a \in A$;

(LM5) $\eta_{s}(\theta(e))=\theta\left(\right.$ ses $\left.^{-1}\right)$, for all $s \in S, e \in E(S)$.

Let $A$ be an $S$-module. By adjoining identities $1_{S}$ and $1_{A}$ to $S$ and $A$ we obtain the $S^{1}$-module $A^{1}$ (see [Lau75, p. 285], where one uses the additive notation). In particular, $1_{S}$ acts on $A^{1}$ trivially, and $\eta_{s}\left(1_{A}\right)=\theta\left(s s^{-1}\right)$.

The set of functions

$$
\boldsymbol{C}^{n}\left(\boldsymbol{S}^{\mathbf{1}}, \boldsymbol{A}^{\mathbf{1}}\right):=\left\{f: S^{n} \rightarrow A \mid f\left(s_{1}, \ldots, s_{n}\right) \in A_{\theta\left(s_{1} \ldots s_{n} s_{n}^{-1} \ldots s_{1}^{-1}\right)}\right\}
$$

is an abelian group under the coordinate-wise multiplication, and the following map is a homomorphism, which sends $f \in C^{n}\left(S^{1}, A^{1}\right)$ to $\delta^{n} f \in C^{n+1}\left(S^{1}, A^{1}\right)$, such that

$$
\begin{aligned}
\left(\delta^{n} f\right)\left(s_{1}, \ldots, s_{n+1}\right)= & \eta_{s_{1}}\left(f\left(s_{2}, \ldots, s_{n+1}\right)\right) \\
& \prod_{i=1}^{n} f\left(s_{1}, \ldots, s_{i} s_{i+1}, \ldots, s_{n+1}\right)^{(-1)^{i}} \\
& f\left(s_{1}, \ldots, s_{n}\right)^{(-1)^{n+1}} .
\end{aligned}
$$


The following propositions describe the groups $H^{n}(S, A)$ and $H^{n}\left(S^{1}, A^{1}\right)$.

Proposition 1.3.2. [DK18, Proposition 2.13] Let $S$ be an inverse monoid. For any $S$-module $A$ and for all $n \geqslant 2$ we have

$$
H^{n}(S, A) \cong H^{n}\left(S^{1}, A^{1}\right) .
$$

Proposition 1.3.3. [DK18, Proposition 2.14] Let $S$ be an inverse semigroup and $(\eta, \theta)$ an $S$-module structure on A. Then

$$
\begin{aligned}
& H^{0}\left(S^{1}, A^{1}\right)=0 \\
& H^{1}\left(S^{1}, A^{1}\right) \cong Z^{1}\left(S^{1}, A^{1}\right)=\left\{f: S \rightarrow A \mid \eta_{s}(f(t)) f(s t)^{-1} f(s)=\theta\left(s t t^{-1} s^{-1}\right)\right\} .
\end{aligned}
$$

Moreover, if $S$ is a monoid, then

$$
\begin{aligned}
& H^{0}(S, A) \cong\left\{a \in \mathcal{U}(A) \mid \eta_{s}(a) a^{-1}=\theta\left(s s^{-1}\right), \forall s \in S\right\} \\
& H^{1}(S, A) \cong Z^{1}\left(S^{1}, A^{1}\right) /\left\{f: S \rightarrow A \mid \exists a \in \mathcal{U}(A): f(s)=\eta_{s}(a) a^{-1}, \forall s \in S\right\}
\end{aligned}
$$

The cocycles we work with in Chapter 3 have a property equivalent to being order-preserving. We say that $f \in C^{n}\left(S^{1}, A^{1}\right), n \geqslant 1$, is order-preserving, if

$$
s_{1} \leqslant t_{1}, \ldots, s_{n} \leqslant t_{n} \Longrightarrow f\left(s_{1}, \ldots, s_{n}\right) \leqslant f\left(t_{1}, \ldots, t_{n}\right) .
$$

Since $\leqslant$ respects multiplication, the order-preserving cochains form a subgroup of $C^{n}\left(S^{1}, A^{1}\right)$, denoted by $C_{\leqslant}^{n}\left(S^{1}, A^{1}\right)$. Also, $\delta^{n} f$ preserves the order, whenever $f$ does. So

Proposition 1.3.4. [DK18, Proposition 2.22] The sequence

$$
C_{\leqslant}^{0}\left(S^{1}, A^{1}\right) \stackrel{\delta^{0}}{\rightarrow} \ldots \stackrel{\delta^{n-1}}{\rightarrow} C_{\leqslant}^{n}\left(S^{1}, A^{1}\right) \stackrel{\delta^{n}}{\rightarrow} \ldots
$$

is a cochain complex of abelian groups.

With $C_{\leqslant}^{0}\left(S^{1}, A^{1}\right)$ the abelian group of order-preserving functions $f: E(S) \rightarrow A$, such that $f(e) \in A_{\theta(e)}$ for all $e \in E(S)$. Given $f \in C_{\leqslant}^{0}\left(S^{1}, A^{1}\right)$, the map

$$
\left(\delta^{0} f\right)(s)=\eta_{s}\left(f\left(s^{-1} s\right)\right) f\left(s s^{-1}\right)^{-1}
$$

implies $\delta^{0} f \in C_{\leqslant}^{1}\left(S^{1}, A^{1}\right)$.

Definition 1.3.5. [DK18, Definition 2.23] The groups of $\boldsymbol{n}$-cocycles, $\boldsymbol{n}$-coboundaries and $\boldsymbol{n}$-cohomologies of (1.10) will be denoted by $Z_{\leqslant}^{n}\left(S^{1}, A^{1}\right), B_{\leqslant}^{n}\left(S^{1}, A^{1}\right)$ and $H_{\leqslant}^{n}\left(S^{1}, A^{1}\right)$, respectively $(n \geqslant 0)$.

\subsubsection{Partial group cohomology}

Let $G$ be a group and $A$ a semilattice of groups. If $A$ is commutative, then any twisted partial action (Definition 1.2.15) of $G$ on $A$ splits into a partial action $\theta=\left\{\theta_{x}: \mathcal{D}_{x^{-1}} \rightarrow \mathcal{D}_{x}\right\}_{x \in G}$ (Definition 1.2.16) of $G$ on 
$A$ and a twisting related to $(A, \theta)$, that is, a collection $w=\left\{w_{x, y}\right\}_{x, y \in G}$ of invertible multipliers of $\mathcal{D}_{x} \mathcal{D}_{x y}$, satisfying $w_{1, x}=w_{x, 1}=\operatorname{id}_{\mathcal{D}_{x}}$ and

$$
\theta_{x}\left(\theta_{x^{-1}}(a) w_{y, z}\right) w_{x y, z}^{-1} w_{x, y z} w_{x, y}^{-1}=a, \quad a \in \mathcal{D}_{x} \mathcal{D}_{x y} \mathcal{D}_{x y z}
$$

Now $(\theta, w)$ is equivalent to $\left(\theta^{\prime}, w^{\prime}\right)$ if and only if $\theta=\theta^{\prime}$ and $w$ is equivalent to $w^{\prime}$ in the sense that there exists $\epsilon=\left\{\epsilon_{x} \in \mathcal{U}\left(\mathcal{M}\left(\mathcal{D}_{x}\right)\right)\right\}_{x \in G}$, such that

$$
a w_{x, y}^{\prime}=\theta_{x}\left(\theta_{x^{-1}}(a) \epsilon_{y}\right) \epsilon_{x y}^{-1} \epsilon_{x} w_{x, y}, \quad a \in \mathcal{D}_{x} \mathcal{D}_{x y}
$$

The following are definitions from [DK18]:

Definition 1.3.6. [DK18, Definition 3.2] Let $G$ be a group. A partial $G$-module is a semilattice of abelian groups $A$ with a partial action of $G$ on $A$.

Given a partial $G$-module $(A, \theta)$ and $x_{1}, \ldots, x_{n} \in G$, we write $\mathcal{D}_{x_{1}} \mathcal{D}_{x_{1} x_{2}} \ldots \mathcal{D}_{x_{1} \ldots x_{n}}$ as $\mathcal{D}_{\left(x_{1}, \ldots, x_{n}\right)}$.

Definition 1.3.7. [DK18, Definition 3.3] Let $(A, \theta)$ be a partial $G$-module and $n \geqslant 1$. A partial $\boldsymbol{n}$-cochain of $G$ with values in $A$ is a collection $w=\left\{w\left(x_{1}, \ldots, x_{n}\right)^{-1} i d x_{1}, \ldots, x_{n} \in G\right\}$, where $w\left(x_{1}, \ldots, x_{n}\right) \in$ $\mathcal{U}\left(\mathcal{M}\left(\mathcal{D}_{\left(x_{1}, \ldots, x_{n}\right)}\right)\right)$. By a partial 0-cochain of $G$ with values in $A$ we mean $w \in \mathcal{U}(\mathcal{M}(A))$.

It follows that partial $n$-cochains form an abelian group under pointwise multiplication. This group is denoted by $C^{n}(G, A)$, which is the group of units of

$$
\prod_{\left(x_{1}, \ldots, x_{n}\right) \in G^{n}} \mathcal{M}\left(\mathcal{D}_{\left(x_{1}, \ldots, x_{n}\right)}\right)
$$

Definition 1.3.8. [DK18, Definition 3.5] Given $n \geqslant 1, w \in C^{n}(G, A)$ and $a \in \mathcal{D}_{\left(x_{1}, \ldots, x_{n+1}\right)}$, define

$$
\begin{aligned}
\left(\delta^{n} w\right)\left(x_{1}, \ldots, x_{n+1}\right) a & =\theta_{x_{1}}\left(\theta_{x_{1}^{-1}}(a) w\left(x_{2}, \ldots, x_{n+1}\right)\right) \\
& \prod_{i=1}^{n} w\left(x_{1}, \ldots, x_{i} x_{i+1}, \ldots, x_{n+1}\right)^{(-1)^{i}} \\
& w\left(x_{1}, \ldots, x_{n}\right)^{(-1)^{n+1}} .
\end{aligned}
$$

For $w \in C^{0}(G, A)$ and $a \in \mathcal{D}_{x}$ set

$$
\left(\delta^{0} w\right)(x) a=\theta_{x}\left(\theta_{x^{-1}}(a) w\right) w^{-1} .
$$

Observe that $\theta_{x_{1}^{-1}}(a) \in \mathcal{D}_{x_{1}^{-1}} \mathcal{D}_{\left(x_{2}, \ldots, x_{n+1}\right)}$, so $w\left(x_{2}, \ldots, x_{n+1}\right)$ is applicable in (1.12). The result belongs to $\mathcal{D}_{x_{1}^{-1}} \mathcal{D}_{\left(x_{2}, \ldots, x_{n+1}\right)}$, since the latter is an idempotent ideal. Therefore, $\theta_{x_{1}}\left(\theta_{x_{1}^{-1}}(a) w\left(x_{2}, \ldots, x_{n+1}\right)\right)$ is an element of $\mathcal{D}_{\left(x_{1}, \ldots, x_{n+1}\right)}$. So, the rest of the multipliers in (1.12) are obviously applicable and we have that $\left(\delta^{n} w\right)\left(x_{1}, \ldots, x_{n+1}\right) a$ does in fact belong to $\mathcal{D}_{\left(x_{1}, \ldots, x_{n+1}\right)}$.

And finally:

Lemma 1.3.9. [DK18, Lemma 3.8] For all $n \geqslant 0$ the map $\delta^{n}$ is a homomorphism $C^{n}(G, A) \rightarrow C^{n+1}(G, A)$. 
Corollary 1.3.10. [DK18, Corollary 3.14$]$ The sequence

$$
C^{0}(G, A) \stackrel{\delta^{0}}{\rightarrow} \ldots \stackrel{\delta^{n-1}}{\rightarrow} C^{n}(G, A) \stackrel{\delta^{n}}{\rightarrow} \ldots
$$

is a cochain complex of abelian groups.

Definition 1.3.11. [DK18, Definition 3.15] The complex (1.14) naturally defines the groups of partial $\boldsymbol{n}$ cocycles, $\boldsymbol{n}$-coboundaries and $\boldsymbol{n}$-cohomologies of $G$ with values in $A, n \geqslant 1: Z^{n}(G, A)=\operatorname{ker} \delta^{n}$, $B^{n}(G, A)=\operatorname{im} \delta^{n-1}$ and $H^{n}(G, A)=Z^{n}(G, A) / B^{n}(G, A),\left(H^{0}(G, A)=Z^{0}(G, A)=\operatorname{ker} \delta^{0}\right)$.

\subsection{The quotients $\varsigma(A)$, exo $A$, and related partial homomorphisms}

Throughout this section $A$ will be a semilattice of (not necessarily abelian) groups. Given a set $X$, by $\mathcal{I}(X)$ we denote the symmetric inverse monoid [Law98, p. 6] of $X$.

Remark 1.4.1. If $S$ is an inverse semigroup, then each ideal $I$ of $S$ is idempotent, since each $s \in I$ equals $s \cdot s^{-1} s$ with $s^{-1} s \in I$. It follows that $I \cap J=I J$ for any two non-empty ideals of $S$, since $I \cap J=(I \cap J)^{2} \subseteq$ $I J$, and the converse inclusion holds in an arbitrary semigroup. In particular, any two non-empty ideals of $S$ commute.

Lemma 1.4.2. Let $S$ be a semigroup and $I \triangleleft J \triangleleft S$. If $I^{2}=I$, then $I \triangleleft S$.

Proof: Indeed, $I S=I^{2} S=I \cdot I S \subseteq I \cdot J S \subseteq I J \subseteq I$, and similarly $S I \subseteq I$.

Definition 1.4.3. Given a semigroup $S$, define $\Sigma(S)$ to be the subset of $\mathcal{I}(S)$ that consists of isomorphisms between ideals of $S$.

Proposition 1.4.4. Let $S$ be an inverse semigroup. Then $\Sigma(S)$ is an inverse submonoid of $\mathcal{I}(S)$.

Proof: Given $\varphi, \psi \in \Sigma(S)$, we have:

$$
\varphi \circ \psi: \psi^{-1}(\operatorname{ran} \psi \cap \operatorname{dom} \varphi) \rightarrow \varphi(\operatorname{ran} \psi \cap \operatorname{dom} \varphi)
$$

Observe that $\operatorname{ran} \psi \cap \operatorname{dom} \varphi \triangleleft \operatorname{ran} \psi$, so $\operatorname{dom}(\varphi \circ \psi) \triangleleft \operatorname{dom} \psi$, as $\psi$ is an isomorphism between $\operatorname{dom} \psi$ and $\operatorname{ran} \psi$. Similarly $\operatorname{ran}(\varphi \circ \psi) \triangleleft \operatorname{ran} \varphi$. By Lemma 1.4.2 both $\operatorname{dom}(\varphi \circ \psi)$ and $\operatorname{ran}(\varphi \circ \psi)$ are ideals of $S$, and hence $\varphi \circ \psi \in \Sigma(S)$.

It is obvious that $\Sigma(S)$ is closed with respect to the inverses and contains the identity element of $\mathcal{I}(S)$.

Definition 1.4.5. Let $S$ be an inverse semigroup. Define the following binary relation $\sim$ on $\Sigma(S)$ :

$$
\psi \sim \varphi \Longleftrightarrow\left\{\begin{array}{l}
\operatorname{dom} \varphi=\operatorname{dom} \psi \text { and } \operatorname{ran} \varphi=\operatorname{ran} \psi, \\
\exists m \in \mathcal{U}(\mathcal{M}(\operatorname{ran} \varphi)) \forall s \in \operatorname{dom} \varphi: \psi(s)=m \varphi(s) m^{-1}
\end{array}\right.
$$

By Remarks 1.2.2 and 1.4.1 the expression involving the multiplier $m$ in Definition 1.4.5 makes sense. It is easy to see that $\sim$ is an equivalence: reflexivity and symmetry are obvious, and the transitivity follows from the fact that $\mathcal{M}(\operatorname{ran} \varphi)$ is a monoid. We are going to prove that $\sim$ is in fact a congruence. To this end, we shall need a technical lemma. 
Lemma 1.4.6. Let $S$ be an inverse semigroup. Given $\psi \sim \varphi$ and $I \triangleleft \operatorname{dom} \varphi, J \triangleleft \operatorname{ran} \varphi$, one has

$$
\varphi(I)=\psi(I) \text { and } \varphi^{-1}(J)=\psi^{-1}(J) .
$$

Proof: Let $m \in \mathcal{U}(\mathcal{M}(\operatorname{ran} \varphi))$ be such that $\psi(s)=m \varphi(s) m^{-1}$ for all $s \in \operatorname{dom} \varphi$.

We first prove the assertion for the ideal $I \triangleleft \operatorname{dom} \varphi$. Since $\varphi(I)$ is idempotent thanks to Remark 1.4.1, it follows by [DK17, Remark 5.3] that $m \varphi(I) m^{-1}=\varphi(I)$. But $\psi(I)=m \varphi(I) m^{-1}$, since $\varphi \sim \psi$, whence $\varphi(I)=\psi(I)$.

Now, any $J \triangleleft \operatorname{ran} \varphi$ equals $\varphi\left(\varphi^{-1}(J)\right)$, the latter being $\psi\left(\varphi^{-1}(J)\right)$ by the first equality of the lemma applied to $I=\varphi^{-1}(J) \triangleleft \operatorname{dom} \varphi$. Hence, $\psi^{-1}(J)=\psi^{-1}\left(\psi\left(\varphi^{-1}(J)\right)\right)=\varphi^{-1}(J)$.

Proposition 1.4.7. Let $S$ be an inverse semigroup. Then the equivalence $\sim$ from Definition 1.4.5 is a congruence on $\Sigma(S)$.

Proof: We shall prove that $\sim$ is both left and right compatible. Let $\varphi \sim \psi$ and $\alpha \in \Sigma(A)$. We need to show that $\alpha \circ \varphi \sim \alpha \circ \psi$ and $\varphi \circ \alpha \sim \psi \circ \alpha$.

Recall that

$$
\begin{aligned}
& \alpha \circ \varphi: \varphi^{-1}(\operatorname{ran} \varphi \cap \operatorname{dom} \alpha) \rightarrow \alpha(\operatorname{ran} \varphi \cap \operatorname{dom} \alpha), \\
& \alpha \circ \psi: \psi^{-1}(\operatorname{ran} \psi \cap \operatorname{dom} \alpha) \rightarrow \alpha(\operatorname{ran} \psi \cap \operatorname{dom} \alpha) .
\end{aligned}
$$

The equality $\operatorname{ran}(\alpha \circ \varphi)=\operatorname{ran}(\alpha \circ \psi)$ is clear, $\operatorname{as} \operatorname{ran} \varphi=\operatorname{ran} \psi$. Furthermore, since $\operatorname{ran} \varphi \cap \operatorname{dom} \alpha=$ $\operatorname{ran} \psi \cap \operatorname{dom} \alpha$ is an ideal of $\operatorname{ran} \varphi=\operatorname{ran} \psi$, using Lemma 1.4.6 we have

$$
\begin{aligned}
\operatorname{dom}(\alpha \circ \varphi) & =\varphi^{-1}(\operatorname{ran} \varphi \cap \operatorname{dom} \alpha)=\psi^{-1}(\operatorname{ran} \varphi \cap \operatorname{dom} \alpha) \\
& =\psi^{-1}(\operatorname{ran} \psi \cap \operatorname{dom} \alpha)=\operatorname{dom}(\alpha \circ \psi) .
\end{aligned}
$$

Now let $m \in \mathcal{U}(\mathcal{M}(\operatorname{ran} \varphi))$ such that $\psi(s)=m \varphi(s) m^{-1}$ for all $s \in \operatorname{dom} \varphi$. In view of [DK17, Remark 5.3] the multiplier $m$ of $\operatorname{ran} \varphi$ restricts to a multiplier of $\operatorname{ran} \varphi \cap \operatorname{dom} \alpha \triangleleft \operatorname{ran} \varphi$, which we denote using the same letter. The isomorphism $\alpha$ restricted to $\operatorname{ran} \varphi \cap \operatorname{dom} \alpha$ induces the invertible multiplier $m^{\alpha}$ of $\alpha(\operatorname{ran} \varphi \cap \operatorname{dom} \alpha)$ as in Definition 1.2.4. Then

$$
\begin{aligned}
m^{\alpha}(\alpha \circ \varphi)(x)\left(m^{\alpha}\right)^{-1} & =\alpha\left(m\left(\alpha^{-1} \circ \alpha \circ \varphi\right)(x)\right)\left(m^{\alpha}\right)^{-1}=\alpha(m \varphi(x))\left(m^{\alpha}\right)^{-1} \\
& =\alpha\left(\left(\alpha^{-1} \circ \alpha\right)(m \varphi(x)) m^{-1}\right)=\alpha\left(m \varphi(x) m^{-1}\right)=(\alpha \circ \psi)(x),
\end{aligned}
$$

proving that $\alpha \circ \varphi \sim \alpha \circ \psi$.

The right compatibility of $\sim$ is even easier to prove. We have

$$
\begin{aligned}
& \psi \circ \alpha: \alpha^{-1}(\operatorname{ran} \alpha \cap \operatorname{dom} \psi) \rightarrow \psi(\operatorname{ran} \alpha \cap \operatorname{dom} \psi), \\
& \varphi \circ \alpha: \alpha^{-1}(\operatorname{ran} \alpha \cap \operatorname{dom} \varphi) \rightarrow \varphi(\operatorname{ran} \alpha \cap \operatorname{dom} \varphi)
\end{aligned}
$$

It is evident that $\operatorname{dom}(\psi \circ \alpha)=\operatorname{dom}(\varphi \circ \alpha)$. Since $\operatorname{ran} \alpha \cap \operatorname{dom} \varphi \triangleleft \operatorname{dom} \varphi$, we get $\varphi(\operatorname{ran} \alpha \cap \operatorname{dom} \varphi)=$ $\psi(\operatorname{ran} \alpha \cap \operatorname{dom} \varphi)$ by Lemma 1.4.6, yielding $\operatorname{ran}(\psi \circ \alpha)=\operatorname{ran}(\varphi \circ \alpha)$. Finally, if $s \in \operatorname{dom}(\varphi \circ \alpha)$, then 
$\alpha(s) \in \operatorname{dom} \varphi$, so $\psi(\alpha(s))=m \varphi(\alpha(s)) m^{-1}$, and thus $\varphi \circ \alpha \sim \psi \circ \alpha$.

Definition 1.4.8. Let $S$ be an inverse semigroup. Then the quotient $\Sigma(S) / \sim$ is an inverse monoid, which will be denoted by $\varsigma(S)$.

Remark 1.4.9. The projection homomorphism $\Sigma(S) \rightarrow \varsigma(S)$ is idempotent-separating.

Proof: Indeed, any idempotent of $\Sigma(S)$ is of the form $\mathrm{id}_{I}$, where $I$ is an ideal of $S$. Suppose that $\left[\mathrm{id}_{I}\right]=\left[\operatorname{id}_{J}\right]$ for some ideals $I, J$. Then $\operatorname{id}_{I} \sim \operatorname{id}_{J}$, which implies that $I=\operatorname{dom}\left(\operatorname{id}_{I}\right)=\operatorname{dom}\left(\operatorname{id}_{J}\right)=J$. $\operatorname{Thus} \operatorname{id}_{I}=\operatorname{id}_{J}$.

Recall the following definition from [Lau75].

Definition 1.4.10. Let $A$ be a semilattice of groups. An endomorphism $\varphi: A \rightarrow A$ is called relatively invertible if there exist $\bar{\varphi} \in$ End $A$ and $e_{\varphi} \in E(A)$ satisfying:

(i) $\bar{\varphi} \circ \varphi(a)=e_{\varphi} a$ and $\varphi \circ \bar{\varphi}(a)=\varphi\left(e_{\varphi}\right) a$, for any $a \in A$;

(ii) $e_{\varphi}$ is the identity of $\bar{\varphi}(A)$ and $\varphi\left(e_{\varphi}\right)$ is the identity of $\varphi(A)$.

The set of relatively invertible endomorphisms of $A$ is denoted by end $A$.

Proposition 1.4.11. [DK17, Proposition 3.4] The set end $A$ forms an inverse subsemigroup of $\Sigma(A)$ isomorphic to $\mathcal{I}_{u i}(A)$, the semigroup of isomorphisms between unital ideals of $A$.

Definition 1.4.12. For each fixed $a \in A$, the map $\mu_{a}(b)=a b a^{-1}$ is an endomorphism called inner. It is relatively invertible with $\overline{\mu_{a}}=\mu_{a^{-1}}$ and $e_{\mu_{a}}=a^{-1} a=a a^{-1}$. The set of all relatively invertible inner endomorphisms forms an inverse subsemigroup of end $A$ denoted by in $A$.

Definition 1.4.13. The semigroup in $A$ is a semilattice of groups which determines a kernel normal system of end $A$. The quotient end $A$ /in $A$ will be denoted by exo $A$ and its elements will be called exomorphisms of $A$. Observe that the projection epimorphism end $A \rightarrow$ exo $A$ is idempotent-separating.

The following remark shows that $\sim$ is a generalization of the congruence in $A$ to the case of isomorphisms between non-unital ideals of an inverse semigroup.

Remark 1.4.14. Let $A$ be a semilattice of groups and $\varphi, \psi \in \Sigma(A)$ isomorphisms between unital ideals of $A$. Then, identifying $\varphi$ and $\psi$ with elements of end $A$ as in [DK17, Proposition 3.4], we have $\varphi \sim \psi$ if and only if $(\varphi, \psi) \in$ in $A$. Consequently, $\operatorname{exo}(A)$ can be seen as an inverse subsemigroup of $\varsigma(A)$.

Remark 1.4.15. Let $G$ be a group, $S$ an inverse semigroup and $\Theta=(\theta, w), \Theta^{\prime}=\left(\theta^{\prime}, w^{\prime}\right)$ two twisted partial actions of $G$ on $S$. If $\Theta$ is equivalent to $\Theta^{\prime}$ then $\theta_{g} \sim \theta_{g}^{\prime}$ for all $g \in G$.

Definition 1.4.16. A map $f: G \rightarrow S$ from a group $G$ to a semigroup $S$ is called a partial homomorphism if it satisfies, for all $g, h \in G$ :

$$
\begin{aligned}
& \text { (PH1) } f\left(g^{-1}\right) f(g) f(h)=f\left(g^{-1}\right) f(g h) ; \\
& \text { (PH2) } f(g) f(h) f\left(h^{-1}\right)=f(g h) f\left(h^{-1}\right) ; \\
& \text { (PH3) } f(g) f(1)=f(g) .
\end{aligned}
$$

If $S$ is a monoid, then the partial homomorphism is said to be unital if, instead of (PH3), one has $f(1)=1$. 
Remark 1.4.17. Definition 1.4.16 is a slight modification of what was defined in [DN10], where $S$ was supposed to be a monoid.

The following is well-known.

Corollary 1.4.18. Let $f: G \rightarrow S$ be a partial homomorphism. Then for all $g, h \in G$ :

(i) $f(g) f\left(g^{-1}\right) f(g)=f(g)$;

(ii) $f(1) f(g)=f(g)$;

(iii) $f(g) f(h)=f(g) f\left(g^{-1}\right) f(g h)=f(g h) f\left(h^{-1}\right) f(h)$.

In particular, if $S$ is inverse, then $f\left(g^{-1}\right)=f(g)^{-1}$.

Proof:

(i) Take $h=g^{-1}$ in (PH1): $f(g) f\left(g^{-1}\right) f(g)=f(g) f(1)=f(g)$, and use (PH3) in the last equality.

(ii) Using (PH1) on $f(g) f\left(g^{-1}\right) f(g)$ we have $f(g) f\left(g^{-1}\right) f(g)=f(g) f(1)$, which in turn is equal to $f(g)$, if we use (PH3). Now, if we use (PH2) on $f(g) f\left(g^{-1}\right) f(g)$ we have $f(g) f\left(g^{-1}\right) f(g)=f(1) f(g)$. Putting the two together we get $f(1) f(g)=f(g)$.

(iii) Both equalities are similar, but we prove them both.

We have (PH1):

$$
f\left(x^{-1}\right) f(x y)=f\left(x^{-1}\right) f(x) f(y),
$$

and taking $x=g^{-1}$ and $y=g h$, we have the first equality:

$$
f(g) f\left(g^{-1}\right) f(g h)=f(g) f(h) .
$$

We have (PH2):

$$
f(x y) f\left(y^{-1}\right)=f(x) f(y) f\left(y^{-1}\right),
$$

and taking $x=g h$ and $y=h^{-1}$, we have the second equality:

$$
f(g) f(h)=f(g h) f\left(h^{-1}\right) f(h) .
$$

Within this subsection we shall fix a group $G$, a semilattice of groups $A$ and a partial homomorphism $\psi$ : $G \rightarrow \varsigma(A)$. For any $g \in G$ we denote by $\mathcal{D}_{g}$ the common range of all the isomorphisms of the class $\psi(g)$. We shall also choose a set of representatives $\theta_{g} \in \psi(g)$.

Lemma 1.4.19. The common domain of all the isomorphisms from $\psi(g)$ is $\mathcal{D}_{g^{-1}}$. In particular, $\theta_{g}$ is an isomorphism $\mathcal{D}_{g^{-1}} \rightarrow \mathcal{D}_{g}$.

Proof: Consider the composition $\theta_{g} \theta_{g^{-1}} \in \psi(g) \psi\left(g^{-1}\right)$. Since $\psi(g) \psi\left(g^{-1}\right)=\psi(g) \psi(g)^{-1}$ is an idempotent, it is the class of some idempotent $\operatorname{id}_{I}$ from $\Sigma(A)$. Hence, $\operatorname{dom}\left(\theta_{g} \theta_{g^{-1}}\right)=\operatorname{ran}\left(\theta_{g} \theta_{g^{-1}}\right)=I$. We shall first prove that $I=\mathcal{D}_{g}$. Using the fact that $\psi(g) \psi\left(g^{-1}\right) \psi(g)=\psi(g)$, we have $\operatorname{ran}\left(\theta_{g} \theta_{g^{-1}} \theta_{g}\right)=\operatorname{ran}\left(\theta_{g}\right)=\mathcal{D}_{g}$. It follows that $\mathcal{D}_{g} \subseteq \operatorname{ran}\left(\theta_{g} \theta_{g^{-1}}\right)=I$. On the other hand, $I=\operatorname{ran}\left(\theta_{g} \theta_{g^{-1}}\right) \subseteq \operatorname{ran}\left(\theta_{g}\right)=\mathcal{D}_{g}$, whence $I=\mathcal{D}_{g}$, as desired. 
Clearly, $\operatorname{dom}\left(\theta_{g}\right)=\operatorname{dom}\left(\theta_{g} \theta_{g^{-1}} \theta_{g}\right) \subseteq \operatorname{dom}\left(\theta_{g^{-1}} \theta_{g}\right)=\mathcal{D}_{g^{-1}}$. On the other hand, $\mathcal{D}_{g^{-1}}=\operatorname{dom}\left(\theta_{g^{-1}} \theta_{g}\right) \subseteq$ $\operatorname{dom}\left(\theta_{g}\right)$, proving the lemma.

Lemma 1.4.20. For any $g \in G$ one has

$$
\theta_{g}\left(\mathcal{D}_{g^{-1}} \mathcal{D}_{h}\right)=\mathcal{D}_{g} \mathcal{D}_{g h}
$$

Proof: Indeed, using Lemma 1.4.19 we see that $\theta_{g}\left(\mathcal{D}_{g^{-1}} \mathcal{D}_{h}\right)=\theta_{g}\left(\mathcal{D}_{g^{-1}} \cap \mathcal{D}_{h}\right)=\operatorname{ran}\left(\theta_{g} \theta_{h}\right)$. But $\theta_{g} \theta_{h} \in$ $\psi(g) \psi(h)=\psi(g) \psi\left(g^{-1}\right) \psi(g h)$ and $\operatorname{id}_{\mathcal{D}_{g}} \in \psi(g) \psi\left(g^{-1}\right)$. So, $\operatorname{ran}\left(\theta_{g} \theta_{h}\right)=\operatorname{ran}\left(\operatorname{id}_{\mathcal{D}_{g}} \theta_{g h}\right)=\mathcal{D}_{g} \cap \mathcal{D}_{g h}=$ $\mathcal{D}_{g} \mathcal{D}_{g h}$.

Corollary 1.4.21. We also have a more general equality:

$$
\theta_{g}\left(\mathcal{D}_{g^{-1}} \mathcal{D}_{h_{1}} \ldots \mathcal{D}_{h_{n}}\right)=\mathcal{D}_{g} \mathcal{D}_{g h_{1}} \ldots \mathcal{D}_{g h_{n}}
$$

Proof: For by Remark 1.4 .1 all $\mathcal{D}_{g}$ are idempotent and commute.

Corollary 1.4.22. Let $\psi(G) \subseteq$ exo $A$ and $\mathcal{D}_{g}=1_{g} A$ for some $1_{g} \in E(C(A))$. Then

$$
\theta_{g}\left(1_{g^{-1}} 1_{h}\right)=1_{g} 1_{g h}
$$

Proof: Indeed, $1_{g^{-1}} 1_{h}$ is the identity element of $\mathcal{D}_{g^{-1}} \mathcal{D}_{h}$ and $1_{g} 1_{g h}$ is the identity element of $\mathcal{D}_{g} \mathcal{D}_{h}$.

Lemma 1.4.23. Let $I$ and $J$ be ideals of a semilattice of groups $A$. Then

$$
C(I J)=C(I) C(J)
$$

Proof: The inclusion $C(I) C(J) \subseteq C(I J)$ holds for an arbitrary inverse semigroup $A$. Indeed, given $x \in C(I)$, $y \in C(J)$, and $a \in I \cap J=I J$, we have:

$$
(x y) a=x(y a)=x(a y)=(x a) y=(a x) y=a(x y) .
$$

For the converse inclusion $C(I J) \subseteq C(I) C(J)$, we take $x \in I$ and $y \in J$ such that $x y \in C(I J)$. Then for any $a \in I$ using the fact that $E(A) \subseteq C(A)$, we have:

$$
(x y) a=x y\left(y^{-1} y\right) a=x y\left(a y^{-1} y\right)=\left(a y^{-1} y\right) x y=a x y\left(y^{-1} y\right)=a(x y) .
$$

Hence, $x y \in C(I)$. Similarly, $x y \in C(J)$. Thus, $x y \in C(I) \cap C(J)=C(I) C(J)$, proving $C(I J) \subseteq$ $C(I) C(J)$.

Corollary 1.4.24. The center of an idempotent ideal I of $A$ is also idempotent.

Proof: Indeed, if $I \triangleleft A$ and $I=I^{2}$, then $C(I)=C\left(I^{2}\right)=C(I)^{2}$.

Corollary 1.4.25. For any $g \in G$ we have

$$
\theta_{g}\left(C\left(\mathcal{D}_{g^{-1}}\right) C\left(\mathcal{D}_{h}\right)\right)=C\left(\mathcal{D}_{g}\right) C\left(\mathcal{D}_{g h}\right) .
$$


Proof: Since each $\theta_{g}$ is an isomorphism, it preserves the centers, so using Lemmas 1.4.20 and 1.4.23, we conclude that

$$
\theta_{g}\left(C\left(\mathcal{D}_{g^{-1}}\right) C\left(\mathcal{D}_{h}\right)\right)=\theta_{g}\left(C\left(\mathcal{D}_{g^{-1}} \mathcal{D}_{h}\right)\right)=C\left(\mathcal{D}_{g} \mathcal{D}_{g h}\right)=C\left(\mathcal{D}_{g}\right) C\left(\mathcal{D}_{g h}\right)
$$

Since it is known that a map from a group to an inverse monoid is a partial homomorphism if and only if it is a premorphism, but our definition of a partial homomorphism is a slight modification of the usual one, as stated in Remark 1.4.17, for completion's sake, we shall prove the same equivalence with an inverse semigroup as codomain.

Definition 1.4.26. A map $f: S \rightarrow T$ between inverse semigroups is called a premorphism if it satisfies:

(PM1) $f\left(s^{-1}\right)=f(s)^{-1}, \forall s \in S$;

(PM2) $f(s) f(t) \leqslant f(s t), \forall s, t \in S$.

If $S$ and $T$ are both monoids, then the premorphism is said to be unital if it also satisfies $f(1)=1$.

Remark 1.4.27. A premorphism was called a " $\wedge$-prehomomorphism" in [MR77] or simply a "prehomomorphism" in [Pet84, PR79]. It was also called a "dual prehomomorphism" in [Law98] and a "dual premorphism" in [AS06]. The "duality" taking the form of whether the map satisfies $f(s) f(t) \leqslant f(s t)$ or the converse inequality $f(s t) \leqslant f(s) f(t)$. Also in [MR77], a map satisfying this converse inequality was called a " $v$ prehomomorphism".

The following proof is essentially a repetition of [KL04, Proposition 2.1], with the necessary alterations to the semigroup case.

Theorem 1.4.28. A map $f: G \rightarrow S$ from a group to an inverse semigroup is a premorphism if and only if it is a partial homomorphism.

Proof: Let $f: G \rightarrow S$ be a premorphism. We shall prove (PH1) to (PH3).

For (PH1):

$$
f\left(x^{-1}\right) f(x) f(y) \leqslant f\left(x^{-1}\right) f(x y) \leqslant f(y) .
$$

Now, the inequality $f\left(x^{-1}\right) f(x y) \leqslant f(y)$ implies that $f\left(x^{-1}\right) f(x y)=f(y) e$, for $e \in E(S)$. Multiplying this by $f(y) f\left(y^{-1}\right)$ :

$f(y) f\left(y^{-1}\right) f\left(x^{-1}\right) f(x y)=f(y) f\left(y^{-1}\right) f(y) e=f(y) e$, and we have

$$
f(y) f\left(y^{-1}\right) f\left(x^{-1}\right) f(x y)=f\left(x^{-1}\right) f(x y) .
$$

For the inequality $f\left(x^{-1}\right) f(x) f(y) \leqslant f\left(x^{-1}\right) f(x y)$ :

$$
\begin{aligned}
f\left(x^{-1}\right) f(x) f(y) & =\left(f\left(x^{-1}\right) f(x) f(y)\right)\left(f\left(x^{-1}\right) f(x) f(y)\right)^{-1} f\left(x^{-1}\right) f(x y) \\
& =\left(f\left(x^{-1}\right) f(x)\right)\left(f(y) f(y)^{-1}\right)\left(f(x)^{-1} f(x) f\left(x^{-1}\right)\right) f(x y)
\end{aligned}
$$


but the first two pairs of bracketed elements are idempotents, and idempotents commute in inverse semigroups, and the last three bracketed elements reduce to $f\left(x^{-1}\right)$ using the property of inverses on inverse semigroups and (PM1). Then

$$
\begin{aligned}
f\left(x^{-1}\right) f(x) f(y) & =f(y) f(y)^{-1} f\left(x^{-1}\right) f(x y) \\
& =f\left(x^{-1}\right) f(x y), \text { using (1.15). }
\end{aligned}
$$

The proof for (PH2) is completely analogous, but here it is:

$$
f(x) f(y) f\left(y^{-1}\right) \leqslant f(x y) f\left(y^{-1}\right) \leqslant f(x) .
$$

Now, the inequality $f(x y) f\left(y^{-1}\right) \leqslant f(x)$ implies that $f(x y) f\left(y^{-1}\right)=e f(x)$, for $e \in E(S)$. Multiplying this by $f\left(x^{-1}\right) f(x)$ :

$f(x y) f\left(y^{-1}\right) f\left(x^{-1}\right) f(x)=e f(x) f\left(x^{-1}\right) f(x)=e f(x)$, and we have

$$
f(x y) f\left(y^{-1}\right) f\left(x^{-1}\right) f(x)=f(x y) f\left(y^{-1}\right) .
$$

For the inequality $f(x) f(y) f\left(y^{-1}\right) \leqslant f(x y) f\left(y^{-1}\right)$ :

$$
\begin{aligned}
f(x) f(y) f\left(y^{-1}\right) & =f(x y) f\left(y^{-1}\right)\left(f(x) f(y) f\left(y^{-1}\right)\right)^{-1}\left(f(x) f(y) f\left(y^{-1}\right)\right) \\
& =f(x y)\left(f\left(y^{-1}\right) f(y) f\left(y^{-1}\right)\right)\left(f(x)^{-1} f(x)\right)\left(f(y) f\left(y^{-1}\right)\right)
\end{aligned}
$$

but the last two bracketed pairs of elements are idempotents and idempotents commute in inverse semigroups, and the first three bracketed elements reduce to $f\left(y^{-1}\right)$ using the property of inverses of inverse semigroups and (PM1). Then

$$
\begin{aligned}
f(x) f(y) f\left(y^{-1}\right) & =f(x y) f\left(y^{-1}\right) f(x)^{-1} f(x) \\
& =f(x y) f\left(y^{-1}\right), \text { using }(1.16) .
\end{aligned}
$$

For (PH3), we first observe that (PM1) implies that $f\left(x^{-1}\right)$ is the (semigroup!) inverse of the element $f(x)$. Then

$$
\begin{aligned}
& f(x)=f(x) f\left(x^{-1}\right) f(x) \\
& \leqslant f(x) f(1) \\
& \leqslant f(x) \quad \Longrightarrow f(x) f(1)=f(x) \text {. }
\end{aligned}
$$

Now, let $f: G \rightarrow S$ be a partial homomorphism. We shall prove (PM1) and (PM2).

To prove (PM1), we take $y=x^{-1}$ in (PH2):

$$
f(x) f\left(x^{-1}\right) f(x)=f(x) f(1)=f(x)
$$

and in the last equality was used (PH3). Now let's take $y=x^{-1}$ in (PH1):

$$
f\left(x^{-1}\right) f(x) f\left(x^{-1}\right)=f\left(x^{-1}\right) f(1)=f\left(x^{-1}\right),
$$


and again we used (PH3) in the last equality. Hence $f\left(x^{-1}\right)$ is the inverse of $f(x)$ in the inverse semigroup $S$, thus $f\left(x^{-1}\right)=f(x)^{-1}$.

To prove (PM2), we multiply $f(y)$ on the right of (PH2):

$$
f(x) \underbrace{f(y) f\left(y^{-1}\right) f(y)}_{=f(y)}=f(x y) \underbrace{f\left(y^{-1}\right) f(y)}_{\in E(S)} \leqslant f(x y) .
$$

The following theorems are of note, both for a group $G$ and a set $X$.

Theorem 1.4.29. [Exe98] A map $G \rightarrow \mathcal{I}(X)$ determines a partial action of $G$ on $X$ if and only if it is a partial homomorphism.

Theorem 1.4.30. [KL04] A map $G \rightarrow \mathcal{I}(X)$ determines a partial action of $G$ on $X$ if and only if it is a premorphism. 


\section{Abstract kernel of an extension of $A$ by $G$}

\subsection{Abstract kernel of an extension of $A$ by $S$}

We start by recalling Lausch's construction of the abstract kernel of an extension. Let $A \stackrel{i}{\rightarrow} U \stackrel{j}{\rightarrow} S$ be an extension of a semilattice of (not necessarily abelian) groups $A$ by an inverse semigroup $S$. Then (see [Lau75, p. 291]) there is an idempotent-separating homomorphism $\nu: U \rightarrow$ end $A, u \mapsto \nu_{u}$, defined by

$$
\nu_{u}(a)=i^{-1}\left(u i(a) u^{-1}\right)
$$

Recall from [CP67, Theorem 7.55] that $j(u)=j(v)$ for $u, v \in U$ if and only if there exists $a \in A$ such that $u=i(a) v$ and $i\left(a a^{-1}\right)=v v^{-1}$. Then

$$
\nu_{u}=\nu_{i(a)} \circ \nu_{v}
$$

and $\nu_{i(a)} \in$ in $A$ with $\nu_{i(a)} \circ\left(\nu_{i(a)}\right)^{-1}=\nu_{i\left(a a^{-1}\right)}=\nu_{v v^{-1}}=\nu_{v} \circ\left(\nu_{v}\right)^{-1}$. Therefore, $\nu_{u}$ and $\nu_{v}$ belong to the same class of the idempotent-separating congruence on end $A$ whose kernel coincides with in $A$ ([Lau75, Proposition 8.2 (ii)]). It follows that the map $\psi: S \rightarrow$ exo $A$, which sends $s \in S$ to the in $A$-class $\left[\nu_{u}\right]$ of $\nu_{u}$, where $j(u)=s$, is well-defined, in the sense that the definition does not depend on the choice of $u$. Moreover, for $s=j(u)$ and $t=j(v)$, one has $s t=j(u v)$, and hence

$$
\psi(s t)=\left[\nu_{u v}\right]=\left[\nu_{u} \circ \nu_{v}\right]=\left[\nu_{u}\right]\left[\nu_{v}\right]=\psi(s) \psi(t),
$$

so $\psi$ is a homomorphism. If $\psi(e)=\psi\left(e^{\prime}\right)$ for some $e, e^{\prime} \in E(S)$, then $\left[\nu_{f}\right]=\left[\nu_{f^{\prime}}\right]$ for some (uniquely determined) pair $f, f^{\prime} \in E(U)$ with $j(f)=e$ and $j\left(f^{\prime}\right)=e^{\prime}$. Since $\nu_{f}, \nu_{f^{\prime}} \in E$ (end $A$ ) and the natural epimorphism end $A \rightarrow$ exo $A$ is idempotent-separating by [Lau75, Proposition 8.2 (ii)], we have $\nu_{f}=\nu_{f^{\prime}}$, which implies $f=f^{\prime}$, as $\nu$ is idempotent-separating. Thus, $\psi$ is also idempotent-separating as a homomorphism $S \rightarrow \operatorname{exo} A$.

\subsection{From the abstract kernel of an extension of $A$ by $S$ to an abstract ker- nel of an extension of $A$ by $G$}

Now suppose that the extension $A \stackrel{i}{\rightarrow} U \stackrel{j}{\rightarrow} S$ above admits an order-preserving transversal $\rho: S \rightarrow U$, i.e. $j \circ \rho=\operatorname{id}_{S}$. Then by formulas (7), (9) and (11) from [DK17] the maps $\alpha: E(S) \rightarrow E(A), \lambda: S \rightarrow$ end $A$ and $f: S^{2} \rightarrow A$, where

$$
\begin{aligned}
\alpha(s) & =i^{-1}(\rho(s)), \\
\lambda_{s} & =\nu_{\rho(s)}, \\
f(s, t) & =i^{-1}\left(\rho(s) \rho(t) \rho(s t)^{-1}\right),
\end{aligned}
$$

define a twisted $S$-module structure on $A$. Observe from the definition of $\psi$ that

$$
\psi(s)=\left[\nu_{\rho(s)}\right]=\left[\lambda_{s}\right]
$$


In particular, a choice of another order-preserving transversal $\rho^{\prime}$ leads to the same class $\left[\lambda_{s}\right]$ in exo $A$ for all $s \in S$ (see also [DK17, Proposition 3.10]).

Furthermore, we know by [DK17, Proposition 3.23] that the twisted $S$-module $\Lambda=(\alpha, \lambda, f)$ is Sieben, so by [DK17, Proposition 6.11] it induces a twisted partial action $\Theta=(\theta, w)$ of $\mathcal{G}(S)$ on $A$, where

$$
\begin{aligned}
& \theta_{g}(a)=\lambda_{s}(a), \text { with } s \in g, \alpha\left(s^{-1} s\right)=a a^{-1}, \\
& \left\{\begin{array}{l}
w_{g, h} a=f\left(s, s^{-1} t\right) a \\
a w_{g, h}=a f\left(s, s^{-1} t\right)
\end{array} \text { with } s \in g, t \in g h, \alpha\left(s s^{-1}\right)=\alpha\left(t t^{-1}\right)=a a^{-1} .\right.
\end{aligned}
$$

Moreover, thanks to [DK17, Proposition 6.13] any $\Lambda^{\prime}$ equivalent to $\Lambda$ induces $\Theta^{\prime}$ equivalent to $\Theta$. Thus, in view of Remark 1.4.15, we have a well-defined map that sends $g \in \mathcal{G}(S)$ to $\left[\theta_{g}\right] \in \varsigma(A)$.

Proposition 2.2.1. Given an extension $A \stackrel{i}{\rightarrow} U \stackrel{j}{\rightarrow} S$ and an order-preserving transversal $\rho: S \rightarrow U$ of $j$, the map

$$
\begin{aligned}
\psi: \mathcal{G}(S) & \rightarrow \varsigma(A), \\
g & \mapsto\left[\theta_{g}\right],
\end{aligned}
$$

defined above is a unital partial homomorphism.

Proof: It only suffices to show that for all $g, h \in \mathcal{G}(S)$

$$
\begin{aligned}
\theta_{g^{-1}} \theta_{g} \theta_{h} & \sim \theta_{g^{-1}} \theta_{g h}, \\
\theta_{g} \theta_{h} \theta_{h^{-1}} & \sim \theta_{g h} \theta_{h^{-1}},
\end{aligned}
$$

since $\theta_{1}=\mathrm{id}_{A}$ in view of (TPA2). Observe using (TPA3) that

$$
\begin{aligned}
\operatorname{dom}\left(\theta_{g} \theta_{h}\right) & =\theta_{h}^{-1}\left(\mathcal{D}_{h} \mathcal{D}_{g^{-1}}\right)=\mathcal{D}_{h^{-1}} \mathcal{D}_{h^{-1} g^{-1}}, \\
\operatorname{ran}\left(\theta_{g} \theta_{h}\right) & =\theta_{g}\left(\mathcal{D}_{h} \mathcal{D}_{g^{-1}}\right)=\mathcal{D}_{g} \mathcal{D}_{g h}
\end{aligned}
$$

It follows that

$$
\begin{aligned}
\operatorname{dom}\left(\theta_{g^{-1}} \theta_{g} \theta_{h}\right) & =\operatorname{dom}\left(\theta_{g^{-1}}\left(\theta_{g} \theta_{h}\right)\right)=\theta_{h}^{-1} \theta_{g}^{-1}\left(\mathcal{D}_{g} \mathcal{D}_{g h}\right)=\mathcal{D}_{h^{-1}} \mathcal{D}_{h^{-1} g^{-1}} \\
\operatorname{ran}\left(\theta_{g^{-1}} \theta_{g} \theta_{h}\right) & =\operatorname{ran}\left(\theta_{g^{-1}}\left(\theta_{g} \theta_{h}\right)\right)=\theta_{g^{-1}}\left(\mathcal{D}_{g} \mathcal{D}_{g h}\right)=\mathcal{D}_{g^{-1}} \mathcal{D}_{h}
\end{aligned}
$$

Now,

$$
\begin{aligned}
& \operatorname{dom}\left(\theta_{g^{-1}} \theta_{g h}\right)=\theta_{g h}^{-1}\left(\mathcal{D}_{g h} \mathcal{D}_{g}\right)=\mathcal{D}_{h^{-1} g^{-1}} \mathcal{D}_{h^{-1}} \\
& \operatorname{ran}\left(\theta_{g^{-1}} \theta_{g h}\right)=\theta_{g^{-1}}\left(\mathcal{D}_{g h} \mathcal{D}_{g}\right)=\mathcal{D}_{h} \mathcal{D}_{g^{-1}}
\end{aligned}
$$

so that $\theta_{g^{-1}} \theta_{g} \theta_{h}$ and $\theta_{g^{-1}} \theta_{g h}$ have the same domain and range. For any $a \in \mathcal{D}_{h^{-1} g^{-1}} \mathcal{D}_{h^{-1}}$ by and (TPA4) we have

$$
\theta_{g^{-1}} \theta_{g} \theta_{h}(a)=\left(\theta_{g^{-1}} \theta_{g}\right)\left(\theta_{h}(a)\right)=w_{g^{-1}, g} \theta_{h}(a) w_{g^{-1}, g}^{-1}
$$




$$
\theta_{g^{-1}} \theta_{g h}(a)=w_{g^{-1}, g h} \theta_{h}(a) w_{g^{-1}, g h}^{-1},
$$

whence $\theta_{g^{-1}} \theta_{g} \theta_{h}(a)=w_{g^{-1}, g} w_{g^{-1}, g h}^{-1}\left(\theta_{g^{-1}} \theta_{g h}(a)\right) w_{g^{-1}, g h} w_{g^{-1}, g}^{-1}$, proving (2.2). The proof of (2.3) is similar.

Definition 2.2.2. Given an admissible extension $A \stackrel{i}{\rightarrow} U \stackrel{j}{\rightarrow} G$ of a semilattice of groups $A$ by a group $G$, we define the abstract kernel of this extension as the partial homomorphism

$$
\begin{aligned}
\psi: G & \rightarrow \varsigma(A), \\
g & \mapsto\left[\theta_{g}\right],
\end{aligned}
$$

with $\theta=\left\{\theta_{g}: \mathcal{D}_{g^{-1}} \rightarrow \mathcal{D}_{g}\right\}$ being the action part of the twisted partial action of $G$ on $A$ induced by a refinement $A \stackrel{i}{\rightarrow} U \stackrel{\pi}{\rightarrow} S$ of the extension and an order-preserving transversal $\rho: S \rightarrow U$ of $\pi$.

More generally, by an abstract kernel (without referring to an extension) we mean a triple $(A, G, \psi)$, where $A$ is a semilattice of groups, $G$ is a group, and $\psi: G \rightarrow \varsigma(A)$ is a unital partial homomorphism.

Proposition 2.2.3. Equivalent admissible extensions of $A$ by $G$ define the same abstract kernel $\psi: G \rightarrow \varsigma(A)$.

Proof: By [DK17, Lemma 4.1] equivalent admissible extensions $U$ and $U^{\prime}$ of $A$ by $G$ induce equivalent twisted partial actions $\Theta$ and $\Theta^{\prime}$ of $G$ on $A$. Denoting by $\psi$ and $\psi^{\prime}$ the corresponding abstract kernels, by Remark 1.4.15 we have $\psi(g)=\left[\theta_{g}\right]=\left[\theta_{g}^{\prime}\right]=\psi^{\prime}(g)$.

A general problem of extensions of semilattices of groups by groups is that of constructing all (admissible) extensions with a given abstract kernel $(A, G, \psi)$; that is, constructing all $A \stackrel{i}{\rightarrow} U \stackrel{j}{\rightarrow} G$ whose induced abstract kernel is $\psi$.

\subsection{Obstruction to an extension of an abstract kernel}

Now suppose that we are given an abstract kernel $(A, G, \psi)$. For each $g \in G$, we choose a representative

$$
\theta_{g} \in \psi(g)
$$

and denote by $\mathcal{D}_{g}$ the ideal $\operatorname{ran} \theta_{g}$, so that $\theta_{g}: \mathcal{D}_{g^{-1}} \rightarrow \mathcal{D}_{g}$ by Lemma 1.4.19. It follows from the proof of Lemma 1.4.19 that $\operatorname{id}_{\mathcal{D}_{h^{-1}}} \in \psi\left(h^{-1}\right) \psi(h)$, whence $\theta_{g h} \operatorname{id}_{\mathcal{D}_{h^{-1}}} \in \psi(g h) \psi\left(h^{-1}\right) \psi(h)$. By Corollary 1.4.18 (iii) we have

$$
\theta_{g} \theta_{h} \sim \theta_{g h} \operatorname{id}_{\mathcal{D}_{h^{-1}}}
$$

Hence there exists an invertible multiplier $w_{g, h}$ of $\mathcal{D}_{g} \mathcal{D}_{g h}$ such that

$$
\left(\theta_{g} \theta_{h}\right)(a)=w_{g, h}\left(\theta_{g h} \operatorname{id}_{\mathcal{D}_{h^{-1}}}\right)(a) w_{g, h}^{-1}
$$

for all $a \in \mathcal{D}_{h^{-1}} \mathcal{D}_{h^{-1} g^{-1}}$. Using the notation introduced in Definition 1.2.6 we may write

$$
\left(\theta_{g} \theta_{h}\right)(a)=\mu\left(w_{g, h}\right)\left(\theta_{g h}(a)\right)
$$

for all $a \in \mathcal{D}_{h^{-1}} \mathcal{D}_{h^{-1} g^{-1}}$. 
Lemma 2.3.1. There exists $\beta(g, h, k) \in \mathcal{U}\left(C\left(\mathcal{M}\left(\mathcal{D}_{g} \mathcal{D}_{g h} \mathcal{D}_{g h k}\right)\right)\right)$, such that

$$
w_{h, k}^{\theta_{g}} w_{g, h k}=\beta(g, h, k) w_{g, h} w_{g h, k}
$$

for all $g, h, k \in G$.

Proof: By Lemma 1.4.20 we have dom $\left(\theta_{g} \theta_{h} \theta_{k}\right)=\mathcal{D}_{k^{-1}} \mathcal{D}_{k^{-1} h^{-1}} \mathcal{D}_{k^{-1} h^{-1} g^{-1}}$. Using associativity in $\Sigma(A)$ and (1.3) and (2.5), we compute $\left(\theta_{g} \theta_{h} \theta_{k}\right)(a)$ in two different ways:

$$
\begin{aligned}
\left(\left(\theta_{g} \theta_{h}\right) \theta_{k}\right)(a) & =\mu\left(w_{g, h}\right)\left(\theta_{g h} \theta_{k}(a)\right) \\
& =\left(\mu\left(w_{g, h}\right) \circ \mu\left(w_{g h, k}\right)\right)\left(\theta_{g h k}(a)\right) \\
& =\mu\left(w_{g, h} w_{g h, k}\right)\left(\theta_{g h k}(a)\right), \\
\left(\theta_{g}\left(\theta_{h} \theta_{k}\right)\right)(a) & =\mu\left(w_{h, k}^{\theta_{g}}\right)\left(\theta_{g} \theta_{h k}(a)\right) \\
& =\left(\mu\left(w_{h, k}^{\theta_{g}}\right) \circ \mu\left(w_{g, h k}\right)\right)\left(\theta_{g h k}(a)\right) \\
& =\mu\left(w_{h, k}^{\theta_{g}} w_{g, h k}\right)\left(\theta_{g h k}(a)\right) .
\end{aligned}
$$

Since $\theta_{g h k}(a) \in \mathcal{D}_{g} \mathcal{D}_{g h} \mathcal{D}_{g h k}$ (by Corollary 1.4.21), we have

$$
\mu\left(w_{g, h} w_{g h, k}\right)=\mu\left(w_{h, k}^{\theta_{g}} w_{g, h k}\right) \text { on } \mathcal{D}_{g} \mathcal{D}_{g h} \mathcal{D}_{g h k}
$$

It remains to apply Lemma 1.2.11.

Definition 2.3.2. The map $\beta$ from Lemma 2.3.1 will be called an obstruction to the extension of the abstract kernel $(A, G, \psi)$.

Definition 2.3.3. Given an abstract kernel $(A, G, \psi)$, for each choice of representatives $\theta_{g} \in \psi(g)$ define $\tilde{\theta}_{g}=\left.\theta_{g}\right|_{C\left(\mathcal{D}_{g^{-1}}\right)}: C\left(\mathcal{D}_{g^{-1}}\right) \rightarrow C\left(\mathcal{D}_{g}\right)$.

Remark 2.3.4. In view of Lemma 1.2 .12 the map $\tilde{\theta}_{g}$ does not depend on the choice of representatives $\theta_{g} \in$ $\psi(g)$, and thus is uniquely determined by the abstract kernel $(A, G, \psi)$.

Proposition 2.3.5. The family $\left\{\tilde{\theta}_{g}\right\}_{g \in G}$ from Definition 2.3.3 is a partial action of $G$ on $C(A)$.

Proof: We have $\psi(1)=\left[\operatorname{id}_{A}\right]$, so $\theta_{1}=\mu(m)$ for some $m \in \mathcal{U}(\mathcal{M}(A))$. Then $\tilde{\theta}_{1}=\left.\mu(m)\right|_{C(A)}=\operatorname{id}_{C(A)}$, proving (PA1) for $\tilde{\theta}$.

Property (PA2) for $\tilde{\theta}$ is Corollary 1.4.25.

For (PA3) take $a \in C\left(\mathcal{D}_{h^{-1}} \mathcal{D}_{h^{-1} g^{-1}}\right)$ and write using (2.5) and Corollary 1.4.25 together with Remark 1.2.2 (ii)

$$
\left(\tilde{\theta}_{g} \circ \tilde{\theta}_{h}\right)(a)=\left(\theta_{g} \circ \theta_{h}\right)(a)=w_{g, h} \theta_{g h}(a) w_{g, h}^{-1}=\theta_{g h}(a)=\tilde{\theta}_{g h}(a) .
$$

Lemma 2.3.6. If an abstract kernel $(A, G, \psi)$ has an admissible extension then there exists a choice of representatives $\theta_{g} \in \psi(g)$ such that the corresponding obstruction is the trivial 3-cochain. 
Proof: If $(A, G, \psi)$ is the abstract kernel of an admissible extension of $A$ by $G$, then $\psi(g)=\left[\theta_{g}\right]$, where $(\theta, w)$ is the induced twisted partial action of $G$ on $A$. Observe that (TPA6) is exactly (2.6) with trivial $\beta$. Indeed, $a^{\prime} \in \mathcal{D}_{g} \mathcal{D}_{g h} \mathcal{D}_{g h k}$ if and only if $a^{\prime}=\theta_{g}(a)$ for some $a \in \mathcal{D}_{g^{-1}} \mathcal{D}_{h} \mathcal{D}_{h k}$. Since $a^{\prime} w_{h, k}^{\theta_{g}}=\theta_{g}\left(\theta_{g}^{-1}\left(a^{\prime}\right) w_{h, k}\right)=$ $\theta_{g}\left(a w_{h, k}\right)$, then

$$
a^{\prime} w_{h, k}^{\theta_{g}} w_{g, h k}=a^{\prime} w_{g, h} w_{g h, k}
$$

is equivalent to

$$
\theta_{g}\left(a w_{h, k}\right) w_{g, h k}=\theta_{g}(a) w_{g, h} w_{g h, k}
$$

which is (TPA6).

Lemma 2.3.7. Let $\theta_{g} \in \psi(g), \theta_{g}: \mathcal{D}_{g^{-1}} \rightarrow \mathcal{D}_{g}$, and $w_{g, h} \in \mathcal{U}\left(\mathcal{M}\left(\mathcal{D}_{g} \mathcal{D}_{g h}\right)\right)$ satisfying (2.5). Then

(a) for any $a \in C\left(\mathcal{D}_{g^{-1}} \mathcal{D}_{h} \mathcal{D}_{h k}\right)$ :

$$
\theta_{g}\left(a w_{h, k}\right)=\theta_{g}(a) w_{h, k}^{\theta_{g}}
$$

(b) for any $a \in C\left(\mathcal{D}_{g} \mathcal{D}_{g h} \mathcal{D}_{\text {ghk }} \mathcal{D}_{\text {ghkl }}\right)$ :

$$
a w_{k, l}^{\theta_{g} \theta_{h}}=w_{g, h}\left(a w_{k, l}^{\theta_{g h}}\right) w_{g, h}^{-1}
$$

Proof: (a). To prove (2.7), it suffices to apply (1.1):

$$
\theta_{g}(a) w_{h, k}^{\theta_{g}}=\theta_{g}\left(\theta_{g}^{-1}\left(\theta_{g}(a)\right) w_{h, k}\right)=\theta_{g}\left(a w_{h, k}\right) .
$$

(b). By Proposition 2.3.5 we have

$$
\left(\theta_{g} \theta_{h}\right)^{-1}(a)=\theta_{h}^{-1} \theta_{g}^{-1}(a)=\tilde{\theta}_{h}^{-1} \tilde{\theta}_{g}^{-1}(a)=\tilde{\theta}_{h^{-1} g^{-1}}(a)=\theta_{h^{-1} g^{-1}}(a)
$$

Therefore, using also (1.1) and (2.5) we may write

$$
\begin{aligned}
a w_{k, l}^{\theta_{g} \theta_{h}} & =\left(\theta_{g} \theta_{h}\right)\left(\left(\theta_{g} \theta_{h}\right)^{-1}(a) w_{k, l}\right) \\
& =w_{g, h} \theta_{g h}\left(\theta_{h^{-1} g^{-1}}(a) w_{k, l}\right) w_{g, h}^{-1} \\
& =w_{g, h}\left(a w_{k, l}^{\theta_{g h}}\right) w_{g, h}^{-1}
\end{aligned}
$$

proving (2.8).

The next three lemmas are adaptations of Lemmas IV.8.4-IV.8.6 from [Mac94].

Lemma 2.3.8. Let $(A, G, \psi)$ be an abstract kernel and $\beta$ an obstruction to $(A, G, \psi)$. Then $\beta \in Z^{3}(G, C(A))$, where $C(A)$ is considered as a partial $G$-module via $\tilde{\theta}$. 
Proof: We need to show that $\left(\delta^{3} \beta\right)(g, h, k, l) a=a$ for all $a \in C\left(\mathcal{D}_{g} \mathcal{D}_{g h} \mathcal{D}_{\text {ghk }} \mathcal{D}_{\text {ghkl }}\right)$, that is

$$
\theta_{g}\left(\theta_{g^{-1}}(a) \beta(h, k, l)\right) \beta(g, h k, l) \beta(g, h, k)=a \beta(g h, k, l) \beta(g, h, k l) .
$$

To this end, we express the term $\theta_{g}\left(\theta_{g^{-1}}(a) w_{k, l}^{\theta_{h}} w_{h, k l}\right) w_{g, h k l}$ in two ways using (1.2) and (2.6) to (2.8) and Remark 1.2.2(ii). On the one hand

$$
\begin{aligned}
\theta_{g} & (\theta_{g^{-1}}(a) \underbrace{w_{k, l}^{\theta_{h}} w_{h, k l}}) w_{g, h k l} \\
& =\theta_{g}\left(\theta_{g^{-1}}(a) \beta(h, k, l) w_{h, k} w_{h k, l}\right) w_{g, h k l} \\
& =\theta_{g}\left(\theta_{g^{-1}}(a) \beta(h, k, l)\right) \underbrace{w_{h, k}^{\theta_{g}} w_{g, h k}} w_{g, h k}^{-1} \underbrace{w_{h k, l}^{\theta_{g}} w_{g, h k l}} \\
& =\theta_{g}\left(\theta_{g^{-1}}(a) \beta(h, k, l)\right) \beta(g, h, k) w_{g, h} w_{g h, k} w_{g, h k}^{-1} \beta(g, h k, l) w_{g, h k} w_{g h k, l} \\
& =\theta_{g}\left(\theta_{g^{-1}}(a) \beta(h, k, l)\right) \beta(g, h k, l) \beta(g, h, k) w_{g, h} w_{g h, k} w_{g h k, l} .
\end{aligned}
$$

On the other hand

$$
\begin{aligned}
\theta_{g} & \left(\theta_{g^{-1}}(a) w_{k, l}^{\theta_{h}} w_{h, k l}\right) w_{g, h k l} \\
& =a\left(w_{k, l}^{\theta_{h}}\right)^{\theta_{g}} w_{h, k l}^{\theta_{g}} w_{g, h k l} \\
& =a w_{k, l}^{\theta_{g} \theta_{h}} w_{h, k l}^{\theta_{g}} w_{g, h k l} \\
& =w_{g, h} a \underbrace{w_{k, l}^{\theta_{g h}} w_{g h, k l}} w_{g h, k l}^{-1} w_{g, h}^{-1} \underbrace{w_{h, k l}^{\theta_{g}} w_{g, h k l}} \\
& =w_{g, h} a \beta(g h, k, l) w_{g h, k} w_{g h k, l} w_{g h, k l}^{-1} w_{g, h}^{-1} \beta(g, h, k l) w_{g, h} w_{g h, k l} \\
& =a \beta(g h, k, l) \beta(g, h, k l) w_{g, h} w_{g h, k} w_{g h k, l} .
\end{aligned}
$$

Canceling $w_{g, h} w_{g h, k} w_{g h k, l} \in \mathcal{U}\left(\mathcal{M}\left(\mathcal{D}_{g} \mathcal{D}_{g h} \mathcal{D}_{g h k} \mathcal{D}_{g h k l}\right)\right)$, we get (2.9).

We shall now see how different choices of $\theta$ and $w$ interfere in the obstruction to a given abstract kernel.

Lemma 2.3.9. Another choice of $\left\{w_{g, h}\right\}_{g, h \in G}$ in (2.5), for the same representatives $\left\{\theta_{g}\right\}_{g \in G}$, produces a partial 3 -cocycle cohomologous to $\beta$.

Proof: Let $w_{g, h}^{\prime} \in \mathcal{M}\left(\mathcal{D}_{g} \mathcal{D}_{g h}\right)$ be another multiplier such that

$$
\left(\theta_{g} \theta_{h}\right)(a)=\mu\left(w_{g, h}^{\prime}\right)\left(\theta_{g h}(a)\right)
$$

for all $a \in \mathcal{D}_{h^{-1}} \mathcal{D}_{h^{-1} g^{-1}}$. Since any element of $\mathcal{D}_{g} \mathcal{D}_{g h}$ has the form $\theta_{g h}(a)$ for some $a \in \mathcal{D}_{h^{-1}} \mathcal{D}_{h^{-1} g^{-1}}$, we conclude that $\mu\left(w_{g, h}\right)=\mu\left(w_{g, h}^{\prime}\right)$ on $\mathcal{D}_{g} \mathcal{D}_{g h}$. By Lemma 1.2.11 there exists $v_{g, h} \in \mathcal{U}\left(C\left(\mathcal{M}\left(\mathcal{D}_{g} \mathcal{D}_{g h}\right)\right)\right)$ such that

$$
w_{g, h}^{\prime}=v_{g, h} w_{g, h}
$$

on $\mathcal{D}_{g} \mathcal{D}_{g h}$. Thus, $v$ is a partial 2-cochain, i.e. $v \in C^{2}(G, C(A))$ in the sense of [DK18]. Let $\beta^{\prime}(g, h, k) \in$ $\mathcal{U}\left(C\left(\mathcal{M}\left(\mathcal{D}_{g} \mathcal{D}_{g h} \mathcal{D}_{g h k}\right)\right)\right)$, such that

$$
\left(w_{h, k}^{\prime}\right)^{\theta_{g}} w_{g, h k}^{\prime}=\beta^{\prime}(g, h, k) w_{g, h}^{\prime} w_{g h, k}^{\prime},
$$


which exists in view of Lemma 2.3.1. By (1.2), (2.10) and (2.11) we have

$$
v_{h, k}^{\theta_{g}} w_{h, k}^{\theta_{g}} v_{g, h k} w_{g, h k}=\left(v_{h, k} w_{h, k}\right)^{\theta_{g}} v_{g, h k} w_{g, h k}=\beta^{\prime}(g, h, k) v_{g, h} w_{g, h} v_{g h, k} w_{g h, k} .
$$

Now, since $v_{h, k}$ is a central multiplier of $\mathcal{D}_{h} \mathcal{D}_{h k}$, by Lemma 1.2 .8 its restriction to the ideal $\mathcal{D}_{g^{-1}} \mathcal{D}_{h} \mathcal{D}_{h k}$ is a central multiplier of $\mathcal{D}_{g^{-1}} \mathcal{D}_{h} \mathcal{D}_{h k}$. As $\theta_{g}$ is an isomorphism $\theta_{g}: \mathcal{D}_{g^{-1}} \rightarrow \mathcal{D}_{g}$, it follows that $v_{h, k}^{\theta_{g}}$ is a central multiplier of $\theta_{g}\left(\mathcal{D}_{g^{-1}} \mathcal{D}_{h} \mathcal{D}_{h k}\right)=\mathcal{D}_{g} \mathcal{D}_{g h} \mathcal{D}_{g h k}$. Therefore, in view of Lemma 1.2.12, all the multipliers $v$ commute with all the multipliers $w$ in (2.12) when restricted to $C\left(\mathcal{D}_{g} \mathcal{D}_{g h} \mathcal{D}_{g h k}\right)$. Hence, we may write

$$
v_{h, k}^{\theta_{g}} v_{g, h k} w_{h, k}^{\theta_{g}} w_{g, h k}=\beta^{\prime}(g, h, k) v_{g, h} v_{g h, k} w_{g, h} w_{g h, k} \text { on } C\left(\mathcal{D}_{g} \mathcal{D}_{g h} \mathcal{D}_{g h k}\right)
$$

Applying (2.6) to $w_{h, k}^{\theta_{g}} w_{g, h k}$, we get

$$
v_{h, k}^{\theta_{g}} v_{g, h k} \beta(g, h, k) w_{g, h} w_{g h, k}=\beta^{\prime}(g, h, k) v_{g, h} v_{g h, k} w_{g, h} w_{g h, k} .
$$

Canceling $w_{g, h} w_{g h, k} \in \mathcal{U}\left(\mathcal{D}_{g} \mathcal{D}_{g h} \mathcal{D}_{g h k}\right)$, we come to

$$
v_{h, k}^{\theta_{g}} v_{g, h k} \beta(g, h, k)=\beta^{\prime}(g, h, k) v_{g, h} v_{g h, k} .
$$

Since all the multipliers above are central and invertible on the respective ideal, we finally obtain

$$
\beta^{\prime}(g, h, k)=\beta(g, h, k)\left(\delta^{2} v\right)(g, h, k) \text { on } C\left(\mathcal{D}_{g} \mathcal{D}_{g h} \mathcal{D}_{g h k}\right)
$$

which proves the lemma.

Lemma 2.3.10. Another choice of the representatives $\left\{\theta_{g}\right\}_{g \in G}$ may be followed by a new selection of $\left\{w_{g, h}\right\}_{g, h \in G}$ in (2.5) to induce the same obstruction $\beta$.

Proof: Let $\theta_{g}^{\prime} \in \psi(g)$ be another representative of the class $\psi(g)$. Then $\theta_{g}^{\prime}: \mathcal{D}_{g^{-1}} \rightarrow \mathcal{D}_{g}$ and there exists $m_{g} \in \mathcal{U}\left(\mathcal{M}\left(\mathcal{D}_{g}\right)\right)$

$$
\theta_{g}^{\prime}(a)=\mu\left(m_{g}\right)\left(\theta_{g}(a)\right)
$$

for all $a \in \mathcal{D}_{g^{-1}}$. Taking $a \in \mathcal{D}_{h^{-1}} \mathcal{D}_{h^{-1} g^{-1}}$ we have

$$
\begin{aligned}
\left(\theta_{g}^{\prime} \theta_{h}^{\prime}\right)(a) & =m_{g} \theta_{g}\left(m_{h} \theta_{h}(a) m_{h}^{-1}\right) m_{g}^{-1} \\
& =m_{g} m_{h}^{\theta_{g}} \theta_{g}\left(\theta_{h}(a)\right)\left(m_{h}^{\theta_{g}}\right)^{-1} m_{g}^{-1} \\
& =m_{g} m_{h}^{\theta_{g}} w_{g, h} \theta_{g h}(a) w_{g, h}^{-1}\left(m_{h}^{\theta_{g}}\right)^{-1} m_{g}^{-1} \\
& =m_{g} m_{h}^{\theta_{g}} w_{g, h} m_{g h}^{-1} \theta_{g h}^{\prime}(a) m_{g h} w_{g, h}^{-1}\left(m_{h}^{\theta_{g}}\right)^{-1} m_{g}^{-1} \\
& =\mu\left(w_{g, h}^{\prime}\right)\left(\theta_{g h}^{\prime}(a)\right)
\end{aligned}
$$

where

$$
w_{g, h}^{\prime}:=m_{g} m_{h}^{\theta_{g}} w_{g, h} m_{g h}^{-1} .
$$


Observe using Lemma 1.2.13 that

$$
w_{g, h}^{\prime} m_{g h}=m_{g} m_{h}^{\theta_{g}} w_{g, h}=m_{h}^{\theta_{g}^{\prime}} m_{g} w_{g, h}
$$

Let $\beta^{\prime}$ be the obstruction induced by the pair $\left(\theta^{\prime}, w^{\prime}\right)$. We shall show that $\beta^{\prime}$ in fact coincides with $\beta$, which will prove the lemma. To this end, we compute

$$
\begin{aligned}
& \left(w_{h, k}^{\prime}\right)^{\theta_{g}^{\prime}} \underbrace{w_{g, h k}^{\prime} m_{g h k}}=\left(w_{h, k}^{\prime}\right)^{\theta_{g}^{\prime}} m_{h k}^{\theta_{g}^{\prime}} m_{g} w_{g, h k} \\
& =(\underbrace{w_{h, k}^{\prime} m_{h k}})^{\theta_{g}^{\prime}} m_{g} w_{g, h k} \\
& =\left(m_{k}^{\theta_{h}^{\prime}} m_{h} w_{h, k}\right)^{\theta_{g}^{\prime}} m_{g} w_{g, h k} \\
& =\left(m_{k}^{\theta_{h}^{\prime}}\right)^{\theta_{g}^{\prime}} m_{h}^{\theta_{g}^{\prime}} \underbrace{w_{h, k}^{\theta_{g}^{\prime}} m_{g}} w_{g, h k} \\
& =m_{k}^{\theta_{g}^{\prime} \theta_{h}^{\prime}} m_{h}^{\theta_{g}^{\prime}} m_{g} \underbrace{w_{h, k}^{\theta_{g}} w_{g, h k}} \\
& =m_{k}^{\theta_{g}^{\prime} \theta_{h}^{\prime}} m_{h}^{\theta_{g}^{\prime}} m_{g} \beta(g, h, k) w_{g, h} w_{g h, k} \\
& =\beta(g, h, k) m_{k}^{\theta_{g}^{\prime} \theta_{h}^{\prime}} m_{h}^{\theta_{g}^{\prime}} m_{g} w_{g, h} w_{g h, k} .
\end{aligned}
$$

Then for any $a \in C\left(\mathcal{D}_{g} \mathcal{D}_{g h} \mathcal{D}_{g h k}\right)$ we have

$$
\begin{aligned}
a\left(w_{h, k}^{\prime}\right)^{\theta_{g}^{\prime}} w_{g, h k}^{\prime} m_{g h k} & =\beta(g, h, k) a m_{k}^{\theta_{g}^{\prime} \theta_{h}^{\prime}} m_{h}^{\theta_{g}^{\prime}} m_{g} w_{g, h} w_{g h, k} \\
& =\beta(g, h, k) w_{g, h}^{\prime} a m_{k}^{\theta_{g h}^{\prime}}\left(w_{g, h}^{\prime}\right)^{-1} \underbrace{m_{h}^{\theta_{g}^{\prime}} m_{g} w_{g, h}} w_{g h, k} \\
& =\beta(g, h, k) w_{g, h}^{\prime} a m_{k}^{\theta_{g h}^{\prime}} \underbrace{\left(w_{g, h}^{\prime}\right)^{-1} w_{g, h}^{\prime} m_{g h} w_{g h, k}} \\
& =\beta(g, h, k) w_{g, h}^{\prime} a \underbrace{m_{k}^{\prime}{ }^{m_{g h}} w_{g h, k}} \\
& =\beta(g, h, k) w_{g, h}^{\prime} a w_{g h, k}^{\prime} m_{g h k} \\
& =a \beta(g, h, k) w_{g, h}^{\prime} w_{g h, k}^{\prime} m_{g h k} .
\end{aligned}
$$

Canceling the invertible multiplier $m_{g h k}$, we conclude that

$$
\left(w_{h, k}^{\prime}\right)^{\theta_{g}^{\prime}} w_{g, h k}^{\prime}=\beta(g, h, k) w_{g, h}^{\prime} w_{g h, k}^{\prime}
$$

on $C\left(\mathcal{D}_{g} \mathcal{D}_{g h} \mathcal{D}_{g h k}\right)$. In view of (2.6) the latter means that $\beta^{\prime}(g, h, k)=\beta(g, h, k)$.

Lemma 2.3.11. Let $(A, \theta)$ be a partial $G$-module in the sense of $[D K 18]$ and $v \in C^{2}(G, A)$. Then there exists $v^{\prime} \in C^{2}(G, A)$ such that $\delta^{2} v=\delta^{2} v^{\prime}$ and $v_{1,1}^{\prime}=\operatorname{id}_{A}$.

Proof: Define $u \in C^{1}(G, A)$ by

$$
u_{x} a=a u_{x}=a v_{1,1}^{-1}=v_{1,1}^{-1} a
$$

where $a \in \mathcal{D}_{x}$. Then

$$
a\left(\delta^{1} u\right)_{x, y}=\theta_{x}\left(\theta_{x^{-1}}(a) u_{y}\right) u_{x y}^{-1} u_{x}=\theta_{x}\left(\theta_{x^{-1}}(a) v_{1,1}^{-1}\right) v_{1,1} v_{1,1}^{-1}=\theta_{x}\left(\theta_{x^{-1}}(a) v_{1,1}^{-1}\right)
$$


Now, set $v^{\prime}:=v \cdot \delta^{1} u \in C^{2}(G, A)$. We have $\delta^{2} v^{\prime}=\delta^{2} v \cdot \delta^{2} \delta^{1} u=\delta^{2} v$ and $a v_{1,1}^{\prime}=v_{1,1} \theta_{1}\left(\theta_{1}(a) v_{1,1}^{-1}\right)=a$, as $v_{1,1}$ is a central multiplier of $A$.

We can summarize our results in the following.

Theorem 2.3.12. Given an abstract kernel $(A, G, \psi)$, the center $C(A)$ can be uniquely regarded as a partial $G$ module via $\tilde{\theta}=\left\{\tilde{\theta}_{g}: C\left(\mathcal{D}_{g^{-1}}\right) \rightarrow C\left(\mathcal{D}_{g}\right)\right\}_{g \in G}$, where $\theta_{g} \in \psi(g)$ and $\tilde{\theta}_{g}=\left.\theta_{g}\right|_{C\left(\mathcal{D}_{g^{-1}}\right)}$. Taking the cohomology class of an obstruction $\beta$ to $(A, G, \psi)$, we have a well-defined element $\operatorname{Obs}(A, G, \psi) \in H^{3}(G, C(A))$. The abstract kernel $(A, G, \psi)$ has an admissible extension if and only if $\operatorname{Obs}(A, G, \psi)$ is trivial.

Proof: The "only if" part is explained by Proposition 2.3.5 and Lemmas 2.3.6 and 2.3.8 to 2.3.10.

It remains to prove that the trivial obstruction yields the existence of an extension for $(A, G, \psi)$. Indeed, let $(\theta, w)$ be such that $\beta=\delta^{2} v$. In view of Lemma 2.3.11 we may assume that $v_{1,1}=\mathrm{id}_{A}$. Moreover, since the class of $\beta$ does not depend on the choice of $(\theta, w)$ satisfying (2.4) and (2.5), we may also take $\theta_{1}=\mathrm{id}_{A}$ and $w_{1,1}=\operatorname{id}_{A}$.

By the proof Lemma 2.3.9, if we define $w_{g, h}^{\prime}=v_{g, h}^{-1} w_{g, h}$, then the corresponding $\beta^{\prime}$ satisfies

$$
\beta^{\prime}(g, h, k)=\left(\delta^{2} v\right)(g, h, k)\left(\delta^{2} v^{-1}\right)(g, h, k)=\mathrm{id}_{C\left(\mathcal{D}_{g} \mathcal{D}_{g h} \mathcal{D}_{g h k}\right)} .
$$

It follows from (2.6) that $w^{\prime}$ satisfies (TPA6), and moreover $w_{1,1}^{\prime}=v_{1,1}^{-1} w_{1,1}=\mathrm{id}_{A}$. Writing (TPA6) for the triples $(1,1, g)$ and $(g, 1,1)$ we obtain $w_{1, g}^{\prime}=w_{g, 1}^{\prime}=\operatorname{id}_{\mathcal{D}_{g}}$. Thus, $\left(\theta, w^{\prime}\right)$ is a twisted partial action of $G$ on $A$. It induces the admissible extension $A \stackrel{i}{\rightarrow} A *\left(\theta, w^{\prime}\right) G \stackrel{j}{\rightarrow} G$ constructed in [DK17, Proposition 5.15]. Moreover, by [DK18, Lemma 4.4] there is a refinement $A \stackrel{i}{\rightarrow} A *\left(\theta, w^{\prime}\right) G \stackrel{\pi}{\rightarrow} E(A) *\left(\theta, w^{\prime}\right) G \stackrel{\kappa}{\rightarrow} G$ and an order-preserving transversal $\rho$ of $\pi$, such that the induced twisted partial action of $G$ on $A$ coincides with $\left(\theta, w^{\prime}\right)$. Then the abstract kernel of this extension coincides with $\psi$.

\subsection{The description of all the extensions of an abstract kernel}

Lemma 2.4.1. Let $\theta$ be the action part of a twisted partial action of $G$ on $A, \tilde{\theta}$ the restriction of $\theta$ to $C(A)$, $v \in Z^{2}(G, C(A))$ with respect to $\tilde{\theta}$ and $v^{\prime}=\left\{v_{g, h}^{\prime} \mid g, h \in G\right\}$, where $v_{g, h}^{\prime}$ is the extension of $v_{g, h}$ to a central multiplier of $A$ as in Proposition 1.2.14. Then $\left(\theta, v^{\prime}\right)$ satisfies (TPA6).

Proof: We need to prove that

$$
\left(v_{h, k}^{\prime}\right)^{\theta_{g}} v_{g, h k}^{\prime}=v_{g, h}^{\prime} v_{g h, k}^{\prime}
$$

on $\mathcal{D}_{g} \mathcal{D}_{g h} \mathcal{D}_{g h k}$. An arbitrary element of $\mathcal{D}_{g} \mathcal{D}_{g h} \mathcal{D}_{\text {ghk }}$ has the form $\theta_{g}(a)$, where $a \in \mathcal{D}_{g^{-1}} \mathcal{D}_{h} \mathcal{D}_{h k}$. Applying the left-hand side of (2.16) to $\theta_{g}(a)$ and using (1.1) and (1.8), we have

$$
\begin{aligned}
\theta_{g}(a)\left(v_{h, k}^{\prime}\right)^{\theta_{g}} v_{g, h k}^{\prime} & =\theta_{g}\left(\theta_{g}^{-1}\left(\theta_{g}(a)\right) v_{h, k}^{\prime}\right) v_{g, h k}^{\prime} \\
& =\theta_{g}\left(a v_{h, k}^{\prime}\right) v_{g, h k}^{\prime} \\
& =\theta_{g}\left(a\left(a^{-1} a\right) v_{h, k}\right) v_{g, h k}^{\prime} \\
& =\theta_{g}\left(a\left(a^{-1} a\right) v_{h, k}\right)\left(\theta_{g}\left(a^{-1}\left(a^{-1} a\right) v_{h, k}^{-1}\right) \theta_{g}\left(a\left(a^{-1} a\right) v_{h, k}\right)\right) v_{g, h k}
\end{aligned}
$$

Now, $\theta_{g}\left(a\left(a^{-1} a\right) v_{h, k}\right)=\theta_{g}(a) \tilde{\theta}_{g}\left(\left(a^{-1} a\right) v_{h, k}\right)$, as $a^{-1} a \in C(A)$ and $v_{h, k} \in \mathcal{M}(C(A))$. Moreover, since 
central elements commute with multipliers, we have

$$
\begin{aligned}
\theta_{g}\left(a^{-1}\left(a^{-1} a\right) v_{h, k}^{-1}\right) \theta_{g}\left(a\left(a^{-1} a\right) v_{h, k}\right) & =\tilde{\theta}_{g}\left(a^{-1}\left(a^{-1} a\right) v_{h, k}^{-1} a\left(a^{-1} a\right) v_{h, k}\right) \\
& =\tilde{\theta}_{g}\left(a^{-1} a\left(a^{-1} a\right) v_{h, k}^{-1}\left(a^{-1} a\right) v_{h, k}\right) \\
& =\tilde{\theta}_{g}\left(\left(a^{-1} a\right) v_{h, k}^{-1} v_{h, k}\right) \\
& =\tilde{\theta}_{g}\left(a^{-1} a\right) .
\end{aligned}
$$

Hence,

$$
\begin{aligned}
\theta_{g}(a)\left(v_{h, k}^{\prime}\right)^{\theta_{g}} v_{g, h k}^{\prime} & =\theta_{g}\left(a\left(a^{-1} a\right) v_{h, k}\right) \tilde{\theta}_{g}\left(a^{-1} a\right) v_{g, h k} \\
& =\theta_{g}(a) \tilde{\theta}_{g}\left(\left(a^{-1} a\right) v_{h, k}\right) v_{g, h k} \\
& =\theta_{g}(a) \tilde{\theta}_{g}\left(a^{-1} a\right) v_{h, k}^{\theta_{g}} v_{g, h k} .
\end{aligned}
$$

Similarly,

$$
\theta_{g}(a) v_{g, h}^{\prime} v_{g h, k}^{\prime}=\theta_{g}(a) \tilde{\theta}_{g}\left(a^{-1} a\right) v_{g, h} v_{g h, k} .
$$

It remains to use the partial 2-cocycle identity for $v$.

Definition 2.4.2. Let $\Theta$ be a twisted partial action of $G$ on $A$ and $v \in C^{2}(G, C(A))$. Define

$$
v \Theta=\left(\theta, v^{\prime} w\right)
$$

where $v^{\prime}$ is from 2.4.1.

Following [DK15], a partial 2-cocycle $f$ of $G$ with values in a (not necessarily unital) partial $G$-module $A$ will be called normalized, if $f(1,1)=\operatorname{id}_{A}$. This readily implies that $f(1, g)=f(g, 1)=\operatorname{id}_{\mathcal{D}_{g}}$. As in [DK15, Remark 2.6], one can prove that any partial 2-cocycle is cohomologous to a normalized one. The subgroup of normalized partial 2-cocycles will be denoted by $N Z^{2}(G, A)$.

Lemma 2.4.3. Let $\Theta=(\theta, w)$ be a twisted partial action of $G$ on $A$ and $v \in C^{2}(G, C(A))$. Then $v \Theta$ is also a twisted partial action of $G$ on $A$ if and only if $v$ is a normalized partial 2-cocycle with respect to $\tilde{\theta}$.

Proof: Clearly, $v \Theta$ satisfies (TPA1) to (TPA3) if and only if $\Theta$ does, so we need to prove that $v \Theta$ satisfies (TPA4) to (TPA6) if and only if $v \in N Z^{2}(G, C(A))$.

Let $v \in N Z^{2}(G, C(A))$. Condition (TPA4) for $v \Theta$ follows from Lemma 1.2.8:

$$
\begin{aligned}
\left(v_{g, h}^{\prime} w_{g, h}\right) \theta_{g h}(a)\left(v_{g, h}^{\prime} w_{g, h}\right)^{-1} & =v_{g, h}^{\prime} w_{g, h} \theta_{g h}(a) w_{g, h}^{-1}\left(v_{g, h}^{\prime}\right)^{-1} \\
& =w_{g, h} \theta_{g h}(a) w_{g, h}^{-1} v_{g, h}^{\prime}\left(v_{g, h}^{\prime}\right)^{-1} \\
& =w_{g, h} \theta_{g h}(a) w_{g, h}^{-1} \\
& =\theta_{g} \circ \theta_{h}(a), a \in \mathcal{D}_{h^{-1}} \mathcal{D}_{h^{-1} g^{-1}}
\end{aligned}
$$


For (TPA5) we use the fact that $v$ is normalized:

$$
a\left(v^{\prime} w\right)_{1, g}=a v_{1, g}^{\prime}=a\left(a^{-1} a\right) v_{1, g}=a\left(a^{-1} a\right)=a, a \in \mathcal{D}_{g} .
$$

Finally, for (TPA6) observe that

$$
a\left(v^{\prime} w\right)_{g, h}=\left(a\left(a^{-1} a\right) v_{g, h}\right) w_{g, h}=\left(a^{-1} a\right) v_{g, h} a w_{g, h}, a \in \mathcal{D}_{g} \mathcal{D}_{g h}
$$

so it suffices to apply Lemma 2.4.1.

Conversely, suppose that (TPA4) to (TPA6) hold for $v \Theta$. Condition (TPA5) readily implies that $v_{1,1}=\mathrm{id}_{A}$. It remains to prove that $\left(\delta^{2} v\right)(g, h, k)$ is a trivial multiplier. As it was observed in the proof of Lemma 2.3.6, condition (TPA6) for $\Theta$ is equivalent to

$$
w_{h, k}^{\theta_{g}} w_{g, h k}=w_{g, h} w_{g h, k} \text { on } \mathcal{D}_{g} \mathcal{D}_{g h} \mathcal{D}_{g h k}
$$

Applying this observation to $v \Theta$, we see that

$$
\left(v_{h, k} w_{h, k}\right)^{\theta_{g}} v_{g, h k} w_{g, h k}=v_{g, h} w_{g, h} v_{g h, k} w_{g h, k} \text { on } C\left(\mathcal{D}_{g} \mathcal{D}_{g h} \mathcal{D}_{g h k}\right)
$$

Since the values of $v$ are central multipliers and $\left(v_{h, k} w_{h, k}\right)^{\theta_{g}}=v_{h, k}^{\theta_{g}} w_{h, k}^{\theta_{g}}$ by Remark 1.2.5, we conclude that

$$
v_{h, k}^{\theta_{g}} v_{g, h k}=v_{g, h} v_{g h, k} \text { on } C\left(\mathcal{D}_{g} \mathcal{D}_{g h} \mathcal{D}_{g h k}\right)
$$

the latter being equivalent to $\left(\delta^{2} v\right)(g, h, k)=\operatorname{id}_{C\left(\mathcal{D}_{g} \mathcal{D}_{g h} \mathcal{D}_{g h k}\right)}$.

Lemma 2.4.4. Let $\Theta=(\theta, w)$ be a twisted partial action of $G$ on $A$ and $v \in N Z^{2}(G, C(A))$ with respect to $\tilde{\theta}$. Then $v \Theta$ is equivalent to $\Theta$ if and only if $v \in B^{2}(G, C(A))$.

Proof: Using Definition 1.2.17, we see that $v \Theta$ is equivalent to $\Theta$ if and only if there exists $\varepsilon_{g} \in \mathcal{U}\left(\mathcal{M}\left(\mathcal{D}_{g}\right)\right)$, such that

$$
\begin{aligned}
\theta_{g}(a) & =\varepsilon_{g} \theta_{g}(a) \varepsilon_{g}^{-1} \text { for all } a \in \mathcal{D}_{g^{-1}} \\
\theta_{g}(a)\left(\theta_{g}\left(a^{-1} a\right) v_{g, h}\right) w_{g, h} \varepsilon_{g h} & =\varepsilon_{g} \theta_{g}\left(a \varepsilon_{h}\right) w_{g, h} \text { for all } a \in \mathcal{D}_{g^{-1}} \mathcal{D}_{h} .
\end{aligned}
$$

By Lemma 1.2.8 condition (2.18) is equivalent to the fact that $\varepsilon_{g} \in \mathcal{U}\left(C\left(\mathcal{M}\left(\mathcal{D}_{g}\right)\right)\right)$. It follows that $w_{g, h} \varepsilon_{g h}=$ $\varepsilon_{g h} w_{g, h}$ on $\mathcal{D}_{g} \mathcal{D}_{g h}$, so that we may cancel $w_{g, h}$ in (2.19) to get

$$
\theta_{g}(a)\left(\theta_{g}\left(a^{-1} a\right) v_{g, h}\right) \varepsilon_{g h}=\varepsilon_{g} \theta_{g}\left(a \varepsilon_{h}\right) \text { for all } a \in \mathcal{D}_{g^{-1}} \mathcal{D}_{h}
$$

This is equivalent to

$$
a\left(a^{-1} a\right) v_{g, h}=\theta_{g}\left(\theta_{g}^{-1}(a) \varepsilon_{h}\right) \varepsilon_{g h}^{-1} \varepsilon_{g} \text { for all } a \in \mathcal{D}_{g} \mathcal{D}_{g h}
$$

If $a \in C\left(\mathcal{D}_{g} \mathcal{D}_{g h}\right)$, then we obtain

$$
a v_{g, h}=a\left(\delta^{1} \varepsilon\right)(g, h)
$$


i.e. $v \in B^{2}(G, C(A))$.

Conversely, if $v=\delta^{1} \varepsilon$ and $a \in \mathcal{D}_{g}$, then writing (2.21) for $a^{-1} a \in C\left(\mathcal{D}_{g}\right)$ and multiplying by $a$ on the left we obtain (2.20), which gives (2.19).

Lemma 2.4.5. Let $\Theta=(\theta, w)$ and $\Theta^{\prime}=\left(\theta^{\prime}, w^{\prime}\right)$ be two twisted partial actions of $G$ on $A$ with the same domains $\left\{\mathcal{D}_{g}\right\}_{g \in G}$, such that (2.13) and (2.14) hold for some $m_{g} \in \mathcal{U}\left(\mathcal{M}\left(\mathcal{D}_{g}\right)\right)$. Then $\Theta$ and $\Theta^{\prime}$ are equivalent.

Proof: It suffices to show that (2.14) implies

$$
\theta_{g}^{\prime}(a) w_{g, h}^{\prime} m_{g h}=m_{g} \theta_{g}\left(a m_{h}\right) w_{g, h} \text { for all } a \in \mathcal{D}_{g^{-1}} \mathcal{D}_{h}
$$

Multiplying both sides of (2.14) by $m_{g h}$ on the right and applying them to $\theta_{g}^{\prime}(a), a \in \mathcal{D}_{g^{-1}} \mathcal{D}_{h}$, on the left, we obtain

$$
\begin{aligned}
\theta_{g}^{\prime}(a) w_{g, h}^{\prime} m_{g h} & =\theta_{g}^{\prime}(a) m_{h}^{\theta_{g}^{\prime}} m_{g} w_{g, h} \\
& =\theta_{g}^{\prime}\left(a m_{h}\right) m_{g} w_{g, h} \\
& =m_{g} \theta_{g}\left(a m_{h}\right) w_{g, h}
\end{aligned}
$$

Theorem 2.4.6. If an abstract kernel $(A, G, \psi)$ has an admissible extension, then the set of equivalence classes of admissible extensions of $(A, G, \psi)$ is in a one-to-one correspondence with the set $H^{2}(G, C(A))$.

Proof: We shall adapt the proof of [Mac94, Theorem IV.8.8], namely, we shall prove that $H^{2}(G, C(A))$ acts transitively and freely on the set of equivalence classes of admissible extensions of $A$ by $G$.

By [DK17, Theorem 6.12] it is enough to consider the classes of admissible extensions of the form

$$
A \rightarrow A *_{\Theta} G \rightarrow G
$$

where $\Theta=(\theta, w)$ is a twisted partial action of $G$ on $A$.

To define the action of $H^{2}(G, C(A))$, we represent an element of $H^{2}(G, C(A))$ as the class $[v]$ of $v \in$ $N Z^{2}(G, C(A))$. Then we define the result of the action of $[v]$ on the class of the extension (2.22) as being the class of

$$
A \rightarrow A *_{v \Theta} G \rightarrow G
$$

where $v \Theta$ is given by (2.17). In view of Lemma 2.4.3 this is well defined.

Let us prove that the action is transitive. Given two twisted partial actions $\Theta=(\theta, w)$ and $\Theta^{\prime}=\left(\theta^{\prime}, w^{\prime}\right)$ of $G$ on $A$, such that $\psi$ is the abstract kernel of both $A *_{\Theta} G$ and $A *_{\Theta^{\prime}} G$ seen as extensions of $A$ by $G$, we have $\left[\theta_{g}\right]=\psi(g)=\left[\theta_{g}^{\prime}\right]$ in $\varsigma(A)$. Then there is $m_{g} \in \mathcal{U}\left(\mathcal{M}\left(\mathcal{D}_{g}\right)\right)$, such that (2.13) holds. Let $w_{g, h}^{\prime \prime}$ be defined by the right hand side of (2.14). By Lemma 2.3.10 the pair $\left(\theta^{\prime}, w^{\prime \prime}\right)$ determines the same (trivial) obstruction as $\Theta$, i.e. it is a twisted partial action of $G$ on $A$. Moreover, $\left(\theta^{\prime}, w^{\prime \prime}\right)$ is equivalent to $\Theta$ by Lemma 2.4.5. On the other hand, by the proof of Lemma 2.3.10 we see that

$$
\mu\left(w_{g, h}^{\prime}\right)\left(\theta_{g h}^{\prime}(a)\right)=\left(\theta_{g}^{\prime} \theta_{h}^{\prime}\right)(a)=\mu\left(w_{g, h}^{\prime \prime}\right)\left(\theta_{g h}^{\prime}(a)\right) \text { for all } a \in \mathcal{D}_{g^{-1}} \mathcal{D}_{h^{-1} g^{-1}}
$$


Hence, by Lemma 1.2.11 there is $v_{g, h} \in \mathcal{U}\left(C\left(\mathcal{M}\left(\mathcal{D}_{g} \mathcal{D}_{g h}\right)\right)\right)$, such that $w_{g, h}^{\prime \prime}=v_{g, h} w_{g, h}^{\prime}$. It follows that $\Theta$ is equivalent to $v \Theta^{\prime}$, where $v \in N Z^{2}(G, C(A))$ thanks to Lemma 2.4.3. Thus, $A *_{\Theta} G$ and $A *_{v \Theta^{\prime}} G$ are equivalent as extensions of $A$ by $G$ in view of [DK18, Lemma 4.7].

We now prove that the action is free. Let $\Theta=(\theta, w)$ be a twisted partial action of $G$ on $A$, such that $A *_{\Theta} G$ and $A *_{v \Theta} G$ are equivalent as extensions of $A$ by $G$. Then $\Theta$ is equivalent to $v \Theta$ by [DK18, Lemmas 4.1 and 4.4]. Thus, $v \in B^{2}(G, C(A))$ thanks to Lemma 2.4.4. 


\section{Crossed modules}

\subsection{From equivalence classes of crossed module extensions to $H^{3}(T, A)$}

In this section, we define the notion of a crossed module over an inverse semigroup, relate to it a 4-term sequence, associate an equivalence class of such sequences and an element of $H^{3}(T, A)$. To start, recall some definitions from [DK17, Lau75].

Definition 3.1.1. [DK17, Definition 3.8] Let $S$ be an inverse semigroup. A twisted $\boldsymbol{S}$-module is a semilattice of groups $A$ with a triple $\Lambda=(\alpha, \lambda, f)$, where $\alpha: E(S) \rightarrow E(A)$ is an isomorphism, $\lambda: S \rightarrow$ end $A$ is a map, and $f: S \times S \rightarrow A$ is a map such that $f(s, t) \in A_{\alpha\left(s t t^{-1} s^{-1}\right)}$ (called a twisting) satisfying:

(TM1) $\lambda_{e}(a)=\alpha(e) a$, for all $e \in E(S)$ and $a \in A$;

(TM2) $\lambda_{s}(\alpha(e))=\alpha\left(\right.$ ses $\left.^{-1}\right)$, for all $s \in S, e \in E(S)$;

(TM3) $\lambda_{s} \circ \lambda_{t}(a)=f(s, t) \lambda_{s t}(a) f(s, t)^{-1}$, for all $s, t \in S$ and $a \in A$;

(TM4) $f(s e, e)=\alpha\left(s e s^{-1}\right), f(e, e s)=\alpha\left(e s s^{-1}\right)$, for all $s \in S, e \in E(S)$;

(TM5) $\lambda_{s}(f(t, u)) f(s, t u)=f(s, t) f(s t, u)$, for all $s, t, u \in S$.

Remark 3.1.2. For the case to be defined of crossed modules the twisting will be taken trivial: $f(s, t)=$ $\alpha\left(s t t^{-1} s^{-1}\right)$, for all $s, t \in S$. As a consequence of (TM3), the map $\lambda$ is in fact a homomorphism.

Definition 3.1.3. [Lau75, p.274] Let $S$ be an inverse semigroup. A Lausch's $\boldsymbol{S}$-module is a semilattice of abelian groups $A$ together with a map $\eta: S \times A \rightarrow A$, denoted by $\eta_{s}(a), s \in S, a \in A$, such that:

(LM1) there is an isomorphism $\theta: E(S) \rightarrow E(A)$;

(LM2) $\eta_{s}(a b)=\eta_{s}(a) \eta_{s}(b)$, for all $s \in S, a, b \in A$;

(LM3) $\eta_{s t}(a)=\eta_{s}\left(\eta_{t}(a)\right)$, for all $s, t \in S, a \in A$;

(LM4) $\eta_{e}(a)=\theta(e) a$, for all $e \in E(S), a \in A$;

(LM5) $\eta_{s}(\theta(e))=\theta\left(\right.$ ses $\left.^{-1}\right)$, for all $s \in S, e \in E(S)$.

Lemma 3.1.4. For each $s \in S$, the map $\eta_{s}: A \rightarrow A$ of a Lausch's module structure is relatively invertible.

Proof: We will show that each $\eta_{s}$ is an isomorphism for the ideals

$$
\eta_{s}: \theta\left(s^{-1} s\right) A \rightarrow \theta\left(s s^{-1}\right) A,
$$

which implies that $e_{\eta_{s}}=\theta\left(s^{-1} s\right)$, and its relative inverse is $\bar{\eta}_{s}=\eta_{s^{-1}}$.

First, for $s \in S$ and $a \in A_{\theta(e)}$ :

$$
\begin{aligned}
\eta_{s}(a) & =\eta_{s}\left(a a^{-1} a\right)=\eta_{s}\left(a a^{-1}\right) \eta_{s}(a) \\
& =\eta_{s}(\theta(e)) \eta_{s}(a)=\theta\left(\operatorname{ses}^{-1}\right) \eta_{s}(a) .
\end{aligned}
$$

This means that composing $\eta_{s^{-1}}$ and $\eta_{s}$ we get:

$$
\begin{aligned}
\eta_{s^{-1}} \circ \eta_{s}(a) & =\eta_{s^{-1}}\left(\theta\left(\operatorname{ses}^{-1}\right) \eta_{s}(a)\right)=\eta_{s^{-1}}\left(\theta\left(\operatorname{ses}^{-1}\right)\right) \eta_{s^{-1} s}(a) \\
& =\theta\left(s^{-1} \operatorname{ses}^{-1} s\right) \theta\left(s^{-1} s\right) a
\end{aligned}
$$

(by (LM4) and (LM5)) 


$$
=\theta\left(s^{-1} s e\right) a=\theta\left(s^{-1} s\right) \theta(e) a=\theta\left(s^{-1} s\right) a a^{-1} a=\theta\left(s^{-1} s\right) a,
$$

and

$$
\begin{aligned}
\eta_{s} \circ \eta_{s^{-1}}(a) & =\eta_{s}\left(\theta\left(s^{-1} e s\right) \eta_{s^{-1}}(a)\right)=\eta_{s}\left(\theta\left(s^{-1} e s\right)\right) \eta_{s s^{-1}}(a) \\
& =\theta\left(s s^{-1} e s s^{-1}\right) \theta\left(s s^{-1}\right) a \\
& =\theta\left(s s^{-1} e\right) a=\theta\left(s s^{-1}\right) \theta(e) a=\theta\left(s s^{-1}\right) a a^{-1} a=\theta\left(s s^{-1}\right) a .
\end{aligned}
$$

And since $\eta_{s}\left(\theta\left(s^{-1} s\right)\right)=\theta\left(s s^{-1} s s^{-1}\right)=\theta\left(s s^{-1}\right)$, by (LM5), we see that $\theta\left(s^{-1} s\right)$ is the identity element of $\bar{\eta}_{s}(A)=\eta_{s^{-1}}(A)$.

Proposition 3.1.5. A Lausch's module structure is equivalent to a twisted module structure with trivial twisting on abelian semilattices of groups.

Proof: Fixing the inverse semigroup $S$ and the semilattice of abelian groups $A$, if $A$ is a Lausch's $S$-module, the map $\eta$ can be seen as $\eta: S \rightarrow$ end $A$ (by Lemma 3.1.4), and it is a homomorphism by (LM3), as well as each $\eta_{s}$ by (LM2). With no further inquiries, (TM1) is equivalent to (LM4), (TM2) is equivalent to (LM5), and the existence of $\alpha$ for a twisted $S$-module structure is equivalent to (LM1).

Starting with the twisted module structure, by [DK17, Remark 3.9], each $\lambda_{s}$ is relatively invertible for the ideals $\lambda_{s}: \alpha\left(s^{-1} s\right) A \rightarrow \alpha\left(s s^{-1}\right) A$, satisfying (LM2). A trivial twisting in a twisted $S$-module structure means $f(s, t)=\alpha\left(s t t^{-1} s^{-1}\right)$, for all $s, t \in S$. So, (TM3) becomes $\lambda_{s} \circ \lambda_{t}(a)=\alpha\left(s t t^{-1} s^{-1}\right) \lambda_{s t}(a) \alpha\left(s t t^{-1} s^{-1}\right)^{-1}=$ $\lambda_{s t}(a)$, since $\alpha\left(s t t^{-1} s^{-1}\right)$ is the identity on $\lambda_{s t}(A)$. That is, $\lambda: S \rightarrow$ end $A$ is a homomorphism, and (LM3) is satisfied.

With a trivial twisting, both (TM4) and (TM5) become blank statements, since

$$
\lambda_{s}\left(\alpha\left(t u u^{-1} t^{-1}\right)\right) \alpha\left(\text { stuu }^{-1} t^{-1} s^{-1}\right)=\alpha\left(\text { stuu }^{-1} t^{-1} s^{-1}\right) \alpha\left(\text { stuu }^{-1} t^{-1} s^{-1}\right)=\alpha\left(\text { stuu }^{-1} t^{-1} s^{-1}\right),
$$

and

$$
\alpha\left(s t t^{-1} s^{-1}\right) \alpha\left(s t u u^{-1} t^{-1} s^{-1}\right)=\alpha\left(s t u u^{-1} t^{-1} s^{-1}\right) .
$$

This last proposition is here to justify the interchangeable use of both the expressions for module structures on abelian semilattices of groups.

Definition 3.1.6. Let $S$ be an inverse semigroup. A crossed $\boldsymbol{S}$-module is a semilattice of groups $N$ with a triple $(\lambda, \alpha, \beta)$, where $\lambda: S \rightarrow$ end $N$ is a homomorphism, $\alpha: E(S) \rightarrow E(N)$ is an isomorphism, and $\beta: N \rightarrow S$ is a homomorphism such that $\left.\beta\right|_{E(N)}=\alpha^{-1}$, satisfying:

(CM1) $\lambda_{e}(n)=\alpha(e) n$, for all $e \in E(S), n \in N$;

(CM2) $\lambda_{s}(\alpha(e))=\alpha\left(s^{-1}\right)$, for all $s \in S, e \in E(S)$;

(CM3) $\lambda_{\beta(n)}\left(n^{\prime}\right)=n n^{\prime} n^{-1}$, for all $n, n^{\prime} \in N$;

(CM4) $\beta\left(\lambda_{s}(n)\right)=s \beta(n) s^{-1}$, for all $s \in S, n \in N$. 
Remark 3.1.7. The pair $(\lambda, \alpha)$ satisfying (CM1) and (CM2) means that $N$ is a twisted $S$-module with trivial twisting. The property (CM4) means that the homomorphism $\beta$ is equivariant with respect to the conjugation action of $S$ on itself, and (CM3) is called Peiffer identity in the literature, for the case of groups (see [Bro82]).

Example 3.1.8. (a) Taking $N$ a semilattice of groups and $S=$ end $N$, if $S \ni \varphi: e_{\varphi} N \rightarrow \varphi\left(e_{\varphi}\right) N$, we define $\lambda_{\varphi}(n):=\varphi\left(e_{\varphi} n\right)$ and take $\beta_{n} \in S$ the conjugation by $n: \beta_{n}\left(n^{\prime}\right)=n n^{\prime} n^{-1}$. Then (CM3) is true by definition, and for (CM4), taking $\varphi \in$ end $N$ :

$$
\begin{aligned}
& \beta\left(\lambda_{\varphi}(n)\right)\left(n^{\prime}\right)=\beta\left(\varphi\left(e_{\varphi} n\right)\right)\left(n^{\prime}\right) \quad\left(\varphi \beta_{n} \bar{\varphi}\right)\left(n^{\prime}\right)=\varphi\left(\beta_{n}\left(\bar{\varphi}\left(n^{\prime}\right)\right)\right) \\
& =\varphi\left(e_{\varphi} n\right) n^{\prime} \varphi\left(e_{\varphi} n\right)^{-1} ; \quad=\varphi\left(n \bar{\varphi}\left(n^{\prime}\right) n^{-1}\right) \\
& =\varphi(n) \varphi \circ \bar{\varphi}\left(n^{\prime}\right) \varphi\left(n^{-1}\right) \\
& =\varphi(n) \varphi\left(e_{\varphi}\right) n^{\prime} \varphi(n)^{-1} \\
& =\varphi\left(e_{\varphi} n\right) n^{\prime} \varphi\left(e_{\varphi} n\right)^{-1} \text {. }
\end{aligned}
$$

(b) Any abelian $S$-module $N$, in the sense of Definition 3.1.3, has a homomorphism $\lambda: S \rightarrow$ end $N$ and an isomorphism $\alpha=\theta: E(S) \rightarrow E(N)$. It remains to define $\beta: N \rightarrow S$ by $\beta(n)=\alpha^{-1}\left(n n^{-1}\right)$.

Equality $(\mathrm{CM} 3)$ is: $\lambda_{\beta(n)}\left(n^{\prime}\right)=\lambda_{\alpha^{-1}\left(n n^{-1}\right)}\left(n^{\prime}\right) \stackrel{(\mathrm{LM} 4)}{=} \alpha\left(\alpha^{-1}\left(n n^{-1}\right)\right) n^{\prime}=n n^{-1} n^{\prime}=n n^{\prime} n^{-1}$, and this last equality holds since $N$ is abelian.

$$
\begin{aligned}
\text { Equality }(\mathrm{CM} 4) \text { is: } \beta\left(\lambda_{s}(n)\right) & =\alpha^{-1}\left(\lambda_{s}(n) \lambda_{s}(n)^{-1}\right) \\
& =\alpha^{-1}\left(\lambda_{s}\left(n n^{-1}\right)\right) \\
& =\alpha^{-1}\left(\lambda_{s}(\alpha(e))\right) \\
& =\alpha^{-1}\left(\alpha\left(s e s^{-1}\right)\right) \\
& =s e s^{-1} \\
& =s \alpha^{-1}\left(n n^{-1}\right) s^{-1} \\
& =s \beta(n) s^{-1}
\end{aligned}
$$

(c) Any group kernel normal system $N$ for an inverse semigroup $S$ is a crossed module with $S$ acting by conjugation and $\beta$ being the inclusion.

Lemma 3.1.9. Given $N$ a crossed $S$-module, then $A:=\beta^{-1}(E(S))$ is a semilattice of groups, central in $N$; this semilattice can also be seen as the kernel of $\beta$.

Proof: Since $\beta$ is an isomorphism on idempotents, we have $A$ a semilattice of groups $\bigsqcup_{e \in E(N)} A_{e}$, with $A_{e}:=$ $\{a \in N \mid \beta(a)=\beta(e)\}$.

For each $e \in E(N)$, we have $\lambda_{\beta(e)}(n)=e n e^{-1}=e n$, by $(\mathrm{CM} 3)$.

Now, given $a \in A_{e}$, we have $\lambda_{\beta(a)}(n)=a n a^{-1}$. Since $\beta(a)=\beta(e)$, we conclude $e n=a n a^{-1}, \forall n \in N$.

Thus, $e n a=a n a^{-1} a \Longrightarrow n a=a n, \forall n \in N$; which implies $A \subseteq C(N)$.

Since $s \beta(N) s^{-1} \subseteq \beta(N)$, by (CM4), and $\beta(N)$ is a subsemigroup of $S$, we have $\mathcal{B}=\left\{\beta\left(N_{e}\right)\right\}_{e \in E(N)}$ a group kernel normal system for $S$, by [CP67, Theorem 7.54]. Setting $T:=S / \rho_{\mathcal{B}}$, we have $\pi=\rho_{\mathcal{B}}^{\natural}: S \rightarrow T$ an idempotent-separating epimorphism, and $\pi^{-1}(E(T))=\beta(N)$.

Lemma 3.1.10. The crossed $S$-module structure $(\lambda, \alpha, \beta)$ on $N$ induces a Lausch's $T$-module structure on $A$.

Proof: Considering the functions $i: A \rightarrow N$, the inclusion, $\pi: S \rightarrow T$, the projection, define the function $\eta: T \times A \rightarrow A$ by $\eta(x, a) \mapsto \eta_{x}(a):=\lambda_{s}(i(a))$, such that $\pi(s)=x, s \in S$.

This definition does not depend on the choice of $s \in S$ such that $\pi(s)=x$. Since, if $\pi(s)=\pi(t)$, then $(s, t) \in \operatorname{ker} \pi \Longleftrightarrow t=s \beta(n)$, for $n \in N_{\beta^{-1}\left(s^{-1} s\right)}=N_{\alpha\left(s^{-1} s\right)}$, meaning $n n^{-1}=\alpha\left(s^{-1} s\right)$. 
And $\lambda_{t}(i(a))=\lambda_{s}(i(a))$ :

$$
\begin{aligned}
\lambda_{t}(i(a)) & =\lambda_{s \beta(n)}(i(a))=\lambda_{s} \lambda_{\beta(n)}(i(a)) \\
& \stackrel{(C M 3)}{=} \lambda_{s}\left(n i(a) n^{-1}\right)=\lambda_{s}\left(n n^{-1} i(a)\right)=\lambda_{s}\left(n n^{-1}\right) \lambda_{s}(i(a))=\lambda_{s}\left(\alpha\left(s^{-1} s\right)\right) \lambda_{s}(i(a)) \\
& =\alpha\left(s s^{-1} s s^{-1}\right) \lambda_{s}(i(a))=\alpha\left(s s^{-1}\right) \lambda_{s}(i(a))=\lambda_{s}(i(a)) .
\end{aligned}
$$

Now, for the Lausch's module properties:

(LM1) The map $\theta: E(T) \rightarrow E(A)$ is an isomorphism, since $i, \alpha, \pi$ are isomorphisms.

$$
e \mapsto i^{-1}\left(\alpha\left(\pi^{-1}(e)\right)\right)
$$

(LM2) For $a \in A$ and $x \in T$ and $s \in S$ such that $\pi(s)=x: \quad \eta_{x}(a b)=\lambda_{s}(i(a b))$

$$
\begin{aligned}
& =\lambda_{s}(i(a) i(b)) \\
& =\lambda_{s}(i(a)) \lambda_{s}(i(b)) \\
& =\eta_{x}(a) \eta_{x}(b) .
\end{aligned}
$$

(LM3) For $a \in A, x, y \in T$ and $s, t \in S$ such that $\pi(s)=x$ and $\pi(t)=y$ we have $\pi(s t)=x y$ and:

$$
\begin{aligned}
\eta_{x y}(a) & =\lambda_{s t}(i(a)) \\
& =\lambda_{s}\left(\lambda_{t}(i(a))\right) \\
& =\eta_{x}\left(\eta_{y}(a)\right) .
\end{aligned}
$$

(LM4) For $a \in A, e \in E(T)$ and $e^{\prime} \in E(S)$ such that $\pi\left(e^{\prime}\right)=e: \eta_{e}(a)=\lambda_{e^{\prime}}(i(a))$

$$
\begin{aligned}
& =\alpha\left(e^{\prime}\right) i(a) \\
& =\alpha\left(\pi^{-1}(e)\right) i(a) \\
& =i\left(i^{-1}\left(\alpha\left(\pi^{-1}(e)\right)\right) a\right) \\
& =i(\theta(e) a) .
\end{aligned}
$$

(LM5) For $e \in E(A), x \in T$ and $s \in S$ such that $\pi(s)=x$ we have: $\eta_{x}(\theta(e))=\lambda_{s}(i(\theta(e)))$

$$
\begin{aligned}
& =\lambda_{s}\left(\alpha\left(p i^{-1}(e)\right)\right) \\
& =\alpha\left(s \pi^{-1}(e) s^{-1}\right) \\
& =\alpha\left(\pi^{-1}\left(x e x^{-1}\right)\right) \\
& =i\left(\theta\left(x e x^{-1}\right)\right) .
\end{aligned}
$$

Thus we have a 4-term sequence

$$
A \stackrel{i}{\rightarrow} N \stackrel{\beta}{\rightarrow} S \stackrel{\pi}{\rightarrow} T
$$

with $i$ a monomorphism, $\pi$ an idempotent-separating epimorphism, $i(A)=\beta^{-1}(E(S)), \beta(N)=\pi^{-1}(E(T))$, $A$ a Lausch's $T$-module (as in Definition 3.1.3), and $N$ a crossed $S$-module (as in Definition 3.1.6). We have, in particular, that the three homomorphisms are isomorphisms on idempotents.

The goal of this chapter is to show that sequences of this form are classified (up to an equivalence relation) by $H^{3}(T, A)$, provided $T$ is a semilattice of groups.

Taking an arbitrary inverse semigroup $T$ and an arbitrary Lausch's $T$-module $A$, consider all possible sequences like (3.1), with $N$ a crossed $S$-module such that its structure $(\lambda, \alpha, \beta)$ induces the given Lausch's module structure $(\eta, \theta)$ of $T$ on $A$. Impose on these sequences the smallest equivalent relation such that

$$
A \stackrel{i}{\rightarrow} N \stackrel{\beta}{\rightarrow} S \stackrel{\pi}{\rightarrow} T
$$


is equivalent to

$$
A \stackrel{i^{\prime}}{\rightarrow} N^{\prime} \stackrel{\beta^{\prime}}{\rightarrow} S^{\prime} \stackrel{\pi^{\prime}}{\rightarrow} T
$$

whenever there is a commutative diagram with homomorphisms $\varphi_{1}$ and $\varphi_{2}$ (not necessarily isomorphisms!):

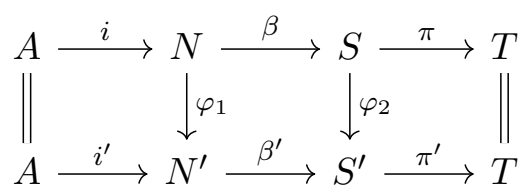

in which the vertical arrows are compatible with the module structures $S$ and $S^{\prime}$ on $N$ and $N^{\prime}$, that is, for $s \in S, n \in N$ :

$$
\varphi_{1}\left(\lambda_{s}(n)\right)=\lambda_{\varphi_{2}(s)}\left(\varphi_{1}(n)\right)
$$

We denote the set of these equivalence classes as $\mathcal{E}(T, A)$.

The next results are to prove the existence of a well-defined function from $\mathcal{E}(T, A)$ to $H^{3}(T, A)$.

Proposition 3.1.11. Any exact sequence (3.1) determines an element in $H^{3}(T, A)$.

Proof: Given an exact sequence like (3.1), choose a function $\rho: T \rightarrow S$ such that $\pi \circ \rho=\mathrm{id}_{T}$. Since $\pi(\rho(x) \rho(y))=\pi(\rho(x y))$, there exists a function $f: T \times T \rightarrow \beta(N)=\operatorname{ker} \pi$ such that

$$
\rho(x) \rho(y)=f(x, y) \rho(x y)
$$

with $f(x, y) \in \beta(N)_{\rho(x y) \rho(x y)^{-1}}$.

Using the associativity of the product in $\beta(N) \subseteq S$ and the fact that $\beta(N)$ is a semilattice of groups:

$$
\begin{array}{rll}
-\rho(x)(\rho(y) \rho(z)) & =\rho(x) f(y, z) \rho(y z) & \\
& =\rho(x) f(y, z) \rho(x)^{-1} \rho(x) \rho(y z) & \text { since } \rho(x)^{-1} \rho(x) \text { is an idempotent } \\
& =\rho(x) f(y, z) \rho(x)^{-1} f(x, y z) \rho(x y z) ; \\
& & \\
& &
\end{array}
$$

we get

$$
\rho(x) f(y, z) \rho(x)^{-1} f(x, y z)=f(x, y) f(x y, z) .
$$

Since $f(x, y) \in \beta(N)_{\rho(x y) \rho(x y)^{-1}}$, we can lift $f$ to a function $F: T \times T \rightarrow N$, by

$$
\beta(F(x, y))=f(x, y)
$$

with $F(x, y) \in N_{\beta^{-1}\left(\rho(x y) \rho(x y)^{-1}\right)}$.

Right-hand side of (3.5) is $f(x, y) f(x y, z)=\beta(F(x, y)) \beta(F(x y, z))$

$$
=\beta(F(x, y) F(x y, z)) \text {; }
$$

Left-hand side of (3.5) is $\rho(x) f(y, z) \rho(x)^{-1} f(x, y z)=\rho(x) \beta(F(y, z)) \rho(x)^{-1} \beta(F(x, y z))$

$$
\begin{array}{ll}
\stackrel{(\mathrm{CM} 4)}{=} & \beta\left(\lambda_{\rho(x)}(F(y, z))\right) \beta(F(x, y z)) \\
= & \beta\left(\lambda_{\rho(x)}(F(y, z)) F(x, y z)\right) .
\end{array}
$$


This means that $\left(\lambda_{\rho(x)}(F(y, z)) F(x, y z), F(x, y) F(x y, z)\right) \in \operatorname{ker} \beta=i(A)$ and there exists a function $c:$ $T \times T \times T \rightarrow A$ such that

$$
\lambda_{\rho(x)}(F(y, z)) F(x, y z)=i(c(x, y, z)) F(x, y) F(x y, z)
$$

with $c(x, y, z) \in A_{(\beta \circ i)^{-1}\left(\rho(x y z) \rho(x y z)^{-1}\right)}=A_{\theta\left(\rho(x y z) \rho(x y z)^{-1}\right)}$.

We now show that $c$ is a 3-cocycle, whose cohomology class is independent of the choices of $F$ and $\rho$, and this cohomology class is the desired element of $H^{3}(T, A)$.

- To show that $c$ is a 3-cocycle, we express

$$
\lambda_{\rho(x)}\left[\lambda_{\rho(y)}(F(z, w)) F(y, z w)\right] F(x, y z w)
$$

in two ways; first computing the bracketed elements:

$$
\begin{aligned}
& \lambda_{\rho(x)}\left[\lambda_{\rho(y)}(F(z, w)) F(y, z w)\right] F(x, y z w) \\
& \stackrel{(3.7)}{=} \lambda_{\rho(x)}[i(c(y, z, w)) F(y, z) F(y z, w)] F(x, y z w) \\
& \quad=\lambda_{\rho(x)}(i(c(y, z, w)) \underbrace{\lambda_{\rho(x)}(F(y, z))} \underbrace{\lambda_{\rho(x)}(F(y z, w)) F(x, y z w)} \\
& \quad \stackrel{(3.7)}{=} \lambda_{\rho(x)}\left(i(c(y, z, w)) i(c(x, y, z)) F(x, y) F(x y, z) F(x, y z)^{-1} i(c(x, y z, w)) F(x, y z) F(x y z, w)\right. \\
& \quad=\lambda_{\rho(x)}\left(i(c(y, z, w)) i(c(x, y, z)) i(c(x, y z, w)) F(x, y) F(x y, z) F(x, y z)^{-1} F(x, y z) F(x y z, w)\right. \\
& \quad=\lambda_{\rho(x)}(i(c(y, z, w)) i(c(x, y, z)) i(c(x, y z, w)) F(x, y) F(x y, z) F(x y z, w) ;
\end{aligned}
$$

now using the fact that $\lambda_{\rho(x)}$ is a homomorphism:

$$
\begin{aligned}
& \lambda_{\rho(x)}\left[\lambda_{\rho(y)}(F(z, w)) F(y, z w)\right] F(x, y z w) \\
& \quad=\lambda_{\rho(x)}(\lambda_{\rho(y)}(F(z, w)) \underbrace{\lambda_{\rho(x)}((F(y, z w)) F(x, y z w)} \\
& \quad \stackrel{(3.7)}{=} \lambda_{\rho(x) \rho(y)}(F(z, w) i(c(x, y, z w) F(x, y) F(x y, z w) \\
& \quad=i(c(x, y, z w) \underbrace{\lambda_{\beta(F(x, y)) \rho(x y)}(F(z, w))} F(x, y) F(x y, z w) \\
& \quad \stackrel{(\mathrm{CM} 3)}{=} i\left(c(x, y, z w) F(x, y) \lambda_{\rho(x y)}(F(z, w)) F(x, y)^{-1} F(x, y) F(x y, z w)\right. \\
& \quad=i(c(x, y, z w) F(x, y) \underbrace{(3.7)}_{\lambda_{\rho(x y)}(F(z, w)) F(x y, z w)} i(c(x, y, z w) F(x, y) i(c(x y, z, w)) F(x y, z) F(x y z, w) \\
& \quad=i(c(x, y, z w)) i(c(x y, z, w)) F(x, y) F(x y, z) F(x y z, w) .
\end{aligned}
$$

Cancelling the terms involving $F$, we have

$$
\lambda_{\rho(x)}(i(c(y, z, w)) i(c(x, y, z)) i(c(x, y z, w))=i(c(x, y, z w)) i(c(x y, z, w))
$$

and that means

$$
c \in Z^{3}(T, A) .
$$

With another choice of lifting for $f: \beta\left(F^{\prime}(x, y)\right)=f(x, y)$, there exists a function $u: T \times T \rightarrow i(A)$ such that $F^{\prime}(x, y)=u(x, y) F(x, y)$, with $u(x, y) \in N_{\rho(x y) \rho(x y)^{-1}}$, and im $u \subseteq i(A) \subseteq C(N)$.

We have (3.7) for $F^{\prime}$ for a new 3-cocycle $c^{\prime}$ :

$$
\lambda_{\rho(x)}\left(F^{\prime}(y, z)\right) F^{\prime}(x, y z)=i\left(c^{\prime}(x, y, z)\right) F^{\prime}(x, y) F^{\prime}(x y, z) .
$$


Since $F^{\prime}=u F$ :

$$
\lambda_{\rho(x)}(u(y, z) F(y, z)) u(x, y z) F(x, y z)=i\left(c^{\prime}(x, y, z)\right) u(x, y) F(x, y) u(x y, z) F(x y, z)
$$

since $\lambda$ is a homomorphism:

$$
\lambda_{\rho(x)}(u(y, z)) \lambda_{\rho(x)}(F(y, z)) u(x, y z) F(x, y z)=i\left(c^{\prime}(x, y, z)\right) u(x, y) F(x, y) u(x y, z) F(x y, z),
$$

since $\operatorname{im} u \subseteq C(N)$ :

$$
\delta^{2} u(x, y, z) \underbrace{\lambda_{\rho(x)}(F(y, z) F(x, y z)}=i\left(c^{\prime}(x, y, z)\right) F(x, y) F(x y, z),
$$

using (3.7) on the bracketed elements:

$$
\delta^{2} u(x, y, z) i(c(x, y, z)) F(x, y) F(x y, z)=i\left(c^{\prime}(x, y, z)\right) F(x, y) F(x y, z)
$$

and, cancelling the terms involving $F$ :

$$
\delta^{2} u(x, y, z) i(c(x, y, z))=i\left(c^{\prime}(x, y, z)\right)
$$

Thus, the new 3 -cocycle $c^{\prime}$ is cohomologous to $c$.

- If we choose another function $\rho^{\prime}: T \rightarrow S$ such that $\pi \circ \rho^{\prime}=\operatorname{id}_{T}$, we get $\left(\rho(x), \rho^{\prime}(x)\right) \in \operatorname{ker} \pi$ and there exists a function $v: T \rightarrow \beta(N)$ such that $\rho^{\prime}(x)=v(x) \rho(x)$, with $v(x) \in \beta(N)_{\rho(x) \rho(x)^{-1}}$.

$$
\begin{aligned}
\rho^{\prime}(x) & =v(x) \rho(x) \\
v(x)^{-1} \rho^{\prime}(x) & =v(x)^{-1} v(x) \rho(x) \\
v(x)^{-1} \rho^{\prime}(x) & =\rho(x) \rho(x)^{-1} \rho(x)=\rho(x)
\end{aligned}
$$

For this $\rho^{\prime}$, we have:

$$
\begin{aligned}
\rho^{\prime}(x) \rho^{\prime}(y) & =v(x) \rho(x) v(y) \rho(y) \\
& =v(x) \rho(x)\left(\rho(x)^{-1} \rho(x)\right) v(y) \rho(y) \\
& =v(x) \rho(x) v(y)\left(\rho(x)^{-1} \rho(x)\right) \rho(y) \\
& =v(x) \rho(x) v(y) \rho(x)^{-1} f(x, y) \rho(x y) \\
& =\underbrace{v(x) \rho(x) v(y) \rho(x)^{-1} f(x, y) v(x y)^{-1}} \rho^{\prime}(x y)
\end{aligned}
$$

and we can choose the bracketed expression as $f^{\prime}$ and write:

$$
f^{\prime}(x, y) v(x y)=v(x) \rho(x) v(y) \rho(x)^{-1} f(x, y)
$$

So we have:

$$
\begin{aligned}
f^{\prime}(x, y) v(x y) & =v(x) \rho(x) v(y) \rho(x)^{-1} f(x, y) \\
& =\rho^{\prime}(x) v(y) \rho^{\prime}(x)^{-1} v(x) f(x, y) .
\end{aligned}
$$


Again, we can lift this $f^{\prime}$ to a function $F^{\prime}: T \times T \rightarrow N$ such that $\beta\left(F^{\prime}(x, y)\right)=f^{\prime}(x, y)$.

Now, since $v: T \rightarrow \beta(N)$, there exists a function $V: T \rightarrow N$ such that $\beta(V(x))=v(x)$, and (3.9) becomes:

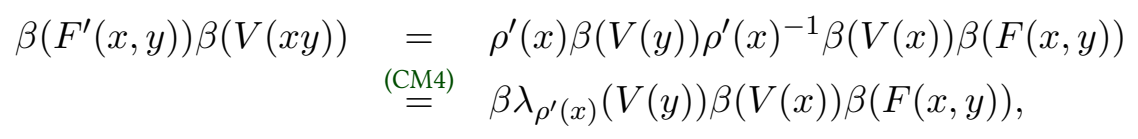

which implies $\left(F^{\prime}(x, y) V(x y), \lambda_{\rho^{\prime}(x)}(V(y)) V(x) F(x, y)\right) \in \operatorname{ker} \beta=i(A) \subseteq C(N)$ and this implies that there exists a function $w: T \times T \rightarrow A$ such that

$$
F^{\prime}(x, y) V(x y)=i(w(x, y)) \lambda_{\rho^{\prime}(x)}(V(y)) V(x) F(x, y) .
$$

$$
\begin{aligned}
& \lambda_{\rho^{\prime}(x)}\left(F^{\prime}(y, z)\right) \underbrace{F^{\prime}(x, y z) V(x y z)}=\lambda_{\rho^{\prime}(x)}\left(F^{\prime}(y, z)\right) i(w(x, y z)) \lambda_{\rho^{\prime}(x)}(V(y z)) V(x) F(x, y z) \\
& =i(w(x, y z)) \lambda_{\rho^{\prime}(x)}(\underbrace{F^{\prime}(y, z) V(y z)}) V(x) F(x, y z) \\
& =i(w(x, y z)) \lambda_{\rho^{\prime}(x)}\left(\left(i(w(y, z))\left(\lambda_{\rho^{\prime}(y)}(V(z)) V(y) F(y, z)\right) V(x) F(x, y z)\right.\right. \\
& =i(w(x, y z)) \lambda_{\rho^{\prime}(x)}(i(w(y, z)))\left(\lambda _ { \rho ^ { \prime } ( x ) } \left((\lambda_{\rho^{\prime}(y)}(V(z)) \lambda_{\rho^{\prime}(x)}(V(y)) \underbrace{\lambda_{\rho^{\prime}(x)}(F(y, z))} V(x) F(x, y z)\right.\right. \\
& =i(w(x, y z)) \lambda_{\rho^{\prime}(x)}\left(i ( w ( y , z ) ) \lambda _ { \rho ^ { \prime } ( x ) \rho ^ { \prime } ( y ) } ( V ( z ) ) \lambda _ { \rho ^ { \prime } ( x ) } ( V ( y ) ) \beta ( V ( x ) ) \left(\lambda_{\rho(x)}(F(y, z)) V(x) F(x, y z)\right.\right. \\
& =i(w(x, y z)) \lambda_{\rho^{\prime}(x)}\left(i ( w ( y , z ) ) \lambda _ { \rho ^ { \prime } ( x ) \rho ^ { \prime } ( y ) } ( V ( z ) ) \lambda _ { \rho ^ { \prime } ( x ) } ( V ( y ) ) V ( x ) \left(\lambda_{\rho(x)}(F(y, z)) V(x)^{-1} V(x) F(x, y z)\right.\right. \\
& =i(w(x, y z)) \lambda_{\rho^{\prime}(x)}(i(w(y, z)) \lambda_{\rho^{\prime}(x) \rho^{\prime}(y)}(V(z)) \lambda_{\rho^{\prime}(x)}(V(y)) V(x) \underbrace{\left(\lambda_{\rho(x)}(F(y, z)) F(x, y z)\right.} \\
& =i(w(x, y z)) \lambda_{\rho^{\prime}(x)}\left(i(w(y, z)) \lambda_{\rho^{\prime}(x) \rho^{\prime}(y)}(V(z)) \lambda_{\rho^{\prime}(x)}(V(y)) V(x) i(c(x, y, z)) F(x, y) F(x y, z)\right. \\
& =i(c(x, y, z)) i(w(x, y z)) \lambda_{\rho^{\prime}(x)}(i(w(y, z))) \\
& \lambda_{\rho^{\prime}(x) \rho^{\prime}(y)}(V(z)) \underbrace{\lambda_{\rho^{\prime}(x)}(V(y)) V(x) F(x, y)} F(x y, z) \\
& =i(c(x, y, z)) i(w(x, y z)) \lambda_{\rho^{\prime}(x)}(i(w(y, z))) \\
& \underbrace{\lambda_{\rho^{\prime}(x) \rho^{\prime}(y)}(V(z))} i(w(x, y))^{-1} F^{\prime}(x, y) V(x y) F(x y, z) \\
& =i(c(x, y, z)) i(w(x, y z)) \lambda_{\rho^{\prime}(x)}(i(w(y, z))) i(w(x, y))^{-1} \\
& \beta\left(F^{\prime}(x, y)\right) \lambda_{\rho^{\prime}(x y)}(V(z)) F^{\prime}(x, y) V(x y) F(x y, z) \\
& =i(c(x, y, z)) i(w(x, y z)) \lambda_{\rho^{\prime}(x)}(i(w(y, z))) i(w(x, y))^{-1} \\
& F^{\prime}(x, y) \lambda_{\rho^{\prime}(x y)}(V(z)) F^{\prime}(x, y)^{-1} F^{\prime}(x, y) V(x y) F(x y, z) \\
& =i(c(x, y, z)) i(w(x, y z)) \lambda_{\rho^{\prime}(x)}(i(w(y, z))) i(w(x, y))^{-1} \\
& F^{\prime}(x, y) \underbrace{\lambda_{\rho^{\prime}(x y)}(V(z)) V(x y) F(x y, z)} \\
& =i(c(x, y, z)) i(w(x, y z)) \lambda_{\rho^{\prime}(x)}(i(w(y, z))) i(w(x, y))^{-1} \\
& F^{\prime}(x, y) i(w(x y, z))^{-1} F^{\prime}(x y, z) V(x y z) \\
& =i(c(x, y, z)) i(w(x, y z)) \lambda_{\rho^{\prime}(x)}(i(w(y, z))) i(w(x, y))^{-1} i(w(x y, z))^{-1} \\
& F^{\prime}(x, y) F^{\prime}(x y, z) V(x y z)
\end{aligned}
$$

Cancelling the term $V(x y z)$ and noting that we have (3.7) for the pair $\left(\rho^{\prime}, F^{\prime}\right)$, we have

$$
i\left(c^{\prime}(x, y, z)\right)=i(c(x, y, z)) \delta^{2} i(w(x, y, z))
$$

Thus, we have shown that given a 4-term exact sequence like (3.1) we obtain a 3-cocycle whose cohomology class is independent of the possible choices.

Theorem 3.1.12. There is a well-defined function from $\mathcal{E}(T, A)$ to $H^{3}(T, A)$. 
Proof: By Proposition 3.1.11, it remains to show that equivalent sequences like (3.1) generate the same 3cocycle in $H^{3}(T, A)$.

Starting with equivalent sequences

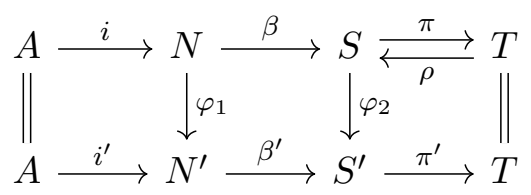

we do the whole procedure from Proposition 3.1.11 to find the 3-cocycle determined by the top sequence, obtaining $\rho: T \rightarrow S, f: T \times T \rightarrow \beta(N), F: T \times T \rightarrow N$, and $c: T \times T \times T \rightarrow A$, satisfying (CM3) and (CM4). And since the cohomology class of the cocycle determined by the crossed module sequence is independent of the choices, we choose $\rho^{\prime}: T \rightarrow S^{\prime}$ to be $\rho^{\prime}:=\varphi_{2} \circ \rho$.

Since $\pi \circ \rho=\operatorname{id}_{T} \Longrightarrow \pi^{\prime} \circ \varphi_{2} \circ \rho=\operatorname{id}_{T}$, we have $\pi^{\prime} \circ \rho^{\prime}=\mathrm{id}_{T}$. Also, by

$$
\begin{aligned}
\rho^{\prime}(x) \rho^{\prime}(y) & =\varphi_{2}(\rho(x)) \varphi_{2}(\rho(y)) \\
& =\varphi_{2}(f(x, y) \rho(x y)) \\
& =\varphi_{2}(f(x, y)) \varphi_{2}(\rho(x y)) \\
& =\varphi_{2}(f(x, y)) \rho^{\prime}(x y),
\end{aligned}
$$

we choose $f^{\prime}(x, y):=\varphi_{2}(f(x, y))$ to be the function satisfying (3.4) for $\rho^{\prime}$.

Applying $\varphi_{1}$ to (3.7) for the top sequence: $\varphi_{1}(\rho(x) \cdot F(x, z) F(x, y z))=\varphi_{1}(i(c(x, y, z)) F(x, y) F(x y, z))$. Using (3.3) on the left-hand side: $\varphi_{1}(\rho(x) \cdot F(y, z)) \varphi_{1}(F(x, y z))=\varphi_{2}(\rho(x)) \cdot \varphi_{1}(F(y, z)) \varphi_{1}(F(x, y z))$.

On the right-hand side, we use the commutativity of the diagram:

$\varphi_{1}(i(c(x, y, z))) \varphi_{1}(F(x, y)) \varphi_{1}(F(x y, z))=i^{\prime}(c(x, y, z)) \varphi_{1}(F(x, y)) \varphi_{1}(F(x y, z))$.

So we choose $F^{\prime}(x, y):=\varphi_{1}(F(x, y))$, which in fact lifts $f^{\prime}$ to $N$ :

$$
\begin{aligned}
\beta^{\prime}\left(F^{\prime}(x, y)\right) & =\beta^{\prime}\left(\varphi_{1}(F(x, y))\right) \\
& =\varphi_{2}(\beta(F(x, y))) \\
& =\varphi_{2}(f(x, y)) \\
& =f^{\prime}(x, y) .
\end{aligned}
$$

Therefore, equivalent sequences determine the same 3-cocycle $c$.

\subsubsection{Specifications on transversals and cocycles}

In this section, we prove some properties of the functions $\rho, f, F, c$ obtained from an element of $\mathcal{E}(T, A)$.

Consider sequences in $\mathcal{E}(T, A)$

$$
A \stackrel{i}{\rightarrow} N \stackrel{\beta}{\rightarrow} S \stackrel{\pi}{\rightarrow} T
$$


with $S$ a semilattice of groups (this will imply that $T$ is also a semilattice of groups) with transversal $\rho: T \rightarrow S$ of $\pi$ such that

$$
\rho(e) \rho(x)=\rho(e x)
$$

for all $e \in E(T), x \in T$ and transversal $\rho^{\prime}: \beta(N) \rightarrow N$ of $\beta$ such that

$$
\rho^{\prime}(e) \rho^{\prime}(s)=\rho^{\prime}(e s),
$$

for all $e \in E(S), s \in S$.

Lemma 3.1.13. The image of an idempotent element of $T$ under $\rho$ is an idempotent of $S$.

Proof: By definition, $\rho$ is such that $\pi \circ \rho=\mathrm{id}_{T}$. Then, for $e \in E(T)$ :

$$
\pi(\rho(e) \rho(e))=e=\pi(\rho(e)) .
$$

Since $\pi$ is an idempotent-separating homomorphism, this implies

$$
\rho(e) \rho(e)=\rho(e) .
$$

Lemma 3.1.14. The function $f: T \times T \rightarrow \beta(N)$ defined by (3.4) is such that, for all $x, y \in T, e \in E(T)$ :

(a)

$$
f(x, e y)=\rho(e) f(x, y)=f(e x, y) .
$$

(b)

$$
f(e, y)=\rho\left(e y y^{-1}\right), \quad f(x, e)=\rho\left(e x x^{-1}\right)
$$

Proof:

(a) Multiplying (3.4) by $\rho(e)$, we get:

$$
\rho(e) \rho(x) \rho(y)=\rho(e) f(x, y) \rho(x y) .
$$

Since $\rho(e)$ is an idempotent in $S$, we have

$$
\rho(e) \rho(x) \rho(y)=\rho(e) f(x, y) \rho(e) \rho(x y) .
$$

By (3.11),

$$
\rho(e x) \rho(y)=\rho(e) f(x, y) \rho(e x y) .
$$

Equation (3.4) for the left-hand side of this is

$$
\rho(e x) \rho(y)=f(e x, y) \rho(e x y) .
$$

This means

$$
\rho(e) f(x, y) \rho(e x y)=f(e x, y) \rho(e x y) .
$$




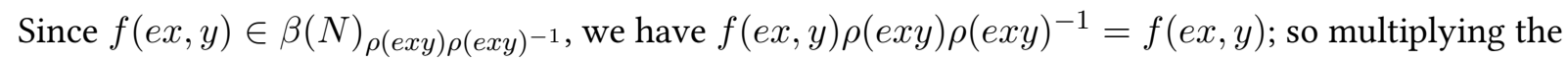
last equality by $\rho(e x y)^{-1}$ we get:

$$
\begin{aligned}
f(e x, y) & =\rho(e) f(x, y) \rho(e x y) \rho(e x y)^{-1} \\
& =\rho(e) f(x, y) \rho(e) \rho(x y) \rho(x y)^{-1} \\
& =\rho(e) f(x, y) .
\end{aligned}
$$

The other equality is similar.

(b) Equation (3.4) for the pair $(e, y)$ is

$$
\rho(e) \rho(y)=f(e, y) \rho(e y)
$$

multiplying this by $\rho(e y)^{-1}$ we get

$$
\rho(e) \rho(y) \rho(e y)^{-1}=f(e, y)
$$

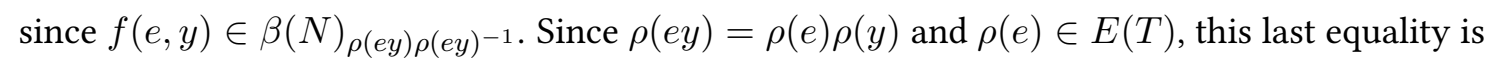

$$
f(e, y)=\rho(e) \rho(y) \rho(y)^{-1} .
$$

And since $\pi \circ \rho=\mathrm{id}_{T}$, with both maps isomorphims on idempotents, we get $\rho(e) \rho(y) \rho(y)^{-1}=$ $\rho\left(e y y^{-1}\right)$ and finally

$$
f(e, y)=\rho\left(e y y^{-1}\right)
$$

The other equality is similar.

Lemma 3.1.15. The function $F: T \times T \rightarrow N$ defined by (3.6) is such that, for all $x, y \in T, e \in E(T)$ :

(a)

$$
F(x, e y)=\alpha(\rho(e)) F(x, y)=F(e x, y)
$$

(b)

$$
F(e, y)=\alpha\left(\rho\left(e y y^{-1}\right)\right), \quad F(x, e)=\alpha\left(\rho\left(e x x^{-1}\right)\right)
$$

Proof:

(a) This follows from the definition of $F$, (3.13), and the fact that $\left.\beta\right|_{E(N)}=\alpha^{-1}$.

(b) Taking (3.6) for the pair $(e, y)$ we get

$$
\beta(F(e, y))=f(e, y)=\rho\left(e y y^{-1}\right)
$$

by the last lemma. And this implies

$$
F(e, y)=\alpha\left(\rho\left(e y y^{-1}\right)\right)
$$

since $\left.\beta\right|_{E(N)}=\alpha^{-1}$. 
The other equality is similar.

Lemma 3.1.16. The function $c: T \times T \times T \rightarrow A$ defined by (3.7) is such that, for all $x, y, z \in T, e \in E(T)$ :

(a)

$$
\theta(e) c(x, y, z)=c(e x, y, z)=c(x, e y, z)=c(x, y, e z)
$$

(b) is normalized, meaning

$$
\begin{aligned}
& c(e, y, z)=\theta\left(e y y^{-1} z z^{-1}\right), \\
& c(x, e, z)=\theta\left(e x x^{-1} z z^{-1}\right), \\
& c(x, y, e)=\theta\left(e x x^{-1} y y^{-1}\right) .
\end{aligned}
$$

Proof:

(a) The equality for both the triple $(x, e y, z)$ and $(x, y, e z)$ is similar, so we prove only for $(x, e y, z)$.

Also, since we have isomorphisms on all sets of idempotents, we have $i(\theta(e))=\alpha(\rho(e))$, for all $e \in$ $E(T)$.

Equation (3.7) for the triple $(x, e y, z)$ is

$$
\left.i(c(x, e y, z)) F(x, e y) F(x e y, z)=\lambda_{\rho(x)}(F(e y, z))\right) F(x, e y z) .
$$

Now, multiplying (3.7) by $\alpha(\rho(e))$ :

$$
\begin{gathered}
\left.\alpha(\rho(e)) i(c(x, y, z)) F(x, y) F(x y, z)=\alpha(\rho(e)) \lambda_{\rho(x)}(F(y, z))\right) F(x, y z) \\
\left.\alpha(\rho(e)) i(c(x, y, z)) F(x, e y) F(x e y, z)=\alpha(\rho(e)) \lambda_{\rho(x)}(F(y, z))\right) F(x, e y z) .
\end{gathered}
$$

The right-hand side of which is:

$$
\begin{aligned}
\left.\alpha(\rho(e)) \lambda_{\rho(x)}(F(e y, z))\right) F(x, e y z) & =\alpha(\rho(e)) \alpha\left(\rho(x) \rho(x)^{-1}\right) \lambda_{\rho(x)}(F(y, z)) F(x, e y z) \\
& =\alpha\left(\rho(e) \rho(x) \rho(x)^{-1}\right) \lambda_{\rho(x)}(F(y, z)) F(x, e y z) \\
& =\alpha\left(\rho(x) \rho(e) \rho(x)^{-1}\right) \lambda_{\rho(x)}(F(y, z)) F(x, e y z) \\
& =\lambda_{\rho(x)}\left(\alpha(\rho(e)) \lambda_{\rho(x)}(F(y, z)) F(x, e y z)\right. \\
& =\lambda_{\rho(x)}(\alpha(\rho(e) F(y, z))) F(x, e y z) \\
& =\lambda_{\rho(x)}(F(e y, z)) F(x, e y z) ;
\end{aligned}
$$

implying

$$
\alpha(\rho(e)) i(c(x, y, z)) F(x, e y) F(x e y, z)=i(c(x, e y, z)) F(x, e y) F(x e y, z) .
$$

$$
\begin{aligned}
i(c(x, e y, z)) F(x, e y) F(x e y, z) & =\alpha(\rho(e)) i(c(x, y, z)) F(x, e y) F(x e y, z) & \cdot F(x e y, z)^{-1} \\
i(c(x, e y, z)) F(x, e y) F(x e y, z) F(x e y, z)^{-1} & =\alpha(\rho(e)) i(c(x, y, z)) F(x, e y) F(x e y, z) F(x e y, z)^{-1} & \\
i(c(x, e y, z)) F(x, e y) \alpha\left(\rho\left(e x y z z^{-1} y^{-1} x^{-1}\right)\right) & =\alpha(\rho(e)) i(c(x, y, z)) F(x, e y) \alpha\left(\rho\left(e x y z z^{-1} y^{-1} x^{-1}\right)\right) & \cdot F(x, e y)^{-1} \\
i(c(x, e y, z)) F(x, e y) F(x, e y)^{-1} & =\alpha(\rho(e)) i(c(x, y, z)) F(x, e y) F(x, e y)^{-1} &
\end{aligned}
$$




$$
\begin{aligned}
i(c(x, e y, z)) \alpha\left(\rho\left(\text { xeyy }^{-1} x^{-1}\right)\right) & =\alpha(\rho(e)) i(c(x, y, z)) \alpha\left(\rho\left(x y y^{-1} x^{-1}\right)\right) \\
i(c(x, e y, z)) & =\alpha(\rho(e)) i(c(x, y, z)) .
\end{aligned}
$$

Now, for the equality $\alpha(\rho(e)) c(x, y, z)=c(e x, y, z)$, equation (3.7) for the triple $(e x, y, z)$ is:

$$
\left.i(c(e x, y, z)) F(e x, y) F(e x y, z)=\lambda_{\rho(e x)}(F(y, z))\right) F(e x, y z) .
$$

And again, multiplying (3.7) by $\alpha(\rho(e))$ :

$$
\begin{gathered}
\left.\alpha(\rho(e)) i(c(x, y, z)) F(x, y) F(x y, z)=\alpha(\rho(e)) \lambda_{\rho(x)}(F(y, z))\right) F(x, y z) \\
\left.\alpha(\rho(e)) i(c(x, y, z)) F(e x, y) F(e x y, z)=\alpha(\rho(e)) \lambda_{\rho(x)}(F(y, z))\right) F(e x, y z) .
\end{gathered}
$$

The right-hand side of which is:

$$
\begin{aligned}
\left.\alpha(\rho(e)) \lambda_{\rho(x)}(F(y, z))\right) F(e x, y z) & \left.=\lambda_{\rho(e)}\left(\lambda_{\rho(x)}(F(y, z))\right)\right) F(e x, y z) \\
& =\lambda_{\rho(e) \rho(x)}(F(y, z)) F(e x, y z) \\
& =\lambda_{\rho(e x)}(F(y, z)) F(e x, y z)
\end{aligned}
$$

implying

$$
\alpha(\rho(e)) i(c(x, y, z)) F(e x, y) F(e x y, z)=i(c(e x, y, z)) F(e x, y) F(e x y, z),
$$

and analogous to the previous case we get

$$
\alpha(\rho(e)) i(c(x, y, z))=i(c(e x, y, z)) .
$$

(b) Equation (3.7) for the triple $(e, y, z)$ is:

$$
\lambda_{\rho(e)}(F(y, z)) F(e, y z)=i(c(e, y, z)) F(e, y) F(e y, z) .
$$

Using the previous lemmas and the properties of the module structure:

$$
\begin{aligned}
\alpha(\rho(e)) F(y, z) \alpha\left(\rho\left(e y y^{-1} z z^{-1}\right)\right. & =i(c(e, y, z)) \alpha\left(\rho\left(e y y^{-1}\right)\right) \alpha(\rho(e)) F(y, z) \\
\alpha\left(\rho\left(e y y^{-1} z z^{-1}\right)\right) F(y, z) & =i(c(e, y, z)) \alpha\left(\rho\left(e y y^{-1}\right)\right) F(y, z) \\
\alpha(\rho(e)) F(y, z) & =i(c(e, y, z)) F(y, z) .
\end{aligned}
$$

Multiplying this by $F(y, z)^{-1}$ :

$$
\alpha(\rho(e)) F(y, z) F(y, z)^{-1}=i(c(e, y, z)) F(y, z) F(y, z)^{-1} .
$$

And this equals

$$
\begin{aligned}
\alpha(\rho(e)) \alpha\left(\rho\left(y z z^{-1} y^{-1}\right)\right) & =i(c(e, y, z)) \alpha\left(\rho\left(y z z^{-1} y^{-1}\right)\right) \\
\alpha\left(\rho\left(e y y^{-1} z z^{-1}\right)\right) & =i(c(e, y, z)) .
\end{aligned}
$$


The other equalities are similar.

Condition (3.17) is equivalent to $c$ being order-preserving. Recall that $f \in C^{n}(T, A)$ is order-preserving if

$$
s_{1} \leqslant t_{1}, \ldots, s_{n} \leqslant t_{n} \Longrightarrow f\left(s_{1}, \ldots, s_{n}\right) \leqslant f\left(t_{1}, \ldots, t_{n}\right),
$$

this subset of $C^{n}(T, A)$ is denoted by $C_{\leqslant}^{n}(T, A)$.

Theorem 3.1.12 and Lemma 3.1.16 imply the next.

Corollary 3.1.17. There is a well-defined function from $\mathcal{E}(T, A)$ to $H_{\leqslant}^{3}(T, A)$.

Lemma 3.1.18. Let $c \in C^{n}\left(T^{1}, A^{1}\right), n \geqslant 1$. Then $c \in C_{\leqslant}^{n}\left(T^{1}, A^{1}\right)$ if and only if for all $x_{1}, \ldots, x_{n} \in T$ and $e \in E(T)$ one has

$$
c\left(e x_{1}, x_{2}, \ldots, x_{n}\right)=\cdots=c\left(x_{1}, \ldots, x_{n-1}, e x_{n}\right)=\theta(e) c\left(x_{1}, \ldots, x_{n}\right) .
$$

Proof: The "if" part. Assume (3.18) and let $x_{i} \leqslant y_{i}, i=1, \ldots, n$. Then there are $e_{i} \in E(T)$, such that $x_{i}=e_{i} y_{i}$, $i=1, \ldots, n$. It follows that

$$
c\left(x_{1}, \ldots, x_{n}\right)=c\left(e_{1} y_{1}, \ldots, e_{n} y_{n}\right)=\theta\left(e_{1} \ldots e_{n}\right) c\left(y_{1}, \ldots, y_{n}\right) \leqslant c\left(y_{1}, \ldots, y_{n}\right) .
$$

Thus, $c \in C_{\leqslant}^{n}\left(T^{1}, A^{1}\right)$.

The "only if" part. Let $c \in C_{\leqslant}^{n}\left(T^{1}, A^{1}\right)$. Then $c\left(e x_{1}, x_{2}, \ldots, x_{n}\right) \leqslant c\left(x_{1}, \ldots, x_{n}\right)$, so that

$$
\begin{aligned}
c\left(e x_{1}, x_{2}, \ldots, x_{n}\right) & =c\left(e x_{1}, x_{2}, \ldots, x_{n}\right) c\left(e x_{1}, x_{2}, \ldots, x_{n}\right)^{-1} c\left(x_{1}, \ldots, x_{n}\right) \\
& =\theta\left(e x_{1} \ldots x_{n} x_{n}^{-1} \ldots x_{1}^{-1}\right) c\left(x_{1}, \ldots, x_{n}\right) \\
& =\theta(e) c\left(x_{1}, \ldots, x_{n}\right) .
\end{aligned}
$$

Also, $c\left(x_{1}, e x_{2}, x_{3}, \ldots, x_{n}\right) \leqslant c\left(x_{1}, \ldots, x_{n}\right)$ implies

$$
\begin{aligned}
c\left(x_{1}, e x_{2}, x_{3}, \ldots, x_{n}\right) & =c\left(x_{1}, e x_{2}, x_{3}, \ldots, x_{n}\right) c\left(x_{1}, e x_{2}, x_{3}, \ldots, x_{n}\right)^{-1} c\left(x_{1}, \ldots, x_{n}\right) \\
& =\theta\left(x_{1} e x_{2} \ldots x_{n} x_{n}^{-1} \ldots x_{1}^{-1}\right) c\left(x_{1}, \ldots, x_{n}\right) \\
& =\theta\left(x_{1} x_{1}^{-1} x_{1} e x_{2} \ldots x_{n} x_{n}^{-1} \ldots x_{1}^{-1}\right) c\left(x_{1}, \ldots, x_{n}\right) \\
& =\theta\left(x_{1} e x_{1}^{-1} x_{1} x_{2} \ldots x_{n} x_{n}^{-1} \ldots x_{1}^{-1}\right) c\left(x_{1}, \ldots, x_{n}\right) \\
& =\theta\left(x_{1} e x_{1}^{-1}\right) c\left(x_{1}, \ldots, x_{n}\right) \\
& =\theta(e) c\left(x_{1}, \ldots, x_{n}\right),
\end{aligned}
$$

this last equality is here to emphasize that (3.18) only holds when $T$ is a semilattice of groups.

Similarly one proves the remaining equalities of (3.18).

We want to prove the following:

Proposition 3.1.19. Every 3-cocycle is cohomologous to normalized 3-cocycle. Every normalized 3-coboundary 
is the coboundary of a normalized 2-cochain.

The next results aim to that.

Lemma 3.1.20. Let $c \in Z_{\leqslant}^{3}\left(T^{1}, A^{1}\right)$. Then for any $t \in T$ and $e \in E(T)$ one has

$$
c(e, t, e)=\theta\left(e^{-1} t^{-1} .\right.
$$

Proof: Indeed, applying the 3-cocycle identity to the quadruple $(e, t, e, t)$ and using (3.18), we have

$$
\begin{aligned}
\theta\left(e t t^{-1}\right) & =\theta(e) c(t, e, t) c(e t, e, t)^{-1} c(e, t e, t) c(e, t, e t)^{-1} c(e, t, e) \\
& =\theta\left(e t t^{-1}\right) c(e, t, e) \\
& =c(e, t, e) .
\end{aligned}
$$

Lemma 3.1.21. Let $c \in Z_{\leqslant}^{3}\left(T^{1}, A^{1}\right)$. Then $c$ is normalized if and only if

$$
c(t, e, e)=c(e, e, t)=\theta\left(e^{-1} t^{-1}\right)
$$

for all $t \in T$.

Proof: The "only if" part is trivial. Let us prove the "if" part. Suppose that $c(t, e, e)=\theta\left(e t t^{-1}\right)$ for all $t \in T$. Then applying the 3 -cocycle identity to the quadruple $(x, y, e, e)$ and using (3.18), we obtain

$$
\begin{aligned}
\theta\left(e x y y^{-1} x^{-1}\right) & =\eta_{x}(c(y, e, e)) c(x y, e, e)^{-1} c(x, y e, e) c\left(x, y, e^{2}\right)^{-1} c(x, y, e) \\
& =\eta_{x}\left(\theta\left(e y y^{-1}\right)\right) \theta\left(e x y y^{-1} x^{-1}\right) \theta(e) c(x, y, e) \\
& =c(x, y, e)
\end{aligned}
$$

Similarly, assuming $c(e, e, t)=\theta\left(e t t^{-1}\right)$ for all $t \in T$ and applying the 3 -cocycle identity to $(e, e, x, y)$, we have

$$
\begin{aligned}
\theta\left(e x y y^{-1} x^{-1}\right) & =\eta_{e}(c(e, x, y)) c\left(e^{2}, x, y\right)^{-1} c(e, e x, y) c(e, e, x y)^{-1} c(e, e, x) \\
& =\theta(e) c(e, x, y) \theta\left(e x y y^{-1} x^{-1}\right) \theta\left(e x x^{-1}\right) \\
& =c(e, x, y)
\end{aligned}
$$

Finally, both of the equalities (3.20) and the 3-cocycle identity applied to $(x, e, e, y)$ give

$$
\begin{aligned}
\theta\left(e x y y^{-1} x^{-1}\right) & =\eta_{x}(c(e, e, y)) c(x e, e, y)^{-1} c\left(x, e^{2}, y\right) c(x, e, e y)^{-1} c(x, e, e) \\
& =\eta_{x}\left(\theta\left(e y y^{-1}\right)\right) \theta(e) c(x, e, y) \theta\left(e x x^{-1}\right) \\
& =c(x, e, y)
\end{aligned}
$$

Lemma 3.1.22. Let $c \in Z_{\leqslant}^{3}\left(T^{1}, A^{1}\right)$. Define

$$
d(x, y)=c\left(x x^{-1}, x, y\right)^{-1} c\left(x, y, y y^{-1}\right) .
$$


Then $d \in C_{\leqslant}^{2}\left(T^{1}, A^{1}\right)$ and for all $t \in T$ and $e \in E(T)$

$$
\begin{aligned}
& d(e, e t)=c(e, e, t)^{-1}, \\
& d(t e, e)=c(t, e, e) .
\end{aligned}
$$

Proof: Indeed, $d$ is order-preserving, since $c$ is. Moreover, using (3.18) and (3.19) we have

$$
\begin{aligned}
& d(e, e t)=c(e, e, e t)^{-1} c\left(e, e t, e t t^{-1}\right)=\theta(e) c(e, e, t)^{-1} \theta\left(e t t^{-1}\right) c(e, t, e)=c(e, e, t)^{-1} \\
& d(t e, e)=c\left(e t t^{-1}, t e, e\right)^{-1} c(t e, e, e)=\theta\left(e t t^{-1}\right) c(e, t, e)^{-1} \theta(e) c(t, e, e)=c(t, e, e)
\end{aligned}
$$

Lemma 3.1.23. Let $c \in Z_{\leqslant}^{3}\left(T^{1}, A^{1}\right)$ and $d \in C_{\leqslant}^{2}\left(T^{1}, A^{1}\right)$ be defined by means of (3.21). Then $\tilde{c}:=c \cdot \delta^{2} d$ is normalized.

Proof: Let $t \in T$ and $e \in E(T)$. Using (3.19), (3.22) and (3.23) we shall calculate

$$
\begin{aligned}
\left(\delta^{2} d\right)(t, e, e) & =\eta_{t}(d(e, e)) d(t e, e)^{-1} d\left(t, e^{2}\right) d(t, e)^{-1} \\
& =\eta_{t}(c(e, e, e)) c(t, e, e)^{-1} \\
& =c(t, e, e)^{-1}
\end{aligned}
$$

and

$$
\begin{aligned}
\left(\delta^{2} d\right)(e, e, t) & =\eta_{e}(d(e, t)) d\left(e^{2}, t\right)^{-1} d(e, e t) d(e, e)^{-1} \\
& =\theta\left(e t t^{-1}\right) c(e, e, t)^{-1} c(e, e, e) \\
& =c(e, e, t)^{-1}
\end{aligned}
$$

It follows that $\tilde{c}(e, e, t)=\tilde{c}(t, e, e)=\theta\left(e t t^{-1}\right)$, so $\tilde{c}$ is normalized by Lemma 3.1.21.

Lemma 3.1.24. Let $c=\delta^{2} d \in B_{\leqslant}^{3}\left(T^{1}, A^{1}\right)$ be normalized. Then for all $t \in T$ and $e \in E(T)$

$$
d(e, t) d(e, e)^{-1}=\eta_{t}(d(e, e)) d(t, e)^{-1}=\theta\left(e t t^{-1}\right) .
$$

Proof: Indeed, using (3.18) we have

$$
\begin{aligned}
\theta\left(e t t^{-1}\right) & =c(e, e, t)=\left(\delta^{2} d\right)(e, e, t) \\
& =\theta(e) d(e, t) d\left(e^{2}, t\right)^{-1} d(e, e t) d(e, e)^{-1} \\
& =d(e, t) d(e, e)^{-1}
\end{aligned}
$$

and similarly

$$
\begin{aligned}
\theta\left(e t t^{-1}\right) & =c(t, e, e)=\left(\delta^{2} d\right)(t, e, e) \\
& =\eta_{t}(d(e, e)) d(t e, e)^{-1} d\left(t, e^{2}\right) d(t, e)^{-1} \\
& =\eta_{t}(d(e, e)) d(t, e)^{-1}
\end{aligned}
$$


Lemma 3.1.25. Let $c \in B_{\leqslant}^{3}\left(T^{1}, A^{1}\right)$ be normalized. Then there is a normalized $\tilde{d} \in C_{\leqslant}^{2}\left(T^{1}, A^{1}\right)$ such that $c=\delta^{2} \tilde{d}$

Proof: If $c=\delta^{2} d$ for some $d \in C_{\leqslant}^{2}\left(T^{1}, A^{1}\right)$, then we define $\tilde{d}=d \cdot \delta^{1} u$, where $u(t)=d\left(t, t^{-1}\right)^{-1}$. We immediately have $u \in C_{\leqslant}^{1}\left(T^{1}, A^{1}\right)$ and $\delta^{2} \tilde{d}=\delta^{2} d=c$. It remains to show that $\tilde{d}$ is normalized. Indeed, using (3.18) and (3.24) we have

$$
\begin{aligned}
& \tilde{d}(e, t)=d(e, t)\left(\delta^{1} u\right)(e, t)=d(e, t) \theta(e) u(t) u(e t)^{-1} u(e)=d(e, t) d(e, e)^{-1}=\theta\left(e t t^{-1}\right) \\
& \tilde{d}(t, e)=d(t, e)\left(\delta^{1} u\right)(t, e)=d(t, e) \eta_{t}(u(e)) u(t e)^{-1} u(t)=d(t, e) \eta_{t}\left(d(e, e)^{-1}\right)=\theta\left(e^{-1} t^{-1}\right) .
\end{aligned}
$$

Thus, Proposition 3.1 .19 is proved.

\subsection{From $H_{\leqslant}^{3}(T, A)$ to equivalence classes of crossed module extensions}

The goal of this section is to determine a bijective correspondence between $H_{\leqslant}^{3}(T, A)$ and $\mathcal{E}(T, A)$, with $T$ being a semilattice of groups.

So, for this section, $T$ will be a semilattice of groups and $A$ a Lausch's $T$-module with structure $(\eta, \theta)$, with $\eta: T \rightarrow$ end $A$ a homomorphism and $\theta: E(T) \rightarrow E(A)$ an isomorphism, as in Definition 3.1.3. Fixing notations, let $T=\bigsqcup_{e \in E(T)} T_{e}$ with each $T_{e}$ a group with identity element $e \in E(T)$, and since we have an isomorphism between idempotents of $T$ and $A$, we denote the semilattice of groups $A$ with the same symbols $e: A=\bigsqcup A_{e}$; this will mean that each group $A_{e}$ has identity $\theta(e): A_{e}=\left\{a \in A \mid a a^{-1}=a^{-1} a=\theta(e)\right\}$.

We shall prove the following:

Theorem 3.2.1. Any element in $H_{\leqslant}^{3}(T, A)$ determines a sequence like (3.1) in such a way that there is a welldefined function from $H_{\leqslant}^{3}(T, A)$ to $\mathcal{E}(T, A)$.

The proof will be done by establishing a series of results.

By Proposition 3.1.19, we represent any element of $H_{\leqslant}^{3}(T, A)$ by a $E$-normalized 3-cocycle; that is, a 3-cocycle that is normalized:

$$
\begin{aligned}
& c(e, y, z)=\theta\left(e y y^{-1} z z^{-1}\right), \\
& c(x, e, z)=\theta\left(e x x^{-1} z z^{-1}\right), \\
& c(x, y, e)=\theta\left(e x x^{-1} y y^{-1}\right) .
\end{aligned}
$$

and satisfies the condition equivalent to the order-preserving one:

$$
\theta(e) c(x, y, z)=c(e x, y, z)=c(x, e y, z)=c(x, y, e z)
$$

For this proof, we repeat the procedure for the group case: for each $e \in E(T)$, define the free group $F_{e}$ generated by the symbols $[x]_{e}, x \in T_{e}, x \neq e$, and denote the empty word of the group $F_{e}$ by $[e]$. 
Lemma 3.2.2. The union $S^{\prime}:=\bigsqcup F_{e}$ is a semilattice of groups.

Proof: For the union $S^{\prime}=\bigsqcup F_{e}$ we have the homomorphisms $\phi_{e, f}: F_{e} \rightarrow F_{e f}$ defined by $\phi_{e, e f}[x]_{e}=[f x]_{e f}$, for $e, f \in E(T)$. And the product of two generators $[x]_{e} \in F_{e}$ and $[y]_{f} \in F_{f}$ is

$$
[x]_{e}[y]_{f}:=\phi_{e, e f}\left([x]_{e}\right) \phi_{f, e f}\left([y]_{f}\right)=[f x]_{e f}[e y]_{e f} \in F_{e f}
$$

Associativity: for $[x]_{e},[y]_{f},[z]_{g}$, with $e, f, g \in E(T)$ :

$$
\begin{aligned}
\left([x]_{e}[y]_{f}\right)[z]_{g} & =\left([f x]_{e f}[e y]_{e f}\right)[z]_{g} & {[x]_{e}\left([y]_{f}[z]_{g}\right) } & =[x]_{e}\left([g y]_{f g}[f z]_{f g}\right) \\
& =\left([g f x]_{e f g}[\text { gey }]_{e f g}\right)[\text { ef } z]_{e f g}, & & =[f g x]_{e f g}\left([\text { egy }]_{\text {efg }}[\text { ef } z]_{\text {efg }}\right),
\end{aligned}
$$

and these last products are equal, using the associativity of the group $F_{\text {efg }}$.

Remark 3.2.3. The product in $S^{\prime}=\bigsqcup F_{e}$ also holds for $[x]_{e}[f]=[f x]_{e f}$ and $[e][y]_{f}=[e y]_{e f}$.

For each group $F_{e}$, there is an epimorphism $\pi_{e}: F_{e} \rightarrow T_{e}$ determined by $\pi_{e}[x]_{e}=x$; the kernel of $\pi_{e}$ is also a free subgroup, denoted by $K_{e}$, generated by $[e] \neq[x, y]_{e}:=[x]_{e}[y]_{e}[x y]_{e}^{-1}$. More generally, the symbol $[x, y]_{e, f}$ denotes the element

$$
[x, y]_{e, f}:=[x]_{e}[y]_{f}[x y]_{e f}^{-1},
$$

and in particular, $[x, y]_{e}$ denotes $[x, y]_{e, e}$.

The following lemmas state some properties of the elements $[x, y]_{e, f}$.

Lemma 3.2.4. $[e][x, y]_{f}=[e x, y]_{e f, f}=[x, e y]_{f, e f}=[e x, e y]_{e f}$, if $e \neq x y y^{-1} x^{-1}$.

Proof:

$$
\begin{array}{rlrl}
{[e][x, y]_{f}} & =[e][x]_{f}[y]_{f}[x y]_{f}^{-1} & \\
=[e][x]_{f}[y]_{f}[x y]_{f}^{-1}[e]^{-1} & =[x]_{f}[e][y]_{f}[x y]_{f}^{-1}[e]^{-1} \\
=[e x]_{e f}[y]_{f}[e x y]_{e f}^{-1} & =[x]_{f}[e y]_{e f}[x e y]_{e f}^{-1} \\
=[e x, y]_{e f, f} & =[x]_{f, e f} \\
{[e][x, y]_{f}} & =[e][x]_{f}[y]_{f}[x y]_{f}^{-1} \\
& =[e][x]_{f}[e][y]_{f}[e][x y]_{f}^{-1} \\
& =[e x]_{e f}[e y]_{e f}[e x y]_{e f}^{-1} \\
& =[e x, e y]_{e f} .
\end{array}
$$

Lemma 3.2.5. $[x, y]_{e, f}=[f x, e y]_{e f}$.

Proof:

$$
[x, y]_{e, f}=[x]_{e}[y]_{f}[x y]_{e f}^{-1}
$$




$$
\begin{aligned}
& =[f x]_{e f}[e y]_{e f}[e f x y]_{e f}^{-1} \\
& =[f x, e y]_{e f} .
\end{aligned}
$$

Lemma 3.2.6. $[x, f]_{e, f}=[e f]=[e, y]_{e, f}$.

Proof:

$$
\begin{aligned}
{[x, f]_{e, f} } & =[x]_{e}[f][x f]_{e f}^{-1} & {[e, y]_{e, f} } & =[e][y]_{f}[e y]_{e f}^{-1} \\
& =[f x]_{e f}[f x]_{e f}^{-1} & & =[e y]_{e f}[e y]_{e f}^{-1} \\
& =[e f], & & =[e f] .
\end{aligned}
$$

We define the direct product $N_{e}^{\prime}:=A_{e} \times K_{e}$ and, taking elements $(a, k)_{e}$ of $N_{e}^{\prime}$ and $(b, l)_{f}$ of $N_{f}^{\prime}$ their product will be

$$
(a, k)_{e}(b, l)_{f}:=(a b,[f] k \cdot[e] l)_{e f} .
$$

With this, the union of groups $N^{\prime}:=\bigsqcup N_{e}^{\prime}$ is a semilattice of groups.

For each $x \in T_{e}$, define the function $\lambda_{x, f}^{\prime}: K_{f} \rightarrow N_{e f}^{\prime}$ that acts on a free generator of $[y, z]_{f}$ of $K_{f}$ as follows:

$$
\lambda_{x, f}^{\prime}\left([y, z]_{f}\right):=\left(c(x, y, z),[x, y]_{e, f}[x y, z]_{e f, f}[x, y z]_{e, f}^{-1}\right) .
$$

The element $c(x, y, z)$ belongs to $A_{e f}$, since $c(x, y, z) \in A_{x y z z^{-1} y^{-1} x^{-1}}=A_{e f}$, for $y, z \in T_{f}$ and $x \in T_{e}$; as well as the product $[x, y]_{e, f}[x y, z]_{e f, f}[x, y z]_{e, f}^{-1}$ in fact belongs to $K_{e f}$ :

$$
\begin{aligned}
& {[x, y]_{e, f}=[x]_{e}[y]_{f}[x y]_{e f}^{-1} \quad[x y, z]_{e f, f}=[x y]_{e f}[z]_{f}[x y z]_{e f}^{-1} \quad[x, y z]_{e, f}=[x]_{e}[y z]_{f}[x y z]_{e f}^{-1}} \\
& =[f x]_{e f}[e y]_{e f}[x y]_{e f}^{-1}, \quad=[x y]_{e f}[e z]_{e f}[x y z]_{e f}^{-1}, \quad=[f x]_{e f}[y z]_{e f}[x y z]_{e f}^{-1} .
\end{aligned}
$$

So, the codomain of $\lambda_{x, f}^{\prime}$ really is $N_{e f}^{\prime}$.

The case of the elements $[f, z]_{f}$ or $[y, f]_{f}$ are also defined, since the 3 -cocycle $c$ was taken $E$-normalized:

$$
\begin{aligned}
\lambda_{x, f}^{\prime}\left([f, z]_{f}\right) & \left.=\left(c(x, f, z),[x, f]_{e, f}[x f, z]_{e f, f}[x, f z]_{e, f}^{-1}\right]\right) \\
& =\left(\theta(e f),[f x, e f]_{e f}[f x, e z]_{e f}[f x, e z]_{e f}^{-1}\right) \\
& =\left(\theta(e f),[e f]_{e f}[f x, e z]_{e f}[f x, e z]_{e f}^{-1}\right), \\
& =\left(\theta(e f),[e f]_{e f}\right), \\
\lambda_{x, f}^{\prime}\left([y, f]_{f}\right) & =\left(c(x, y, f),[x, y]_{e, f}[x y, f]_{e f, f}[x, y f]_{e, f}^{-1}\right) \\
& =\left(\theta(e f),[f x, e y]_{e f}[x y, e f]_{e f}[f x, e y]_{e f}^{-1}\right) \\
& =\left(\theta(e f),[f x, e y]_{e f}[e f]_{e f}[f x, e y]_{e f}^{-1}\right) \\
& =\left(\theta(e f),[f x, e y]_{e f}[f x, e y]_{e f}^{-1}\right) \\
& =\left(\theta(e f),[e f]_{e f}\right) .
\end{aligned}
$$


Since each $K_{f}$ is a free group, this $\lambda_{x, f}^{\prime}$ can be extended to a homomorphism of groups $\lambda_{x, f}^{\prime}: K_{f} \rightarrow N_{e f}^{\prime}$, which will be called by the same name.

The special case of $x=e$ is

$$
\lambda_{e, f}^{\prime}\left([y, z]_{f}\right)=\left(\theta(e f),[e][y, z]_{f}\right)
$$

since

$$
\begin{aligned}
\lambda_{e, f}^{\prime}\left([y, z]_{f}\right) & =\left(c(e, y, z),[e, y]_{e, f}[e y, z]_{e f, f}[e, y z]_{e, f}^{-1}\right) \\
& =\left(\theta(e f),[e f][e][y, z]_{f}[e f]\right) \\
& =\left(\theta(e f),[e][y, z]_{f}\right)
\end{aligned}
$$

Now, for $x \in T_{e}$, we define $\lambda_{x, f}^{\prime}: N_{f}^{\prime} \rightarrow N_{e f}^{\prime}$ acting on a free generator $[y, z]_{f}$ of $N_{f}^{\prime}$ as:

$$
\lambda_{x, f}^{\prime}\left(a,[y, z]_{f}\right):=\left(\eta_{x}(a) c(x, y, z),[x, y]_{e, f}[x y, z]_{e f, f}[x, y z]_{e, f}^{-1}\right) .
$$

We want a homomorphism of semigroups $\lambda_{x}: N^{\prime} \rightarrow N^{\prime}$. So, taking $\left(a,[y, z]_{f}\right) \in N_{f}^{\prime}$ and $\left(b,[v, w]_{g}\right) \in N_{g}^{\prime}$, we want the following equality to hold:

$$
\lambda_{x, f}^{\prime}\left(a,[y, z]_{f}\right) \lambda_{x, g}^{\prime}\left(b,[v, w]_{g}\right)=\lambda_{x, f g}^{\prime}\left(a b,[y, z]_{f}[v, w]_{g}\right)
$$

We have:

$$
\begin{aligned}
& \lambda_{x, f}^{\prime}\left(a,[y, z]_{f}\right)=\left(\eta_{x}(a) c(x, y, z),[x, y]_{e, f}[x y, z]_{e f, f}[x, y z]_{e, f}^{-1}\right), \\
& \lambda_{x, g}^{\prime}\left(b,[v, w]_{g}\right)=\left(\eta_{x}(b) c(x, v, w),[x, v]_{e, g}[x v, w]_{e g, g}[x, v w]_{e, g}^{-1}\right)
\end{aligned}
$$

So, the left-hand side of (3.26) is

$$
\left(\eta_{x}(a) \eta_{x}(b) c(x, y, z) c(x, v, w),[x, y]_{e, f}[x y, z]_{e f, f}[x, y z]_{e, f}^{-1}[x, v]_{e, g}[x v, w]_{e g, g}[x, v w]_{e, g}^{-1}\right)
$$

Now for the right-hand side of (3.26):

$$
\begin{aligned}
\lambda_{x, f g}^{\prime}\left(a b,[y, z]_{f}[v, w]_{g}\right)= & \lambda_{x, f g}^{\prime}\left(a b,[g][y, z]_{f}[f][v, w]_{g}\right) \\
= & \lambda_{x, f g}^{\prime}\left(a b,[g y, g z]_{f g}[f v, f w]_{f g}\right) \\
= & \left(\eta_{x}(a b) c(x, g y, g z) c(x, f v, f w),\right. \\
& {\left.[x, g y]_{e, f g}[x g y, g z]_{e f g, f g}[x, g y z]_{e, f g}^{-1}[x, f v]_{e, f g}[x f v, f w]_{e f g, f g}[x, f v w]_{e, f g}^{-1}\right) } \\
= & \left(\eta_{x}(a b) \theta(g) c(x, y, z) \theta(f) c(x, v, w),\right. \\
& {\left.[g][x, y]_{e, f}[g][x y, z]_{e f, f}[g][x, y z]_{e, f}^{-1}[f][x, v]_{e, g}[f][x v, w]_{e g, g}[f][x, v w]_{e, f g}^{-1}\right) } \\
= & \left(\eta_{x}(a b) c(x, y, z) c(x, v, w),\right. \\
& {\left.[x, y]_{e, f}[x y, z]_{e f, f}[x, y z]_{e, f}^{-1}[x, v]_{e, g}[x v, w]_{e g, g}[x, v w]_{e, f g}^{-1}\right) . }
\end{aligned}
$$

Thus, the equation (3.26) holds. 
For $[x, y]_{e, f} \in K_{e f}$, define $\mu\left([x, y]_{e, f}\right): N^{\prime} \rightarrow N^{\prime}$ by

$$
\mu\left([x, y]_{e, f}\right)(a, k):=\left(\theta(e f) a,[x, y]_{e, f} k[x, y]_{e, f}^{-1}\right) .
$$

Lemma 3.2.7. $\mu([e])=\left.\mathrm{id}\right|_{e N^{\prime}}$.

Proof:

$$
\begin{aligned}
\mu([e])(a, k)_{f} & =\left(\theta(e) a,[e] k[e]^{-1}\right) \\
& =(\theta(e) a,[e] k)
\end{aligned}
$$

which is the element $(a, k)_{f}$ projected to the component $N_{e f}^{\prime} \subseteq e N^{\prime}$.

Claim: For $x \in T_{e}, y \in T_{f}$,

$$
\lambda_{x}^{\prime} \lambda_{y}^{\prime}=\mu\left([x, y]_{e, f}\right) \lambda_{x y}^{\prime} .
$$

It suffices to show that the endomorphism on each side has the same effect on any generator $[z, w]_{g}$ of $K_{g}$ :

$$
\begin{aligned}
\lambda_{x}^{\prime} \lambda_{y}^{\prime}\left(a,[z, w]_{g}\right)= & \lambda_{x}^{\prime}\left(\eta_{y}(a) c(y, z, w),[y, z]_{f, g}[y z, w]_{f g, g}[y, z w]_{f, g}^{-1}\right) \\
= & \left(\eta_{x}(a c(y, z, w)) c(x, y, z) c(x, y z, w) c(x, y, z w)^{-1}\right. \\
& {[x, y]_{e, f}[x y, z]_{e f, g}[x, y z]_{e, f g}^{-1}[x, y z]_{e, f g}[x y z, w]_{e f g, g}[x, y z w]_{e, f g}^{-1} } \\
& {\left.[x, y z w]_{e, f g}[x y, z w]_{e f, g}^{-1}[x, y]_{e, f}^{-1}\right) } \\
= & \left(\eta_{x}\left(\eta_{y}(a)\right) \eta_{x}(c(y, z, w)) c(x, y, z) c(x, y z, w) c(x, y, z w)^{-1}\right. \\
& {\left.[x, y]_{e, f}[x y, z]_{e f, g}[e f g][x y z, w]_{e f g, g}[e f g][x y, z w]_{e f, g}^{-1}[x, y]_{e, f}^{-1}\right), }
\end{aligned}
$$

this product of $c^{\prime}$ 's include all but $c(x y, z, w)^{-1}$ of $\delta^{3} c(x, y, z, w)$. This results in:

$$
\begin{aligned}
\lambda_{x}^{\prime} \lambda_{y}^{\prime}\left(a,[z, w]_{g}\right) & =\left(\eta_{x y}(a) c(x y, z, w),[x, y]_{e, f}[x y, z]_{e f, g}[x y z, w]_{e f g, g}[x y, z w]_{e f, g}^{-1}[x, y]_{e, f}^{-1}\right) \\
& =\mu\left([x, y]_{e, f}\right) \lambda_{x y}^{\prime}\left(a,[z, w]_{g}\right) .
\end{aligned}
$$

For $x \in T_{e}$, we have

$$
\left(\lambda_{x}^{\prime}\right)^{-1}=\mu\left(\left[x^{-1}, x\right]_{e}\right)^{-1} \circ \lambda_{x^{-1}}^{\prime}=\lambda_{x^{-1}}^{\prime} \circ \mu\left(\left[x, x^{-1}\right]_{e}\right)^{-1} .
$$

Using (3.27):

$$
\begin{aligned}
\left(\lambda_{x}^{\prime}\right)^{-1} \circ \lambda_{x}^{\prime} & =\mu\left(\left[x^{-1}, x\right]_{e}\right)^{-1} \circ \lambda_{x^{-1}}^{\prime} \circ \lambda_{x}^{\prime} \\
& =\mu\left(\left[x^{-1}, x\right]_{e}\right)^{-1} \circ \mu\left(\left[x^{-1}, x\right]_{e}\right) \circ \lambda_{x^{-1} x}^{\prime} \\
& =\mu\left(\left[x^{-1}, x\right]_{e}^{-1}\left[x^{-1}, x\right]_{e}\right) \circ \lambda_{e}^{\prime} \\
& =\mu[e] ; \\
\lambda_{x}^{\prime} \circ\left(\lambda_{x}^{\prime}\right)^{-1} & =\lambda_{x}^{\prime} \circ \lambda_{x^{-1}}^{\prime} \circ \mu\left(\left[x, x^{-1}\right]_{e}\right)^{-1} \\
& =\mu\left(\left[x, x^{-1}\right]_{e} \circ \lambda_{e}^{\prime} \circ \mu\left(\left[x, x^{-1}\right]_{e}\right)^{-1}\right. \\
& =\mu\left(\left[x, x^{-1}\right]_{e}\left[x, x^{-1}\right]_{e}^{-1}\right)
\end{aligned}
$$


$=\mu[e]$.

Since $\left.\mu[e]\right|_{e N^{\prime}}=\operatorname{id}_{e N^{\prime}}$, we also have $\lambda_{x}^{\prime} \in \operatorname{Aut}\left(e N^{\prime}\right)$, thus $\lambda_{x}^{\prime} \in$ end $N^{\prime}$.

The function $\lambda^{\prime}: T_{e} \rightarrow$ end $N^{\prime}$ can be extended to a unique well-defined homomorphism $\bar{\lambda}^{e}: F_{e} \rightarrow$ end $N^{\prime}$ such that $\bar{\lambda}_{[x]}^{e}=\lambda_{x}^{\prime}$.

Remark 3.2.8. $\bar{\lambda}_{[x]^{-1}}=\left(\lambda_{x}^{\prime}\right)^{-1}$.

Proposition 3.2.9. For the semilattices of groups $S^{\prime}=\bigsqcup F_{e}$ and $N^{\prime}=\bigsqcup N_{e}^{\prime}$, define $\lambda: S^{\prime} \rightarrow$ end $N^{\prime}$ by $\lambda_{s}:=\bar{\lambda}_{s}^{e}$, for $s \in F_{e}$. Then, $\lambda$ is a homomorphism.

Proof: Of course, we want to prove $\lambda_{s} \lambda_{t}=\lambda_{s t}$, for all $s \in F_{e}, t \in F_{f}$. The left-hand side of this equals $\bar{\lambda}_{s}^{e} \bar{\lambda}_{t}^{f}$, whilst the right-hand side of this equals $\bar{\lambda}_{[f] s \cdot[e] t}^{e f}$.

We start the proof with generators and move on from there.

CASE 1: For $s=[x]_{e}$ and $t=[y]_{f}$, we have $s t=[f x]_{e f}[e y]_{e f}$ and:

$$
\begin{array}{rlrl}
\lambda_{s t} & =\bar{\lambda}_{[f x]_{e f}[e y]_{e f}} & \lambda_{s} \lambda_{t}=\bar{\lambda}_{[x]}^{e} \bar{\lambda}_{[y]}^{f} \\
& =\bar{\lambda}_{[f x]_{e f}}^{e f} \bar{\lambda}_{[e y]_{e f}}^{e f} & & =\lambda_{x}^{\prime} \lambda_{y}^{\prime} \\
& =\lambda_{f x}^{\prime} \lambda_{e y}^{\prime} & \stackrel{(3.27)}{=} \mu\left([x, y]_{e, f}\right) \lambda_{x y}^{\prime} \\
& \stackrel{(3.27)}{=} \mu\left([f x, e y]_{e f}\right) \lambda_{e f x y}^{\prime}, &
\end{array}
$$

since $e f x y=x y$ in $T$, and by Lemma 3.2.5 we have $\lambda_{s} \lambda_{t}=\lambda_{s t}$.

CASE 2: For $s=[x]_{e}^{-1}$ and $t=[y]_{f}$, we have $s t=[f x]_{e f}^{-1}[e y]_{e f}$ and:

$$
\begin{array}{rlrl}
\lambda_{s t} & =\bar{\lambda}_{[f x]_{e f}^{-1}[e y]_{e f}} & \lambda_{s} \lambda_{t} & =\bar{\lambda}_{[x]^{-1}}^{e} \bar{\lambda}_{[y]}^{f} \\
& =\bar{\lambda}_{[f x]_{e f}^{-1}}^{-1} \bar{\lambda}_{[e y]_{e f}}^{f} & & =\left(\lambda_{x}^{\prime}\right)^{-1} \lambda_{y}^{\prime} \\
& =\left(\lambda_{f x}^{\prime}\right)^{-1} \lambda_{e y}^{\prime} & \stackrel{(3.28)}{=} \mu\left(\left[x^{-1}, x\right]_{e}\right)^{-1} \lambda_{x^{-1}}^{\prime} \lambda_{y}^{\prime} \\
& \stackrel{(3.28)}{=} \mu\left(\left[f x^{-1}, f x\right]_{e f}\right)^{-1} \lambda_{f x^{-1}}^{\prime} \lambda_{e y}^{\prime} & & =\mu\left(\left[x^{-1}, x\right]_{e}\right)^{-1} \mu\left(\left[x^{-1}, y\right]_{e, f}\right) \lambda_{x^{-1} y}^{\prime}, \\
& =\mu\left(\left[f x^{-1}, f x\right]_{e f}\right)^{-1} \mu\left(\left[f x^{-1}, e y\right]_{e f}\right) \lambda_{e f x^{-1} y}^{\prime}, &
\end{array}
$$

since $e$ f $x^{-1} y=x^{-1} y$ in $T$, and by Lemma 3.2.5 we have $\lambda_{s} \lambda_{t}=\lambda_{s t}$.

CASE 3: For $s=[x]_{e}$ and $t=[y]_{f}^{-1}$, we have $s t=[f x]_{e f}[e y]_{e f}^{-1}$ and:

$$
\begin{array}{rlrl}
\lambda_{s t} & =\bar{\lambda}_{[f x]_{e f}[e y]_{e f}^{-1}}^{e f} & \lambda_{s} \lambda_{t} & =\bar{\lambda}_{[x]}^{e} \bar{\lambda}_{[y]}^{f} \\
& =\bar{\lambda}_{[f x]_{e f}{ }^{-1}}^{e f} \bar{\lambda}_{[e y]_{e f}^{-1}}^{-1} & & =\lambda_{x}^{\prime}\left(\lambda_{y}^{\prime}\right)^{-1} \\
& =\lambda_{f x}^{\prime}\left(\lambda_{e y}^{\prime}\right)^{-1} & & =\lambda_{x}^{\prime} \lambda_{y^{-1}}^{\prime} \mu\left(\left[y, y^{-1}\right]_{f}\right)^{-1} \\
& =\lambda_{f x}^{\prime} \lambda_{e y^{-1}}^{\prime} \mu\left(\left[e y, e y^{-1}\right]_{e f}\right)^{-1} & & =\mu\left(\left[x, y^{-1}\right]_{e, f}\right) \lambda_{x y^{-1}}^{\prime} \mu\left(\left[y, y^{-1}\right]_{f}\right)^{-1}, \\
& =\mu\left(\left[f x, e y^{-1}\right]_{e f}\right) \lambda_{e f x y^{-1}}^{\prime} \mu\left(\left[e y, e y^{-1}\right]_{e f}\right)^{-1}, &
\end{array}
$$

and again we have $\lambda_{s} \lambda_{t}=\lambda_{s t}$. 
CASE 4: For $s=[x]_{e}^{-1}$ and $t=[y]_{f}^{-1}$, we have $s t=[f x]_{e f}^{-1}[e y]_{e f}^{-1}$ and:

$$
\begin{aligned}
\lambda_{s t} & =\bar{\lambda}_{[f x]_{e f}^{-1}[e y]_{e f}^{-1}}^{e f} \\
& =\bar{\lambda}_{[f x]_{e f}^{-f}}^{-1} \bar{\lambda}_{[e f]_{e f}^{-1}} \\
& =\left(\lambda_{f x}^{\prime}\right)^{-1}\left(\lambda_{e y}^{\prime}\right)^{-1} \\
& =\mu\left(\left[f x^{-1}, f x\right]_{e f}\right)^{-1} \lambda_{f x^{-1}}^{\prime} \lambda_{e y^{-1}}^{\prime} \mu\left(\left[e y, e y^{-1}\right]_{e f}\right)^{-1} \\
& =\mu\left(\left[f x^{-1}, f x\right]_{e f}\right)^{-1} \mu\left(\left[f x^{-1}, e y^{-1}\right]_{e f}\right) \lambda_{e f x^{-1} y^{-1}}^{\prime} \mu\left(\left[e y, e y^{-1}\right]_{e f}\right)^{-1}, \\
\lambda_{s} \lambda_{t} & =\bar{\lambda}_{[x]^{-1}}^{e} \bar{\lambda}_{[y]^{-1}}^{f} \\
& =\left(\lambda_{x}^{\prime}\right)^{-1}\left(\lambda_{y}^{\prime}\right)^{-1} \\
& =\mu\left(\left[x^{-1}, x\right]_{e}\right)^{-1} \lambda_{x^{-1}}^{\prime} \lambda_{y^{-1}}^{\prime} \mu\left(\left[y, y^{-1}\right]_{f}\right)^{-1} \\
& =\mu\left(\left[x^{-1}, x\right]_{e}\right)^{-1} \mu\left(\left[x^{-1}, y^{-1}\right]_{e, f}\right) \lambda_{x^{-1} y^{-1}}^{\prime} \mu\left(\left[y, y^{-1}\right]_{f}\right)^{-1},
\end{aligned}
$$

and we have $\lambda_{s} \lambda_{t}=\lambda_{s t}$.

With these four cases we proved, for $x \in T_{e}$ and $y \in T_{f}$ :

$$
\left(\lambda_{x}^{\prime}\right)^{\varepsilon}\left(\lambda_{y}^{\prime}\right)^{\nu}=\left(\lambda_{f x}^{\prime}\right)^{\varepsilon}\left(\lambda_{e y}^{\prime}\right)^{\nu}
$$

again with $\varepsilon, \nu=\{-1,+1\}$.

Arbitrary elements $s \in F_{e}$ and $t \in F_{f}$ are $s=\left[x_{1}\right]_{e}^{\varepsilon_{1}} \cdots\left[x_{n}\right]_{e}^{\varepsilon_{n}}$, with $\varepsilon_{i} \in\{-1,+1\}$, for $i=1, \ldots, n$, and $t=\left[y_{1}\right]_{f}^{\nu_{1}} \cdots\left[y_{m}\right]_{f}^{\nu_{m}}$, with $\nu_{j} \in\{-1,+1\}$, for $j=1, \ldots, m$. Then,

$$
\begin{aligned}
\lambda_{s} & =\bar{\lambda}_{\left[x_{1}\right]^{\varepsilon_{1}}}^{e} \cdots \bar{\lambda}_{\left[x_{n}\right]^{\varepsilon_{n}}}^{e} & \lambda_{t} & =\bar{\lambda}_{\left[y_{1}\right]^{\nu_{1}}}^{f} \cdots \bar{\lambda}_{\left[x_{m}\right]^{\nu_{m}}}^{f} \\
& =\left(\lambda_{x_{1}}^{\prime}\right)^{\varepsilon_{1}} \cdots\left(\lambda_{x_{n}}^{\prime}\right)^{\varepsilon_{n}}, & & =\left(\lambda_{y_{1}}^{\prime}\right)^{\nu_{1}} \cdots\left(\lambda_{y_{m}}^{\prime}\right)^{\nu_{m}} .
\end{aligned}
$$

Now,

$$
\begin{aligned}
& \lambda_{s} \lambda_{t}=\left(\lambda_{x_{1}}^{\prime}\right)^{\varepsilon_{1}} \cdots\left(\lambda_{x_{n}}^{\prime}\right)^{\varepsilon_{n}}\left(\lambda_{y_{1}}^{\prime}\right)^{\nu_{1}} \cdots\left(\lambda_{y_{m}}^{\prime}\right)^{\nu_{m}} \\
& =\left(\lambda_{x_{1}}^{\prime}\right)^{\varepsilon_{1}} \cdots\left(\lambda_{x_{n-1}}^{\prime}\right)^{\varepsilon_{n-1}}\left(\lambda_{f x_{n}}^{\prime}\right)^{\varepsilon_{n}}\left(\lambda_{e y_{1}}^{\prime}\right)^{\nu_{1}}\left(\lambda_{y_{2}}^{\prime}\right)^{\nu_{2}} \cdots\left(\lambda_{y_{m}}^{\prime}\right)^{\nu_{m}} \\
& =\left(\lambda_{x_{1}}^{\prime}\right)^{\varepsilon_{1}} \cdots\left(\lambda_{f x_{n-1}}^{\prime}\right)^{\varepsilon_{n-1}}\left(\lambda_{f x_{n}}^{\prime}\right)^{\varepsilon_{n}}\left(\lambda_{e y_{1}}^{\prime}\right)^{\nu_{1}}\left(\lambda_{e y_{2}}^{\prime}\right)^{\nu_{2}} \cdots\left(\lambda_{y_{m}}^{\prime}\right)^{\nu_{m}} \\
& =(\ldots) \\
& =\left(\lambda_{f x_{1}}^{\prime}\right)^{\varepsilon_{1}} \cdots\left(\lambda_{f x_{n}}^{\prime}\right)^{\varepsilon_{n}}\left(\lambda_{e y_{1}}^{\prime}\right)^{\nu_{1}} \cdots\left(\lambda_{e y_{m}}^{\prime}\right)^{\nu_{m}} \\
& =\bar{\lambda}_{\left[f x_{1}\right]_{e f}^{\varepsilon_{1}}}^{e f} \cdots \bar{\lambda}_{\left[f x_{n}\right]_{e f}^{\varepsilon_{n}}}^{e f} \bar{\lambda}_{\left[e y_{1}\right]_{e f}^{\nu_{1}}}^{e f} \cdots \bar{\lambda}_{\left[e y_{m}\right]_{e f}^{\nu_{m}}}^{e f} \\
& =\lambda_{s t} \text {. }
\end{aligned}
$$

Proposition 3.2.10. The constructed semilattice of groups $N^{\prime}$ is a crossed $S^{\prime}$-module, with $(\lambda, \alpha, \beta), \lambda$ given by Proposition 3.2.9, $\alpha: E\left(S^{\prime}\right) \rightarrow E\left(N^{\prime}\right)$ defined by $\alpha([e])=(\theta(e),[e])$, and $\beta: N^{\prime} \rightarrow S^{\prime}, \beta(a, k)=k$.

Proof: We will prove (CM1) to (CM4). 
(CM1) holds by (3.25).

(CM2) We start with $s=[x]_{e}$ :

$$
\begin{aligned}
\lambda_{[x]_{e}}(\alpha([f])) & =\lambda_{x}^{\prime}(\theta(f),[f]) \\
& =\left(\eta_{x}(\theta(f)) c(x, f, f),[x, f]_{e, f}[x f, f]_{e f, f}[x, f]_{e, f}^{-1}\right) \\
& =\left(\theta\left(x f x^{-1}\right) \theta\left(x x^{-1} f\right),[x, f]_{e, f}[e f][x, f]_{e, f}^{-1}\right) \\
& =(\theta(e f),[e f]) .
\end{aligned}
$$

For $s=[x]_{e}^{-1}$ :

$$
\begin{aligned}
\lambda_{[x]_{e}^{-1}(\alpha([f]))} & =\left(\lambda_{x}^{\prime}\right)^{-1}(\alpha([f])) \\
& =\mu\left(\left[x^{-1}, x\right]_{e}\right)^{-1} \lambda_{x^{-1}}^{\prime}(\alpha([f])) \\
& =\mu\left(\left[x^{-1}, x\right]_{e}\right)^{-1} \alpha\left(\left[x^{-1} f x\right]\right) \\
& =\mu\left(\left[x^{-1}, x\right]_{e}\right)^{-1}\left(\theta\left(x^{-1} f x\right),\left[x^{-1} f x\right]\right) \\
& =\left(\theta\left(x^{-1} f x\right),\left[x^{-1}, x\right]_{e}^{-1}\left[x^{-1} f x\right]\left[x^{-1}, x\right]_{e}\right) \\
& =\left(\theta\left(x^{-1} f x\right),\left[x^{-1} f x\right]\left[x^{-1}, x\right]_{e}^{-1}\left[x^{-1}, x\right]_{e}\right) \\
& =\left(\theta\left(x^{-1} f x\right),\left[x^{-1} f x\right]\left[x^{-1} x\right]_{e}\right) \\
& =\left(\theta\left(x^{-1} f x\right),\left[x^{-1} f x\right]\right) \\
& =\alpha\left(\left[x^{-1} x f\right]\right)=\alpha\left(\left[x x^{-1} f\right]\right) .
\end{aligned}
$$

So, if $s=\left[x_{1}\right]_{e}^{\varepsilon_{1}} \cdots\left[x_{n}\right]_{e}^{\varepsilon_{n}} \in F_{e}$ (this means $x_{i} \in T_{e}$, for all $i=1, \ldots, n$ ), with $\varepsilon_{i} \in\{-1,+1\}$, for $i=1, \ldots, n$, we have:

$$
\begin{aligned}
\lambda_{s}(\alpha([f])) & =\lambda_{\left[x_{1}\right]_{e}^{\varepsilon_{1}}} \cdots \lambda_{\left[x_{n}\right]_{e}^{\varepsilon_{n}}}(\alpha([f])) \\
& =\lambda_{\left[x_{1}\right]_{e}^{\varepsilon_{1}}} \cdots \lambda_{\left[x_{n-1}\right]_{e}^{\varepsilon_{n-1}}}\left(\alpha\left(\left[x_{n} x_{n}^{-1} f\right]\right)\right) \\
& =(\ldots) \\
& =\alpha\left(\left[s s^{-1} f\right]\right) .
\end{aligned}
$$

(CM3) It suffices to take $n=\left(a,[y, z]_{f}\right)$ and $n^{\prime}=\left(b,[v, w]_{g}\right)$, since $\lambda$ is a homomorphism.

$$
\begin{aligned}
\lambda_{\beta(n)}\left(n^{\prime}\right) & =\lambda_{[y, z]_{f}}\left(b,[v, w]_{g}\right) \\
& =\bar{\lambda}_{[y]_{f}}^{f} \bar{\lambda}_{[z]_{f}}^{f} \bar{\lambda}_{[y z]_{f}^{-1}}^{f}\left(b,[v, w]_{g}\right) \\
& =\lambda_{y}^{\prime} \lambda_{z}^{\prime}\left(\lambda_{y z}^{\prime}\right)^{-1}\left(b,[v, w]_{g}\right) \\
& =\mu\left([y, z]_{f}\right) \lambda_{y z}^{\prime}\left(\lambda_{y z}^{\prime}\right)^{-1}\left(b,[v, w]_{g}\right) \\
& =\mu\left([y, z]_{f}\right) \lambda_{f}^{\prime}\left(b,[v, w]_{g}\right) \\
& =\mu\left([y, z]_{f}\right) \alpha([f])\left(b,[v, w]_{g}\right) \\
& =\mu\left([y, z]_{f}\right)\left(\theta(f) b,[f][v, w]_{g}\right) \\
& =\left(\theta(f) b,[y, z]_{f}[f][v, w]_{g}[y, z]_{f}^{-1}\right) \\
& =\left(a a^{-1} b,[y, z]_{f}[v, w]_{g}[y, z]_{f}^{-1}\right)
\end{aligned}
$$




$$
=n n^{\prime} n^{-1} \text {. }
$$

(CM4) Again we take $n=\left(a,[y, z]_{f}\right)$ and start with $s=[x]_{e}$ :

$$
\begin{aligned}
\beta\left(\lambda_{[x]_{e}}\left(a,[y, z]_{f}\right)\right) & =[x, y]_{e, f}[x y, z]_{e f, f}[x, y z]_{e, f}^{-1} \\
& =[x]_{e}[y]_{f}[x y]_{e f}^{-1}[x y]_{e f}[z]_{f}[x y z]_{e f}^{-1}[x y z]_{e f}[y z]_{f}^{-1}[x]_{e}^{-1} \\
& =[x]_{e}[y]_{f}[e f][z]_{f}[e f][y z]_{f}^{-1}[x]_{e}^{-1} \\
& =[x]_{e}[y, z]_{f}[x]_{e}^{-1} \\
& =s \beta(n) s^{-1}
\end{aligned}
$$

Now, for $[x]_{e}^{-1}$ :

$$
\begin{aligned}
\beta\left(\lambda_{[x]_{e}^{-1}}\left(a,[y, z]_{f}\right)\right) & =\beta\left(\left(\lambda_{x}^{\prime}\right)^{-1}\left(a,[y, z]_{f}\right)\right) \\
& =\beta\left(\mu\left(\left[x^{-1}, x\right]_{e}\right)^{-1} \lambda_{x^{-1}}^{\prime}\left(a,[y, z]_{f}\right)\right) \\
& =\beta\left(\mu\left(\left[x^{-1}, x\right]_{e}\right)^{-1}\left(\eta_{x^{-1}}(a)\right) c\left(x^{-1}, y, z\right),\left[x^{-1}, y\right]_{e, f}\left[x^{-1} y, z\right]_{e f, f}\left[x^{-1}, y z\right]_{e, f}^{-1}\right) \\
& =\left[x^{-1}, x\right]_{e}^{-1}\left[x^{-1}, y\right]_{e, f}\left[x^{-1} y, z\right]_{e f, f}\left[x^{-1}, y z\right]_{e, f}^{-1}\left[x^{-1}, x\right]_{e} \\
& =\left[x^{-1} x\right]_{e}[x]_{e}^{-1}\left[x^{-1}\right]_{e}^{-1}\left[x^{-1}\right]_{e}[y]_{f}\left[x^{-1} y\right]_{e f}^{-1}\left[x^{-1} y\right]_{e f}[z]_{f}\left[x^{-1} y z\right]_{e f} \\
& {\left[x^{-1} y z\right]_{e f}^{-1}[y z]_{f}^{-1}\left[x^{-1}\right]_{e}^{-1}\left[x^{-1}\right]_{e}[x]_{e}\left[x^{-1} x\right]_{e}^{-1} } \\
& =[e][x]_{e}^{-1}[y]_{f}[z]_{f}[y z]_{f}^{-1}[x]_{e} \\
& =s \beta(n) s^{-1} .
\end{aligned}
$$

And again, if $s=\left[x_{1}\right]_{e}^{\varepsilon_{1}} \cdots\left[x_{m}\right]_{e}^{\varepsilon_{m}} \in F_{e}$,

$$
\begin{aligned}
\beta\left(\lambda_{s}(n)\right) & =\beta\left(\lambda_{\left[x_{1}\right]_{e}^{\varepsilon_{1}} \ldots\left[x_{m}\right]_{e}^{\varepsilon_{m}}}(n)\right) \\
& =\beta\left(\left(\lambda_{x_{1}}^{\prime}\right)^{\varepsilon_{1}} \cdots\left(\lambda_{x_{m}}^{\prime}\right)^{\varepsilon_{m}}(n)\right) \\
& =\beta\left(\left(\lambda_{x_{1}}^{\prime}\right)^{\varepsilon_{1}} \cdots\left(\lambda_{x_{m-1}}^{\prime}\right)^{\varepsilon_{m-1}}\left(x_{m} \beta(n) x_{m}^{-1}\right)\right. \\
& =(\ldots) \\
& =s \beta(n) s^{-1} .
\end{aligned}
$$

We finally have a sequence

$$
A \stackrel{i}{\rightarrow} N^{\prime} \stackrel{\beta}{\rightarrow} S^{\prime} \stackrel{\pi}{\rightarrow} T
$$

with homomorphisms determined by $i(a)=(a,[e])$, for $a \in A_{e}, \beta\left(a,[x, y]_{e}\right)=[x, y]_{e}$, for $a \in A_{e}$ and a generator $[x, y]_{e}$ of $K_{e}$, and $\pi\left([x]_{e}\right)=x$, for a generator $[x]_{e}$ of $F_{e}$, such that $i(A)=\beta^{-1}\left(E\left(S^{\prime}\right)\right)$ and $\beta\left(N^{\prime}\right)=\pi^{-1}(E(T))$. In order to complete the proof of Theorem 3.2.1, it remains to show that the function $H_{\leqslant}^{3}(T, A) \rightarrow \mathcal{E}(T, A)$ is well-defined.

Proposition 3.2.11. Cohomologous 3-cocycles generate equivalent sequences in $\mathcal{E}(T, A)$.

Proof: We now show that taking two cohomologous (normalized) 3-cocycles the sequences (3.29) determined by each of them are equivalent in the sense of (3.2). 
Taking cohomologous $c, c^{\prime} \in N Z_{\leqslant}^{3}(T, A)$, by Proposition 3.1.19 there exists a normalized 2-cochain $t: T \times$ $T \rightarrow A$ such that $c^{\prime}=\left(\delta^{2} t\right)^{-1} c$.

Constructing the sequences

$$
A \stackrel{i}{\rightarrow} N \stackrel{\beta}{\rightarrow} S \stackrel{\pi}{\rightarrow} T
$$

determined by $c$, and

$$
A \stackrel{i^{\prime}}{\rightarrow} N^{\prime} \stackrel{\beta^{\prime}}{\rightarrow} S^{\prime} \stackrel{\pi^{\prime}}{\rightarrow} T
$$

determined by $c^{\prime}$, define $\varphi_{1}: N \rightarrow N^{\prime}$ by $\varphi_{1}(a,[e]):=(a,[e]), \forall a \in A_{e}, \varphi_{1}\left(1,[y, z]_{f}\right):=\left(t(y, z),[y, z]_{f}\right)$, for $[y, z]_{f}$ generator of $K_{f}$; and $\varphi_{2}=\operatorname{id}_{S}$. Note that both $N=N^{\prime}$ and $S=S^{\prime}$ are the same as semilattices of groups, but the homomorphism $\eta$ of $S$ on $N$ involves $c$, and the homomorphism $\eta^{\prime}$ of $S^{\prime}$ on $N^{\prime}$ involves $c^{\prime}$.

The homomorphism $\varphi_{1}$ is a homomorphism on each group component, since the group $K_{f}$ is freely generated by $[y, z]_{f}$. It is also a homomorphism for the whole semilattice of groups $N$. We will prove

$$
\varphi_{1}\left(\left(a,[y, z]_{f}\right)\left(b,[v, w]_{g}\right)\right)=\varphi_{1}\left(a,[y, z]_{f}\right) \varphi_{1}\left(b,[v, w]_{g}\right) .
$$

Starting with the left-hand side:

$$
\begin{aligned}
\varphi_{1}\left(\left(a,[y, z]_{f}\right)\left(b,[v, w]_{g}\right)\right) & =\varphi_{1}\left(a b,[y, z]_{f}[v, w]_{g}\right) \\
& =\varphi_{1}\left(a b,[g y, g z]_{f g}[f v, f w]_{f g}\right) \\
& =\left(a b t(g y, g z) t(f v, f w),[g y, g z]_{f g}[f v, f w]_{f g}\right) \\
& =\left(a b \theta(g) \theta(f) t(y, z) t(v, w),[y, z]_{f}[v, w]_{g}\right) \\
& =\left(a b t(y, z) t(v, w),[y, z]_{f}[v, w]_{g}\right) \\
& =\left(a t(y, z),[y, z]_{f}\right)\left(b t(v, w),[v, w]_{g}\right),
\end{aligned}
$$

which of course is equal to the right-hand side.

We now show that the diagram (3.2) with these homomorphisms $\varphi_{1}$ and $\varphi_{2}$ commutes:

Since $i: A \rightarrow N$ and $i^{\prime}: A \rightarrow N^{\prime}$ are $i(a)=i^{\prime}(a)=(a,[e])$, for all $a \in A_{e}$, and $\varphi_{1}(a,[e])=(a$, $[e])$, we have $\varphi_{1} \circ i=i^{\prime}$. Analogously to $\pi: S \rightarrow T$ and $\pi^{\prime}: S^{\prime} \rightarrow T$ being $\pi[x]_{e}=\pi^{\prime}[x]_{e}=x$, for all $x \in T$, implies $\pi^{\prime} \circ \varphi_{2}=\pi$.

Now, for the middle square, for $\left(a,[y, z]_{f}\right) \in N_{f}$ :

taking the "right, then down" path: $\varphi_{2} \circ \beta\left(a,[y, z]_{f}\right)=\varphi_{2}[y, z]_{f}$

$$
=[y, z]_{f}
$$

and now taking the "down, then right" path: $\beta^{\prime} \circ \varphi_{1}\left(a,[y, z]_{f}\right)=\beta^{\prime}\left(a t(y, z),[y, z]_{f}\right)$

$$
=[y, z]_{f} \text {. }
$$

Finally, these homomorphisms are compatible with the actions, as in (3.3):

$$
\begin{aligned}
& \varphi_{1}\left(\eta_{[x]_{e}}\left(a,[y, z]_{f}\right)\right) \varphi_{1}\left(\left(\lambda_{x}(a) c(x, y, z),[x, y]_{e, f}[x y, z]_{e f, f}[x, y z]_{e, f}^{-1}\right)\right. \\
& =\left(\lambda_{x}(a) c(x, y, z) t(x, y) t(x y, z) t(x, y z)^{-1},[x, y]_{e, f}[x y, z]_{e f, f}[x, y z]_{e, f}^{-1}\right) \\
& \eta_{\varphi_{2}\left([x]_{e}\right)}^{\prime}\left(\varphi_{1}\left(a,[y, z]_{f}\right)\right)=\eta_{[x]_{e}}^{\prime}\left(a t(y, z),[y, z]_{f}\right)
\end{aligned}
$$




$$
\begin{aligned}
& =\left(\lambda_{x}(a t(y, z)) c^{\prime}(x, y, z),[x, y]_{e, f}[x y, z]_{e f, f}[x, y z]_{e, f}^{-1}\right) \\
& =\left(\lambda_{x}(a) \lambda_{x}(t(y, z)) \lambda_{x}(t(y, z))^{-1} t(x y, z) t(x, y z)^{-1} t(x, y) c(x, y, z),[x, y]_{e, f}[x y, z]_{e f, f}[x, y z]_{e, f}^{-1}\right) \\
& =\left(\lambda_{x}(a) c(x, y, z) t(x, y) t(x y, z) t(x, y z)^{-1},[x, y]_{e, f}[x y, z]_{e f, f}[x, y z]_{e, f}^{-1}\right),
\end{aligned}
$$

We observe that these $\varphi_{1}$ and $\varphi_{2}$ are isomorphisms - the inverse of $\varphi_{1}$ is determined by $\varphi_{1}^{-1}\left([f],[y, z]_{f}\right)=$ $\left(t(y, z)^{-1},[y, z]_{f}\right)$.

Proposition 3.2.11 proves Theorem 3.2.1.

Theorem 3.1.12 defines a function from $\mathcal{E}(T, A)$ to $H_{\leqslant}^{3}(T, A)$. Theorem 3.2.1 defines a function from $H_{\leqslant}^{3}(T, A)$ to $\mathcal{E}(T, A)$, when $T$ is a semilattice of groups. We now show that these functions are inverses to each other.

Theorem 3.2.12. We have a bijective correspondence between $H_{\leqslant}^{3}(T, A)$ and $\mathcal{E}(T, A)$, when $T$ is a semilattice of groups.

Proof: We represent an element of $H_{\leqslant}^{3}(T, A)$ by $c \in N Z_{\leqslant}^{3}(T, A)$, by Proposition 3.1.19.

We now compute the 3-cocycle defined by (3.29) using Proposition 3.1.11.

Define the function $\rho: T \rightarrow S^{\prime}$ as $\rho(x)=[x]_{e}$, for $x \in T_{e}$. This clearly satisfies $\pi \circ \rho=\mathrm{id}_{T}$ and $\rho(e) \rho(x)=$ $[e][x]_{f}=[e x]_{e f}=\rho(e x)$, and since $\pi(\rho(x) \rho(y))=\pi(\rho(x y))$, the function $[\cdot, \cdot]: T \times T \rightarrow \beta(N),[x, y]_{e, f}:=$ $[x]_{e}[y]_{f}[x y]_{e f}^{-1} \in \beta(N)_{\rho(e f)}$ is such that $\rho(x) \rho(y)=[x, y] \rho(x y)$; and we lift this function to $F: T \times T \rightarrow N$ by $F(x, y)=\left(\theta(e f),[x, y]_{e, f}\right)$, which satisfies $\beta(F(x, y))=[x, y]$. We will show that these functions $\rho$ and $F$ together with the cocycle $c$ we started Theorem 3.2.1 satisfy (3.7).

Taking $x \in T_{e}, y \in T_{f}, z \in T_{g}$, the left-hand side of (3.7) is:

$$
\begin{aligned}
\eta_{\rho(x)}(F(y, z)) F(x, y z) & =\lambda_{[x]_{e}}\left(\theta(f g),[y, z]_{f, g}\right)\left(\theta(e f g),[x, y z]_{e, f g}\right) \\
& =\left(\eta_{x}(e) c(x, y, z),[x, y]_{e, f}[x y, z]_{e f, g}[x, y z]_{e, f g}^{-1}\right)\left(\theta(e f g),[x, y z]_{e, f g}\right) \\
& =\left(c(x, y, z),[x, y]_{e, f}[x y, z]_{e f, g}[x, y z]_{e, f g}^{-1}[x, y z]_{e, f g}\right) \\
& =\left(c(x, y, z),[x, y]_{e, f}[x y, z]_{e f, g}[e f g]\right) \\
& =\left(c(x, y, z),[x, y]_{e, f}[x y, z]_{e f, g}\right) ;
\end{aligned}
$$

and the right-hand side of (3.7) is:

$$
\begin{aligned}
i(c(x, y, z)) F(x, y) F(x y, z) & =(c(x, y, z),[e f g])\left(\theta(e f),[x, y]_{e, f}\right)\left(\theta(e f g),[x y, z]_{e f, g}\right) \\
& =\left(c(x, y, z),[x, y]_{e, f}[x y, z]_{e f, g}\right) .
\end{aligned}
$$

And we have the desired equality.

We have shown that the 3-cocycle defined by (3.29) is exactly the 3-cocycle we began with. We can also show that the 3-cocycle determined by a sequence (3.1) generates a sequence (3.29) equivalent to (3.1): it suffices to define $\varphi_{1}: N^{\prime} \rightarrow N$ and $\varphi_{2}: S^{\prime} \rightarrow S$ commuting the diagram and satisfying (3.3).

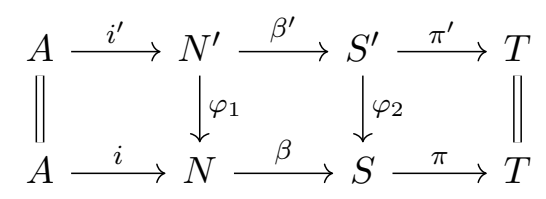


First, define

$$
\begin{aligned}
& \varphi_{1}^{A}: A \rightarrow N \quad \text { and } \quad \varphi_{1}^{K_{f}}: K_{f} \rightarrow N \\
& a \mapsto i(a), \quad[y, z]_{f} \mapsto F(y, z) .
\end{aligned}
$$

Since $K_{f}$ is freely generated by $[y, z]_{f}$, this $\varphi_{1}^{K_{f}}$ is a well-defined homomorphism. We thus have a homomorphism $\varphi_{1}: N_{f}^{\prime} \rightarrow N$ (homomorphism only on each group component) defined by

$$
\varphi\left(a,[y, z]_{f}\right)=i(a) F(y, z)
$$

Now, this $\varphi_{1}$ is a homomorphism (on the whole semilattice of groups):

$$
\begin{aligned}
\varphi_{1}\left(\left(a,[y, z]_{e}\right)\left(b,[v, w]_{f}\right)\right) & =\varphi_{1}\left(a b,[f][y, z]_{e} \cdot[e][v, w]_{f}\right) \\
& =\varphi_{1}^{N_{e f}}\left(a b,[f y, f z]_{e f}[e v, e w]_{e f}\right) \\
& =i(a b) F(f y, f z) F(e v, e w) \\
& =i(a b) \alpha(\rho(f)) F(y, z) \alpha(\rho(e)) F(v, w) \\
& =i(a) i(b) F(y, z) F(v, w) \\
& =i(a) F(y, z) i(b) F(v, w) \\
& =\varphi_{1}\left(a,[y, z]_{e}\right) \varphi_{1}\left(b,[v, w]_{f}\right) .
\end{aligned}
$$

Thus, $\varphi_{1}: N^{\prime} \rightarrow N$ is a homomophism.

Now, define the homomorphism $\varphi_{2}: S^{\prime} \rightarrow S$ by $\varphi[x]_{e}=\rho(x)$.

$$
\begin{aligned}
\varphi_{2}\left([x]_{e}[y]_{f}\right) & =\varphi_{2}\left([f x]_{e f}[e y]_{e f}\right) \\
& =\rho(f x) \rho(e y) \\
& =\rho(f) \rho(x) \rho(e) \rho(y) \\
& =\rho(x) \rho(y) \\
& =\varphi_{2}\left([x]_{e}\right) \varphi_{2}\left([y]_{f}\right) .
\end{aligned}
$$

Taking $a \in A_{e}$, we have

$$
\begin{aligned}
\varphi_{1} \circ i^{\prime}(a) & =\varphi_{1}(a,[e]) \\
& =i(a) F(e, e) \\
& =i(a) \alpha(\rho(e)) \\
& =i(a) ;
\end{aligned}
$$

$$
\begin{aligned}
\pi \circ \varphi_{2}\left([x]_{e}\right) & =\pi(\rho(x)) \\
& =x \\
& =\pi^{\prime}\left([x]_{e}\right) .
\end{aligned}
$$

Now, for $\left(a,[y, z]_{f}\right) \in N_{f}^{\prime}$ :

$$
\begin{aligned}
\beta\left(\varphi_{1}\left(a,[y, z]_{e}\right)\right) & =\beta(i(a) F(y, z)) \\
& =\beta(i(a)) \beta(F(y, z)) \\
& =[e][y, z]_{e} \\
& =[y, z]_{e} ;
\end{aligned}
$$$$
\varphi_{2}\left(\beta^{\prime}\left(a,[y, z]_{e}\right)\right)=\varphi_{2}\left([y, z]_{e}\right)
$$$$
=[y, z]_{e} \text {. }
$$ 
These homomorphisms satisfy (3.3):

$$
\begin{aligned}
\varphi_{1}\left(\lambda_{[x]_{e}}\left(a,[y, z]_{f}\right)\right) & =\varphi_{1}\left(\left(\eta_{x}(a) c(x, y, z),[x, y]_{e, f}[x y, z]_{e f, f}[x, y z]_{e, f}^{-1}\right)\right. \\
= & i\left(\eta_{x}(a) i(c(x, y, z)) F(x, y) F(x y, z) F(x, y z)^{-1}\right. \\
\stackrel{(3.7)}{=} & i\left(\eta_{x}(a)\right) \eta_{\rho(x)}(F(y, z)) \\
= & \eta_{\rho(x)}(i(a) F(y, z)) \\
\lambda_{\varphi_{2}[x]_{e}}\left(\varphi_{1}\left(a,[y, z]_{f}\right)\right) & i\left(\eta_{x}(a)\right) \eta_{\rho(x)}(F(y, z)) .
\end{aligned}
$$




\section{A. Crossed modules of groups}

This appendix is an extended version of Section 5 - Crossed Modules and $H^{3}$ (Sketch), Chapter IV Low-Dimensional Cohomology and Group Extensions from [Bro82].

Let $E$ and $N$ be groups. Suppose we are given an action of $E$ on $N$, denoted $(e, n) \mapsto{ }^{e} n$, as well as a homomorphism $\alpha: N \rightarrow E$ satisfying:

$$
\begin{aligned}
& { }^{\alpha(n)} n^{\prime}=n n^{\prime} n^{-1}, \quad n, n^{\prime} \in N ; \\
& \alpha\left({ }^{e} n\right)=e \alpha(n) e^{-1}, \quad e \in E, n \in N .
\end{aligned}
$$

We then say that $N$ (together with $\alpha$ and the action) is a crossed module over $E$.

Example A.1. (a) Taking $E=$ Aut $N$ and $\alpha(n)$ the inner automorphism associated to $n$. Then (A.1) is true by definition, and for (A.2), taking $\varphi \in$ Aut $N$ :

$$
\begin{aligned}
& \alpha\left({ }^{\varphi} n\right)\left(n^{\prime}\right)=\alpha(\varphi(n))\left(n^{\prime}\right) \quad\left(\varphi \alpha(n) \varphi^{-1}\right)\left(n^{\prime}\right)=\varphi\left(\alpha(n)\left(\varphi^{-1}\left(n^{\prime}\right)\right)\right) \\
& =\varphi(n) n^{\prime} \varphi(n)^{-1} ; \quad=\varphi\left(n \varphi^{-1}\left(n^{\prime}\right) n^{-1}\right) \\
& =\varphi(n) \varphi \circ \varphi^{-1}\left(n^{\prime}\right) \varphi\left(n^{-1}\right) \\
& =\varphi(n) n^{\prime} \varphi(n)^{-1} \text {. }
\end{aligned}
$$

(b) Any abelian $E$-module can be viewed as a crossed module, with $\alpha$ being the trivial map.

(c) Any normal subgroup $N$ is a crossed module with $E$ acting by conjugation and $\alpha$ being the inclusion.

Let $N$ be a crossed module over $E$. We set $A:=\operatorname{ker} \alpha$ and $G:=\operatorname{coker} \alpha$; the latter makes sense because im $\alpha$ is normal in $E$ by (A.2). Note that $A$ is central in $N$, by (A.1), and that the action of $E$ on $N$ induces an action of $G$ on $A$.

We can define the action $g \cdot a:={ }^{e} a$, for $g=[e]$. This does not depend on the choice of representatives: if we also take $g=[f]$, with $f=e \alpha(n)$, then ${ }^{f} a={ }^{e \alpha(n)} a={ }^{e}\left(\operatorname{nan}^{-1}\right)={ }^{e} a$.

Thus, we have a 4-term exact sequence

$$
0 \rightarrow A \stackrel{i}{\rightarrow} N \stackrel{\alpha}{\rightarrow} E \stackrel{\pi}{\rightarrow} G \rightarrow 1,
$$

where $A$ is a $G$-module.

Example A.2. The 4-term exact sequences of Example A.1 are:

(a) $A=\operatorname{ker} \alpha \ni n \Longleftrightarrow \alpha(n)(m)=n m n^{-1}=m, \forall m \in N \Longleftrightarrow n \in C(N)$.

$G=\operatorname{coker} \alpha=\operatorname{Aut} N / \operatorname{Inn} N=\operatorname{Out} N$

$$
0 \rightarrow C(N) \rightarrow N \rightarrow \text { Aut } N \rightarrow \text { Out } N \rightarrow 1 .
$$

(b)

$$
0 \rightarrow E \rightarrow E \rightarrow N \rightarrow N \rightarrow 1
$$

(c)

$$
0 \rightarrow 0 \rightarrow N \rightarrow E \rightarrow E / N \rightarrow 1
$$

It turns out that exact sequences of this form are classified (up to a suitable equivalence relation) by $H^{3}(G, A)$.

More precisely, suppose we start with an arbitrary group $G$ and an arbitrary abelian $G$-module $A$. Consider all possible exact sequences of the form (A.3), where $N$ is a crossed $E$-module such that the action of $E$ on $N$ induces the given 
action of $G$ on $A$. We impose on these exact sequences the smallest equivalence relation such that

$$
0 \rightarrow A \rightarrow N \rightarrow E \rightarrow G \rightarrow 1
$$

is equivalent to

$$
0 \rightarrow A \rightarrow N^{\prime} \rightarrow E^{\prime} \rightarrow G \rightarrow 1
$$

whenever there is a commutative diagram with homomorphisms $\varphi_{1}$ and $\varphi_{2}$ (not necessarily isomorphisms!):

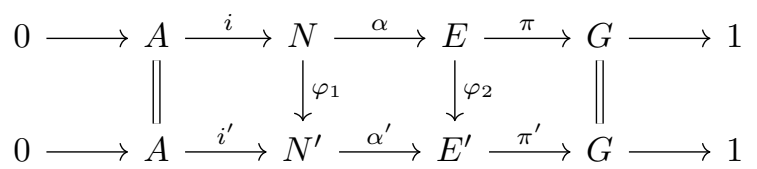

in which the vertical arrows are compatible with the actions of $E$ and $E^{\prime}$ on $N$ and $N^{\prime}$, that is, for $e \in E, n \in N$ :

$$
\varphi_{1}\left({ }^{e} n\right)={ }^{\varphi_{2}(e)} \varphi_{1}(n)
$$

We denote the set of these equivalence classes as $\mathcal{E}(G, A)$.

We then have:

Theorem A.3. There is a 1-1 correspondence between $\mathcal{E}(G, A)$ and $H^{3}(G, A)$.

We will break down the proof in several propositions.

Proposition A.4. Any exact sequence (A.3) determines an element in $H^{3}(G, A)$.

Proof: Given an exact sequence (A.3), choose a function $s: G \rightarrow E$ such that $\pi \circ s=\mathrm{id}_{G}$. Its failure to be multiplicative is measured by a function $f: G \times G \rightarrow \operatorname{ker} \pi$ such that

$$
s(g) s(h)=f(g, h) s(g h) .
$$

Applying $\pi$ to the above, we see that $f$ has codomain $\operatorname{ker} \pi$.

Using the associativity of the product in $E$ :

$$
\begin{array}{rlrl}
s(g)(s(h) s(k)) & =s(g) f(h, k) s(h k) & (s(g) s(h)) s(k)=f(g, h) s(g h) s(k) \\
& =s(g) f(h, k) s(g)^{-1} s(g) s(h k) & & =f(g, h) f(g h, k) s(g h k) \\
& =s(g) f(h, k) f(g, h k) s(g h k)
\end{array}
$$

we get

$$
f(g, h) f(g h, k)={ }^{s(g)} f(h, k) f(g, h k),
$$

where ${ }^{s(g)} f(h, k)=s(g) f(h, k) s(g)^{-1}$.

Since $\operatorname{ker} \pi=\operatorname{im} \alpha$, we can lift $f$ to a function $F: G \times G \rightarrow N$, by $\alpha(F(g, h))=f(g, h)$, and we can ask whether $F$ satisfies an analog of (A.7) (which now should involve the crossed module action of $E$ on $N$ ). The failure of $F$ to do so is measured by a function $c: G \times G \times G \rightarrow A$ such that

$$
{ }^{s(g)} F(h, k) F(g, h k)=i(c(g, h, k)) F(g, h) F(g h, k) .
$$

We now show that $c$ is a 3-cocycle, whose cohomology class is independent of the choices of $F$ and $s$, and this cohomology class is the desired element of $H^{3}(G, A)$. 
To show that $c$ is a 3 -cocycle, we express

$$
{ }^{s(g)}\left[{ }^{s(h)} F(k, l) F(h, k l)\right] F(g, h k l)
$$

in two ways; first computing the bracketed elements:

$$
\begin{aligned}
& s(g)\left[{ }^{s(h)} F(k, l) F(h, k l)\right] F(g, h k l) \\
& \stackrel{(\text { A.8) }}{=} s(g)[i(c(h, k, l) F(h, k) F(h k, l)] F(g, h k l) \\
& ={ }^{s(g)} i(c(h, k, l) \underbrace{s(g)(F(h, k))} \underbrace{s(g)}(F(h k, l)) F(g, h k l) \\
& \stackrel{(\mathrm{A} .8)}{=} s(g) i\left(c ( h , k , l ) i \left(c(g, h, k) F(g, h) F(g h, k) F(g, h k)^{-1} i(c(g, h k, l) F(g, h k) F(g h k, l)\right.\right. \\
& ={ }^{s(g)} i\left(c ( h , k , l ) i \left(c ( g , h , k ) i \left(c(g, h k, l) F(g, h) F(g h, k) F(g, h k)^{-1} F(g, h k) F(g h k, l)\right.\right.\right. \\
& ={ }^{s(g)} i(c(h, k, l) i(c(g, h, k) i(c(g, h k, l) F(g, h) F(g h, k) F(g h k, l) \text {; }
\end{aligned}
$$

second distributing the action of $s(g)$ :

$$
\begin{aligned}
& s(g)\left[{ }^{s(h)} F(k, l) F(h, k l)\right] F(g, h k l) \\
& =s(g)\left({ }^{s(h)}(F(k, l))\right) \underbrace{s(g)(F(h, k l)) F(g, h k l)} \\
& \stackrel{(\text { A.8) }}{=} s(g) s(h) F(k, l) i(c(g, h, k l) F(g, h) F(g h, k l) \\
& =i(c(g, h, k l) \underbrace{\alpha(F(g, h)) s(g h) F(k, l)} F(g, h) F(g h, k l) \\
& \stackrel{(\text { A.1) }}{=} i\left(c(g, h, k l) F(g, h)^{s(g h)} F(k, l) F(g, h)^{-1} F(g, h) F(g h, k l)\right. \\
& =i(c(g, h, k l) F(g, h) \underbrace{s(g h) F(k, l) F(g h, k l)} \\
& \stackrel{(\mathrm{A} .8)}{=} i(c(g, h, k l) F(g, h) i(c(g h, k, l)) F(g h, k) F(g h k, l) \\
& =i(c(g, h, k l) i(c(g h, k, l)) F(g, h) F(g h, k) F(g h k, l) .
\end{aligned}
$$

Cancelling the terms involving $F$, we have

$$
{ }^{s(g)} i(c(h, k, l) i(c(g, h, k) i(c(g, h k, l)=i(c(g, h, k l) i(c(g h, k, l)),
$$

and that means

$$
c \in Z^{3}(G, A) .
$$

- With another choice of lifting for $f: \alpha\left(F^{\prime}(g, h)\right)=f(g, h)$, there exists a function $t: G \times G \rightarrow$ ker $\alpha$ such that $F^{\prime}(g, h)=t(g, h) F(g, h)$.

We have (A.8) for $F^{\prime}$ for a new 3-cocycle $c^{\prime}$ :

$s(g) F^{\prime}(h, k) F^{\prime}(g, h k)=i\left(c^{\prime}(g, h, k)\right) F^{\prime}(g, h) F^{\prime}(g h, k)$.

Since $F^{\prime}=t F:{ }^{s(g)}(t(h, k) F(h, k)) t(g, h k) F(g, h k)=i\left(c^{\prime}(g, h, k)\right) t(g, h) F(g, h) t(g h, k) F(g h, k)$.

${ }^{s(g)} t(h, k){ }^{s(g)} F(h, k) t(g, h k) F(g, h k)=i\left(c^{\prime}(g, h, k)\right) t(g, h) F(g, h) t(g h, k) F(g h, k)$.

$\delta^{2} t(g, h, k) \underbrace{s(g)} F(h, k) F(g, h k)=i\left(c^{\prime}(g, h, k)\right) F(g, h) F(g h, k)$.

Using (A.8) on the bracketed elements:

$\delta^{2} t(g, h, k) i(c(g, h, k)) F(g, h) F(g h, k)=i\left(c^{\prime}(g, h, k)\right) F(g, h) F(g h, k)$.

And, cancelling the terms involving $F: \delta^{2} t(g, h, k) i(c(g, h, k))=i\left(c^{\prime}(g, h, k)\right)$.

Thus, the new 3 -cocycle $c^{\prime}$ is cohomologous to $c$.

- If we choose another function $s^{\prime}: G \rightarrow E$ such that $\pi \circ s^{\prime}=\operatorname{id}_{G}$, there exists a function $r: G \rightarrow \operatorname{ker} \pi=\operatorname{im} \alpha$ such that $s^{\prime}(g)=r(g) s(g)$. For this $s^{\prime}$, we have:

$$
\begin{aligned}
s^{\prime}(g) s^{\prime}(h) & =r(g) s(g) r(h) s(h) \\
& =r(g) s(g) r(h) s(g)^{-1} s(g) s(h) \\
& =r(g)^{s(g)} r(h) f(g, h) s(g h) \\
& =\underbrace{r(g)^{s(g)} r(h) f(g, h) r(g h)^{-1}} s^{\prime}(g h)
\end{aligned}
$$


and we can choose the bracketed expression as $f^{\prime}$ and write:

$$
f^{\prime}(g, h) r(g h)=r(g)^{s(g)} r(h) f(g, h) .
$$

We can also write this as: $f^{\prime}(g, h) r(g h)=r(g)^{s(g)} r(h) f(g, h)$

$$
\begin{aligned}
& =r(g) s(g) r(h) s(g)^{-1} f(g, h) \\
& =s^{\prime}(g) r(h) s^{\prime}(g)^{-1} r(g) f(g, h) \\
& =s^{\prime}(g) r(h) r(g) f(g, h) .
\end{aligned}
$$

So we have:

$$
\begin{aligned}
f^{\prime}(g, h) r(g h) & =r(g)^{s(g)} r(h) f(g, h) \\
& ={ }^{s^{\prime}(g)} r(h) r(g) f(g, h) .
\end{aligned}
$$

Again, we can lift this $f^{\prime}$ to a function $F^{\prime}: G \times G \rightarrow N$ such that $\alpha\left(F^{\prime}(g, h)\right)=f^{\prime}(g, h)$.

Now, since $r: G \rightarrow \operatorname{im} \alpha$, there exists a function $R: G \rightarrow N$ such that $\alpha(R(g))=r(g)$, and (A.9b) becomes:

$$
\begin{aligned}
& \alpha\left(F^{\prime}(g, h)\right) \alpha(R(g h))=s^{\prime}(g) \alpha(R(h)) s^{\prime}(g)^{-1} \alpha(R(g)) \alpha(F(g, h)) \\
& \stackrel{(\text { A.2) }}{=} \alpha\left(s^{\prime}(g) R(h)\right) \alpha(R(g)) \alpha(F(g, h))
\end{aligned}
$$

and this implies that there exists a function $w: G \times G \rightarrow \operatorname{ker} \alpha=A$ such that

$$
F^{\prime}(g, h) R(g h)=w(g, h)^{s^{\prime}(g)} R(h) R(g) F(g, h) .
$$

$$
\begin{aligned}
& s^{\prime}(g) F^{\prime}(h, k) \underbrace{F^{\prime}(g, h k) R(g h k)}=s^{\prime}(g) F^{\prime}(h, k) w(g, h k)^{s^{\prime}(g)} R(h k) R(g) F(g, h k) \\
& =w(g, h k)^{s^{\prime}(g)}(\underbrace{F^{\prime}(h, k) R(h k)}) R(g) F(g, h k) \\
& =w(g, h k) s^{s^{\prime}(g)}\left(w(h, k) s^{s^{\prime}(h)} R(k) R(h) F(h, k)\right) R(g) F(g, h k) \\
& =w(g, h k)^{s^{\prime}(g)} w(h, k) s^{\prime}(g) s^{\prime}(h) R(k) s^{\prime}(g) R(h) \underbrace{s^{\prime}(g)} F(h, k) R(g) F(g, h k) \\
& =w(g, h k)^{s^{\prime}(g)} w(h, k) s^{s^{\prime}(g) s^{\prime}(h)} R(k)^{s^{\prime}(g)} R(h)^{\alpha(R(g)) s(g)} F(h, k) R(g) F(g, h k) \\
& =w(g, h k)^{s^{\prime}(g)} w(h, k) s^{\prime}(g) s^{\prime}(h) R(k) s^{\prime}(g) R(h) R(g){ }^{s(g)} F(h, k) R(g)^{-1} R(g) F(g, h k) \\
& =w(g, h k) s^{\prime}(g) w(h, k) s^{\prime}(g) s^{\prime}(h) R(k) s^{s^{\prime}(g)} R(h) R(g) \underbrace{s(g)} F(h, k) F(g, h k) \\
& =w(g, h k)^{s^{\prime}(g)} w(h, k) s^{\prime}(g) s^{\prime}(h) R(k) s^{\prime}(g) R(h) R(g) i(c(g, h, k)) F(g, h) F(g h, k) \\
& =i(c(g, h, k)) w(g, h k) s^{s^{\prime}(g)} w(h, k) s^{s^{\prime}(g) s^{\prime}(h)} R(k) \underbrace{s^{\prime}(g)} R(h) R(g) F(g, h) F(g h, k) \\
& =i(c(g, h, k)) w(g, h k)^{s^{\prime}(g)} w(h, k) \underbrace{s^{\prime}(g) s^{\prime}(h)} R(k) \underbrace{w(g, h)^{-1} F^{\prime}(g, h) R(g h) F(g h, k)} \\
& =i(c(g, h, k)) w(g, h k) s^{\prime}(g) w(h, k) w(g, h)^{-1 \alpha\left(F^{\prime}(g, h)\right) s^{\prime}(g h)} R(k) F^{\prime}(g, h) R(g h) F(g h, k) \\
& =i(c(g, h, k)) w(g, h k)^{s^{\prime}(g)} w(h, k) w(g, h)^{-1} F^{\prime}(g, h) s^{\prime}(g h) R(k) F^{\prime}(g, h)^{-1} F^{\prime}(g, h) R(g h) F(g h, k) \\
& =i(c(g, h, k)) w(g, h k)^{s^{\prime}(g)} w(h, k) w(g, h)^{-1} F^{\prime}(g, h) \underbrace{s^{\prime}(g h)} R(k) R(g h) F(g h, k) \\
& =i(c(g, h, k)) w(g, h k)^{s^{\prime}(g)} w(h, k) w(g, h)^{-1} F^{\prime}(g, h) w(g h, k)^{-1} F^{\prime}(g h, k) R(g h k) \\
& =i(c(g, h, k)) w(g, h k)^{s^{\prime}(g)} w(h, k) w(g, h)^{-1} w(g h, k)^{-1} F^{\prime}(g, h) F^{\prime}(g h, k) R(g h k)
\end{aligned}
$$

Cancelling the term $R(g h k)$, we have

$$
\begin{gathered}
{ }^{s^{\prime}(g)} F^{\prime}(h, k) F^{\prime}(g, h k)=i(c(g, h, k)) w(g, h k)^{s^{\prime}(g)} w(h, k) w(g, h)^{-1} w(g h, k)^{-1} F^{\prime}(g, h) F^{\prime}(g h, k) \\
\Longrightarrow i\left(c^{\prime}(g, h, k)\right)=i(c(g, h, k)) \delta^{2} w(g, h, k) .
\end{gathered}
$$

Thus, we have shown that given a 4-term exact sequence like (A.3) we obtain a 3-cocycle whose cohomology class is independent of the possible choices.

For the next step, we need a lemma: 
Lemma A.5. [EM47, Lemmas 6.1 and 6.2] Every cocycle is cohomologous to a normalized cocycle. Every normalized coboundary is the coboundary of a normalized cochain.

Proposition A.6. Any element in $H^{3}(G, A)$ determines a sequence like (A.3).

Proof: By Lemma A.5, we can take a normalized 3-cocycle $c$ to represent an element in $H^{3}(G, A)$, that is $c \in N Z^{3}(G, A)$. Next, we construct a sequence like (A.3) (using [Mac49] as reference).

Given the group $G$, we define the free group $E^{\prime}$ generated by the symbols $[g]$, for all $g \in G, g \neq 1$. For this group, we have $\pi: E^{\prime} \rightarrow G$ an epimorphism determined by $\pi[g]=g$. The group $K:=\operatorname{ker} \pi$ is also free (since it is a subgroup of a free group), with generators $[g, h]:=[g][h][g h]^{-1}$, for $g, h \in G, g \neq 1, h \neq 1$.

We define the direct product $N^{\prime}:=A \times K$ and a function $r(g, h)= \begin{cases}{[g, h]} & g \neq 1 \neq h, \\ 1 & \text { otherwise. }\end{cases}$

For each $g \in G$, we define $\eta_{g} \in$ Aut $N^{\prime}$ by:

$$
\eta_{g}(a):=g \cdot a, \forall a \in A ; \quad \eta_{g}[h, k]:=c(g, h, k) r(g, h) r(g h, k) r(g, h k)^{-1},[h, k] \in K
$$

Since the 3-cocycle $c$ was taken normalized, this last relation also holds for $h=1$ or $k=1$ :

For $h=1: \eta_{g}(1)=c(g, 1, k) r(g, 1) r(g 1, k) r(g, 1 k)^{-1} \Longrightarrow \eta_{g}(1)=1$, and likewise for $k=1$. So this last relation is

$$
\eta_{g} r(h, k):=c(g, h, k) r(g, h) r(g h, k) r(g, h k)^{-1}
$$

CLAIM: $\eta_{g} \eta_{h}=\mu(r(g, h)) \eta_{g h}$, with $\mu(r(g, h))$ is the conjugation by $r(g, h)$.

First, for $a \in A$, since each $\eta$ is the action of $G$ on $A$, we have the needed equality.

So, it suffices to show that the endomorphism on each side has the same effect on any generator $[k, l]$ of $K$.

$$
\begin{aligned}
\eta_{g} \eta_{h} & {[k, l]=\eta_{g}\left[c(h, k, l) r(h, k) r(h k, l) r(h, k l)^{-1}\right] } \\
= & g \cdot c(h, k, l) c(g, h, k) r(g, h) r(g h, k) r(g, h k)^{-1} c(g, h k, l) r(g, h k) r(g h k, l) r(g, h k l)^{-1} \\
& r(g, h k l) r(g h, k l)^{-1} r(g, h)^{-1} c(g, h, k l)^{-1} .
\end{aligned}
$$

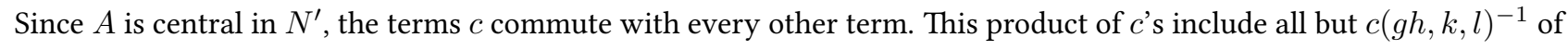
$\delta^{3} c(g, h, k, l)=1$. This results in:

$$
\begin{aligned}
& \eta_{g} \eta_{h}[k, l]=r(g, h)(g \cdot c(h, k, l)) c(g, h, k) c(g, h k, l) c(g, h, k l)^{-1} r(g h, k) r(g h k, l) r(g h, k l)^{-1} r(g, h)^{-1} \\
& \quad=r(g, h) c(g h, k, l) r(g h k, l) r(g h, k l)^{-1} r(g, h)^{-1} \\
& \quad=r(g, h) \eta_{g h} r(k, l) r(g, h)^{-1} \\
& \quad=\mu(r(g, h)) \eta_{g h}[k, l] .
\end{aligned}
$$

Now, since the product $\eta_{g} \eta_{g^{-1}}=\mu\left(r\left(g, g^{-1}\right)\right) \eta_{1}=\mu\left(r\left(g, g^{-1}\right)\right)$ is an (inner) automorphism of $N^{\prime}$, we have that $\eta_{g}$ is an epimorphism of $N^{\prime}$ and $\eta_{g^{-1}}$ is a monomorphism of $N^{\prime}$. Since $g \in G$ is arbitrary, we then have that each $\eta_{g}$ is an automorphism of $N^{\prime}$. It follows that $\eta$ induces a homomorphism $\eta: E^{\prime} \rightarrow \operatorname{Aut} N^{\prime}$

$$
\eta_{[g]}(a,[h, k]):=\left((g \cdot a) c(g, h, k), r(g, h) r(g h, k) r(g, h k)^{-1}\right) .
$$

And we have an exact sequence

$$
0 \rightarrow A \stackrel{i}{\rightarrow} N^{\prime} \stackrel{\alpha}{\rightarrow} E^{\prime} \stackrel{\pi}{\rightarrow} G \rightarrow 1
$$

with homomorphisms determined by $i(a)=(a, 1)$, for $a \in A, \alpha(a,[g, h])=[g, h]$, for $a \in A$, and a generator $[g, h]$ of $K$, and $\pi[g]=g$, for a generator $[g]$ of $E$, satisfying (A.1) and (A.2). 
For (A.1): taking $(a,[g, h]) \in N^{\prime}$, we have $\alpha(a,[g, h])=[g, h]$ and

$\eta_{[g, h]}=\eta_{[g][h][g h]^{-1}}=\eta_{[g]} \eta_{[h]} \eta_{[g h]}^{-1}=\mu([g, h]) \eta_{[g h]} \eta_{[g h]}^{-1}=\mu([g, h])$, and we have (A.1) for any $a \in A$ and a generator $[g, h] \in K$.

For (A.2): taking $[g] \in E^{\prime},(a,[h, k]) \in N^{\prime}$, if $g h \neq 1$ and $h k \neq 1$ :

$$
\begin{aligned}
& \alpha \\
& \left.\quad \eta_{[g]}(a,[h, k])\right)=\alpha\left((g \cdot a) c(g, h, k),[g, h][g h, k][g, h k]^{-1}\right) \\
& \quad=[g, h][g h, k][g, h k]^{-1} \\
& \quad=[g][h][g h]^{-1}[g h][k][g h k]^{-1}[g h k][h k]^{-1}[g]^{-1} \\
& \quad=[g][h][k][h k]^{-1}[g]^{-1} \\
& \quad=[g][h, k][g]^{-1} \\
& \quad=[g] \alpha(a,[h, k])[g]^{-1}
\end{aligned}
$$

If $g h=1$ and $h k \neq 1$ :

$$
\begin{aligned}
\alpha & \left(\eta_{[g]}(a,[h, k])\right)=\alpha\left((g \cdot a) c(g, h, k),[g, h][g, h k]^{-1}\right) \\
& =[g, h][g, h k]^{-1} \\
& =[g][h][g h]^{-1}[g h k][h k]^{-1}[g]^{-1} \\
& =[g][h][k][h k]^{-1}[g]^{-1} \\
& =[g][h, k][g]^{-1} \\
& =[g] \alpha(a,[h, k])[g]^{-1} .
\end{aligned}
$$

If $g h \neq 1$ and $h k=1$ :

$$
\begin{aligned}
\alpha & \left(\eta_{[g]}(a,[h, k])\right)=\alpha((g \cdot a) c(g, h, k),[g, h][g h, k]) \\
& =[g, h][g h, k] \\
& =[g][h][g h]^{-1}[g h][k][g]^{-1} \\
& =[g][h][k][g]^{-1} \\
& =[g][h, k][g]^{-1} \\
& =[g] \alpha(a,[h, k])[g]^{-1} .
\end{aligned}
$$

With Propositions A.4 and A.6 we defined two functions: one from $\mathcal{E}(G, A)$ to $H^{3}(G, A)$ and one from $H^{3}(G, A)$ to $\mathcal{E}(G, A)$. We now show that these functions are inverses to each other.

Proposition A.7. There is a one-to-one correspondence between $H^{3}(G, A)$ and $\mathcal{E}(G, A)$.

Proof: We first compute the 3-cocycle defined by (A.10) using Proposition A.4.

We define the function $s: G \rightarrow E^{\prime}$ as $s(g)=[g], \forall g \neq 1$ and $s(1)=1$, satisfying $\pi \circ s=\mathrm{id}_{G}$. Its failure to be multiplicative is measured by the function $f: G \times G \rightarrow \operatorname{ker} \pi, f(g, h)=s(g) s(h) s(g h)^{-1}=[g][h][g h]^{-1}=[g, h]$, and we see that, since $s(1)=1$, we can define $f: G \times G \rightarrow \operatorname{ker} \pi$ to be $f(g, h)=r(g, h)$; we lift it to $F: G \times G \rightarrow N$ by $F(g, h)=(1, r(g, h))$. We will show that these functions $s$ and $F$ together with the cocycle $c$ we started Proposition A.6 satisfy (A.8).

The left-hand side of (A.8) is:

$$
\begin{aligned}
{ }^{s(g)} F(h, k) F(g, h k) & =\eta_{[g]}(1, r(h, k))(1, r(g, h k)) \\
& =\left((g \cdot 1) c(g, h, k), r(g, h) r(g h, k) r(g, h k)^{-1}\right)(1, r(g, h k)) \\
& =\left(c(g, h, k), r(g, h) r(g h, k) r(g, h k)^{-1} r(g, h k)\right) \\
& =(c(g, h, k), r(g, h) r(g h, k)) ;
\end{aligned}
$$

and the right-hand side of (A.8) is: 


$$
\begin{aligned}
i(c(g, h, k)) F(g, h) F(g h, k) & =(c(g, h, k), 1)(1, r(g, h))(1, r(g h, k)) \\
& =(c(g, h, k), r(g, h) r(g h, k)) .
\end{aligned}
$$

And we have the desired equality.

We have shown that the 3 -cocycle defined by (A.10) is exactly the 3 -cocycle we began with. With this, we can also show that the 3-cocycle determined by a sequence (A.3) generates a sequence (A.10) equivalent to (A.10): it suffices to define $\varphi_{1}: N^{\prime} \rightarrow N$ by $\varphi_{1}(a, 1):=i(a), \varphi_{1}(1,[g, h]):=F(g, h), \varphi_{2}: E^{\prime} \rightarrow E$ by $\varphi_{2}[g]=s(g)$.

These homomorphisms satisfy (A.5):

$$
\begin{aligned}
\varphi_{1}\left(\eta_{[g]}(a,[h, k])\right) & =\varphi_{1}\left((g \cdot a) c(g, h, k), r(g, h) r(g h, k) r(g, h k)^{-1}\right) \\
& =i(g \cdot a) i(c(g, h, k)) F(g, h) F(g h, k) F(g, h k)^{-1} \\
& \stackrel{(\text { A.8) }}{=} i(g \cdot a)^{s(g)} F(h, k) \\
\varphi_{2}[g] & \varphi_{1}(a,[h, k])= \\
= & s(g)(i(a) F(h, k)) \\
= & i(g \cdot a)^{s(g)} F(h, k) .
\end{aligned}
$$

Proposition A.8. Equivalent elements of $\mathcal{E}(G, A)$ generate the same 3 -cocycle in $H^{3}(G, A)$.

Proof: Starting with equivalent exact sequences

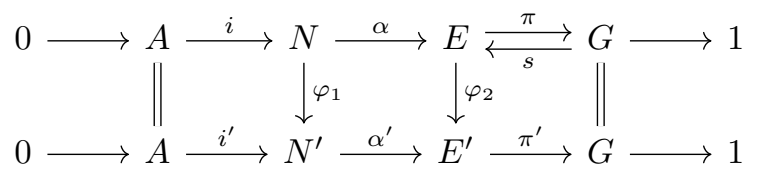

we do the whole procedure from Proposition A.4 to find the 3-cocycle determined by the above sequence, obtaining $s: G \rightarrow E, f: G \times G \rightarrow \operatorname{ker} \pi, F: G \times G \rightarrow N$, and $c: G \times G \times G \rightarrow A$, satisfying (A.1) and (A.2). And since the cohomology class of the cocycle determined by the sequence is independent of the choices, we choose $s^{\prime}: G \rightarrow E^{\prime}$ to be $s^{\prime}:=\varphi_{2} \circ s$.

$$
\begin{aligned}
\pi \circ s=\operatorname{id}_{G} & \Longrightarrow \pi^{\prime} \circ \varphi_{2} \circ s=\operatorname{id}_{G} \\
s^{\prime}(g) s^{\prime}(h) & =\varphi_{2}(s(g)) \varphi_{2}(s(h)) \\
& =\varphi_{2}(f(g, h) s(g h)) \\
& =\varphi_{2}(f(g, h)) \varphi_{2}(s(g h)) \\
& =\varphi_{2}(f(g, h)) s^{\prime}(g h)
\end{aligned}
$$

We choose $f^{\prime}(g, h):=\varphi_{2}(f(g, h))$ to be the measure of the failure of $s$ to be multiplicative.

Applying $\varphi_{1}$ to (A.8) for the above sequence: $\varphi_{1}\left({ }^{s(g)} F(h, k) F(g, h k)\right)=\varphi_{1}(i(c(g, h, k)) F(g, h) F(g h, k))$.

Using (A.5) on the left-hand side: $\varphi_{1}\left({ }^{s(g)} F(h, k)\right) \varphi_{1}(F(g, h k))=\varphi_{2}(s(g)) \varphi_{1}(F(h, k)) \varphi_{1}(F(g, h k))$.

On the right-hand side, we use the commutativity of the diagram:

$\varphi_{1}(i(c(g, h, k))) \varphi_{1}(F(g, h)) \varphi_{1}(F(g h, k))=i^{\prime}(c(g, h, k)) \varphi_{1}(F(g, h)) \varphi_{1}(F(g h, k))$.

So we choose $F^{\prime}(g, h):=\varphi_{1}(F(g, h))$, which in fact lifts $f^{\prime}$ to $N: \alpha^{\prime}\left(F^{\prime}(g, h)\right)=\alpha^{\prime}\left(\varphi_{1}(F(g, h))\right)$

$$
\begin{aligned}
& =\varphi_{2}(\alpha(F(g, h))) \\
& =\varphi_{2}(f(g, h)) \\
& =f^{\prime}(g, h) .
\end{aligned}
$$

Therefore, equivalent sequences determine the same 3-cocycle $c$.

Proposition A.9. Cohomologous 3-cocycles generate equivalent sequences in $\mathcal{E}(G, A)$. 
Proof: We now show that taking two cohomologous (normalized) 3-cocycles the sequences (A.10) determined by each of them are equivalent in the sense of (A.4).

Taking $c, c^{\prime} \in N Z^{3}(G, A)$ cohomologous, there exists a normalized 2-cochain $t: G \times G \rightarrow A$ by Lemma A.5 such that $c^{\prime}=\left(\delta^{2} t\right)^{-1} c$

Constructing the sequences $0 \rightarrow A \stackrel{i}{\rightarrow} N \stackrel{\alpha}{\rightarrow} E \stackrel{\pi}{\rightarrow} G \rightarrow 1$ determined by $c$, and $0 \rightarrow A \stackrel{i^{\prime}}{\rightarrow} N^{\prime} \stackrel{\alpha^{\prime}}{\rightarrow} E^{\prime} \stackrel{\pi^{\prime}}{\rightarrow} G \rightarrow 1$ determined by $c^{\prime}$, define $\varphi_{1}: N \rightarrow N^{\prime}$ by $\varphi_{1}(a, 1):=(a, 1), \forall a \in A, \varphi_{1}(1,[g, h]):=(t(g, h),[g, h])$, for $[g, h]$ generator of $K$; and $\varphi_{2}=\operatorname{id}_{E}$. Note that both $N=N^{\prime}$ and $E=E^{\prime}$ are the same as groups, but the action of $N$ on $E$ involves $c$, and the action of $N^{\prime}$ on $E^{\prime}$ involves $c^{\prime}$.

We now show that the diagram (A.4) with these homomorphisms $\varphi_{1}$ and $\varphi_{2}$ commutes:

Since $i: A \rightarrow N$ and $i^{\prime}: A \rightarrow N^{\prime}$ are $i(a)=i^{\prime}(a)=(a, 1), \forall a \in A$, and $\varphi_{1}(a, 1)=(a, 1)$, we have $\varphi_{1} \circ i=i^{\prime}$. Analogously to $\pi: E \rightarrow G$ and $\pi^{\prime}: E^{\prime} \rightarrow G$ being $\pi[g]=\pi^{\prime}[g]=g, \forall g \in G$, implies $\pi^{\prime} \circ \varphi_{2}=\pi$.

Now, for the middle square, for $(a,[g, h]) \in N$ :

taking the "right, then down" path: $\varphi_{2} \circ \alpha(a,[g, h])=\varphi_{2}[g, h]$

$$
=[g, h]
$$

and now taking the "down, then right" path: $\alpha^{\prime} \circ \varphi_{1}(a,[g, h])=\alpha^{\prime}(a t(g, h),[g, h])$

$$
=[g, h] \text {. }
$$

Finally, these homomorphisms are compatible with the actions, as in (A.5):

$$
\begin{gathered}
\varphi_{1}\left(\eta_{[g]}(a,[h, k])\right) \varphi_{1}\left((g \cdot a) c(g, h, k), r(g, h) r(g h, k) r(g, h k)^{-1}\right) \\
\left.=(g \cdot a) c(g, h, k) t(g, h) t(g h, k) t(g, h k)^{-1}, r(g, h) r(g h, k) r(g, h k)^{-1}\right) ; \\
\eta_{\varphi_{2}[g]}^{\prime}\left(\varphi_{1}(a,[h, k])\right)=\eta_{[g]}^{\prime}(a t(h, k),[h, k]) \\
=\left(g \cdot(a t(h, k)) c^{\prime}(g, h, k), r(g, h) r(g h, k) r(g, h k)^{-1}\right) \\
=\left((g \cdot a)(g \cdot t(h, k))(g \cdot t(h, k))^{-1} t(g h, k) t(g, h k)^{-1} t(g, h) c(g, h, k), r(g, h) r(g h, k) r(g, h k)^{-1}\right) \\
=\left((g \cdot a) t(g h, k) t(g, h k)^{-1} t(g, h) c(g, h, k), r(g, h) r(g h, k) r(g, h k)^{-1}\right) .
\end{gathered}
$$

We observe that these $\varphi_{1}$ and $\varphi_{2}$ are isomorphisms - the inverse of $\varphi_{1}$ is determined by $(1,[g, h]) \mapsto\left(t(g, h)^{-1},[g, h]\right)$. 


\section{Bibliography}

[AAR17] E. R. Alvares, M. M. Alves, and M. J. Redondo, Cohomology of partial smash products, Journal of Algebra 482 (2017), 204-223.

[AS06] K. Auinger and M. B. Szendrei, On F-inverse covers of inverse monoids, Journal of Pure and Applied Algebra 204 (2006), no. 3, 493 - 506.

[Bro82] K. S. Brown, Cohomology of Groups, Graduate Texts in Mathematics, Springer, 1982.

[CP61] A. H. Clifford and G. B. Preston, The Algebraic Theory of Semigroups, vol. 1, Mathematical Surveys and Monographs, American Mathematical Society, 1961.

[CP67] _ The Algebraic Theory of Semigroups, vol. 2, American Mathematical Society, 1967.

[DE05] M. Dokuchaev and R. Exel, Associativity of Crossed Products by Partial Actions, Enveloping Actions and Partial Representations, Transactions of the American Mathematical Society 357 (2005), no. 5 , 1931-1952.

[DES08] M. Dokuchaev, R. Exel, and J.J. Simón, Crossed products by twisted partial actions and graded algebras, Journal of Algebra 320 (2008), no. 8, 3278-3310.

[DK15] M. Dokuchaev and M. Khrypchenko, Partial cohomology of groups, Journal of Algebra 427 (2015), $142-182$

[DK17] _ _ Twisted partial actions and extensions of semilattices of groups by groups, International Journal of Algebra and Computation 27 (2017), no. 07, 887-933.

[DK18] _ Partial cohomology of groups and extensions of semilattices of abelian groups, Journal of Pure and Applied Algebra 222 (2018), no. 10, 2897-2930.

[DN10] M. Dokuchaev and B. Novikov, Partial projective representations and partial actions, Journal of Pure and Applied Algebra 214 (2010), no. 3, 251-268.

[Dok19] M. Dokuchaev, Recent developments around partial actions, São Paulo J. Math. Sci. 13 (2019), 195-247.

[EM47] S. Eilenberg and S. MacLane, Cohomology Theory in Abstract Groups. I, Annals of Mathematics 48 (1947), no. 1, 51-78.

[Exe98] R. Exel, Partial actions of groups and actions of inverse semigroups, Proceedings of the American Mathematical Society 126 (1998), no. 12, 3481-3494.

[Exe17] _ Partial dynamical systems, fell bundles and applications, Mathematical Surveys and Monographs, American Mathematical Society, 2017.

[KL04] J. Kellendonk and M. V. Lawson, Partial actions of groups, International Journal of Algebra and Computation 14 (2004), no. 01, 87-114.

[Lau75] H. Lausch, Cohomology of inverse semigroups, Journal of Algebra 35 (1975), no. 1-3, 273-303. 
[Law98] M. V. Lawson, Inverse Semigroups: The Theory of Partial Symmetries, World Scientific, 1998.

[Mac49] S. MacLane, Cohomology Theory in Abstract Groups. III: Operator Homomorphisms of Kernels, Annals of Mathematics 50 (1949), no. 3, 736-761.

[Mac94] _ Homology, Grundlehren der mathematischen Wissenschaften, Springer Berlin Heidelberg, 1994.

[MR77] D. B. McAlister and N. R. Reilly, E-unitary covers for inverse semigroups, Pacific J. Math. 68 (1977), no. $1,161-174$

[Pet84] M. Petrich, Inverse semigroups, Pure and applied mathematics, Wiley, 1984.

[PR79] M. Petrich and N. R. Reilly, A representation of E-unitary inverse semigroups, The Quarterly Journal of Mathematics 30 (1979), no. 3, 339-350.

[Sze89] M. B. Szendrei, A note on Birget-Rhodes expansion of groups, Journal of Pure and Applied Algebra 58 (1989), no. 1, 93-99. 